\title{
A Multifaceted Performance Model for the Multiple Percussion Performance Practice: Performance Analysis of Select Works toward Developing a Graduate Curriculum
}

\author{
Mitchell Joseph Greco \\ West Virginia University, mjgreco@mix.wvu.edu
}

Follow this and additional works at: https://researchrepository.wvu.edu/etd

Part of the Music Pedagogy Commons

\section{Recommended Citation \\ Greco, Mitchell Joseph, "A Multifaceted Performance Model for the Multiple Percussion Performance Practice: Performance Analysis of Select Works toward Developing a Graduate Curriculum" (2020). Graduate Theses, Dissertations, and Problem Reports. 7564. \\ https://researchrepository.wvu.edu/etd/7564}

This Dissertation is protected by copyright and/or related rights. It has been brought to you by the The Research Repository @ WVU with permission from the rights-holder(s). You are free to use this Dissertation in any way that is permitted by the copyright and related rights legislation that applies to your use. For other uses you must obtain permission from the rights-holder(s) directly, unless additional rights are indicated by a Creative Commons license in the record and/ or on the work itself. This Dissertation has been accepted for inclusion in WVU Graduate Theses, Dissertations, and Problem Reports collection by an authorized administrator of The Research Repository @ WVU. For more information, please contact researchrepository@mail.wvu.edu. 
A Multifaceted Performance Model for the Multiple Percussion Performance Practice: Performance Analysis of Select Works toward Developing a Graduate Curriculum

Mitchell Joseph Greco 
A Multifaceted Performance Model for the Multiple Percussion Performance Practice:

Performance Analysis of Select Works toward Developing a Graduate Curriculum

Mitchell J. Greco

Dissertation submitted to

the College of Creative Arts

at West Virginia University

in partial fulfillment of the requirements for the degree of

Doctor of Musical Arts

in Music Performance

Michael Vercelli, DMA, Chair

Mitchell Arnold, DM

Yoav Kaddar, $\mathrm{PhD}$

Travis Stimeling, $\mathrm{PhD}$

George Willis, MM

School of Music

Morgantown, West Virginia

2020

Keywords: Multiple percussion, curriculum

Copyright (@2020 by Mitchell J. Greco

All rights reserved 


\begin{abstract}
A Multifaceted Performance Model for the Multiple Percussion Performance Practice: Performance Analysis of Select Works toward Developing a Graduate Curriculum

Mitchell J. Greco
\end{abstract}

The purpose of this document is to articulate the various dimensions of the solo multiple percussion performance practice, the primary objective being to construct appropriate graduate curricula. It was found that the necessary skills and concepts could be categorized into four dimensions that make up the multi-faceted model: (1) equipment and setup, (2) notational style, (3) technical demands, and (4) conceptual performance. Requisite skills were posited from performance analyses of over fifty solo works that range from intermediate to advanced difficulty. Representative works are described that might effectively introduce and challenge these requisite skills and concepts, as are exemplary pieces that require their mastery. One piece that I performed for recital within my doctoral program was selected for each dimension to provide experiential details: The Anvil Chorus is used to describe equipment and setup, Psappha for notational style and devices, Thirteen Drums for technical facility, and Tunnels for the performance concept. I conclude the document with sample program curricula suggested for master's and doctoral level, as well as curricula specific to each dimension of the performance model. It is my hope that the percussion instructor will use this model to analyze their own inventory and to include future additions to the literature for creating an effective and comprehensive graduate multiple percussion curriculum. 


\section{ACKNOWLEDGEMENTS}

This document could not have been completed without the assistance, guidance and support of many people. I would firstly like to thank my family for their constant support and encouragement. My fiancé, Caryn, deserves special attention as she continued to stand by my side as we traversed our doctoral programs together.

I would like to thank my research advisor, Dr. Michael Vercelli, for his guidance throughout the research and writing of this document. I would like to thank the graduate committee members for their valuable contributions to my doctoral program as well: Prof. George Willis, Dr. Travis Stimeling, Dr. Mitchell Arnold and Dr. Yoav Kaddar. I would like to thank Prof. Paul Scea and Dr. Janet Robbins, who previously served on the committee before their retirements. A special thank you to Molly Reinhoudt, who painstakingly copyedited the document.

I would like to thank all my prior teachers. Dr. Kay Stonefelt and Dr. Rob Falvo, who gave me a solid foundation in music and percussion while modeling how to be a good mentor and person. Dr. Kazadi wa Mukuna, Dr. Laurie Semmes and the late Dr. Halim El-Dabh, for encouraging my interest in scholarly inquiry while challenging - and ultimately expanding - my research and writing abilities. Last, but certainly not least, a special acknowledgement to the great Bernard Woma, whose spirit I attempt to summon every time I step in front of a crowd, with or without a musical instrument in hand. 


\section{TABLE OF CONTENTS}

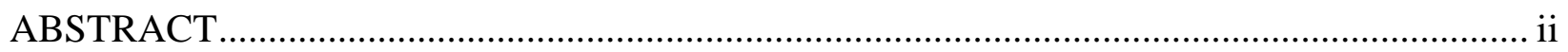

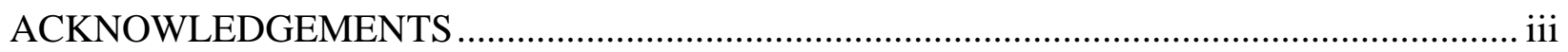

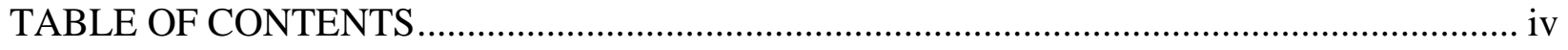

LIST OF FIGURES ….......................................................................................... vi

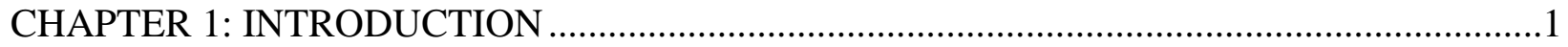

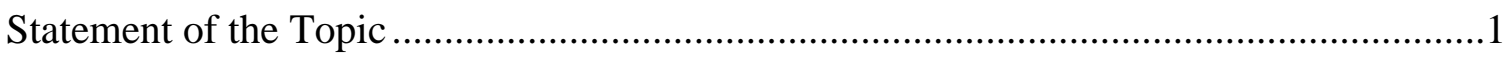

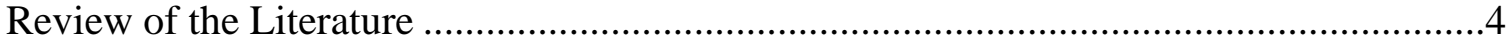

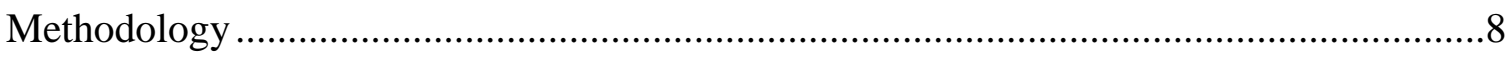

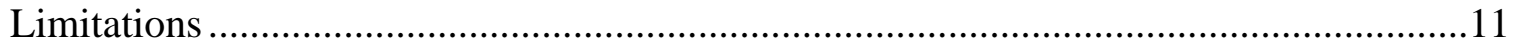

CHAPTER 2: EQUIPMENT AND SETUP ................................................................... 15

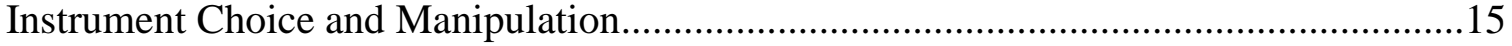

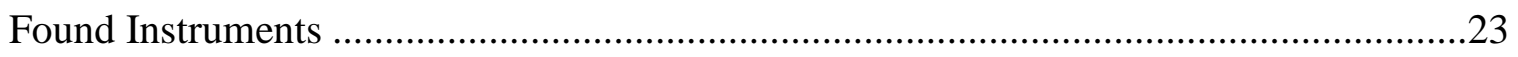

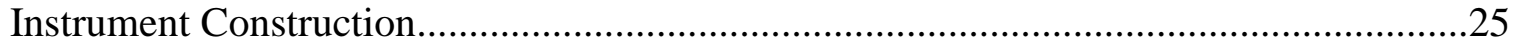

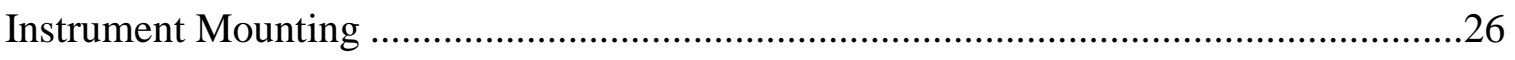

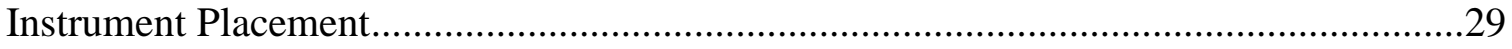

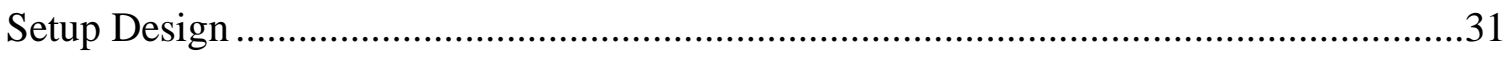

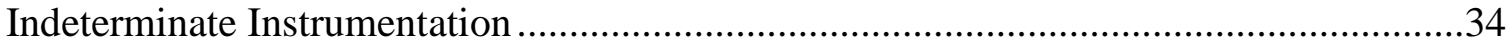

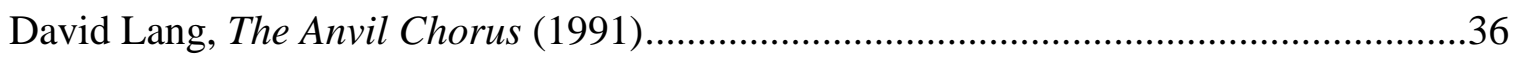

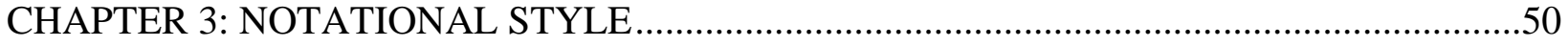

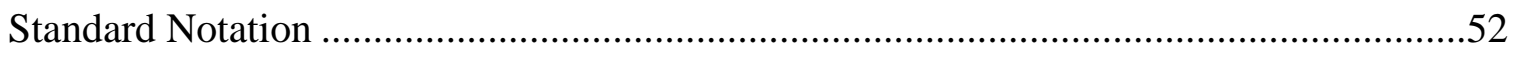

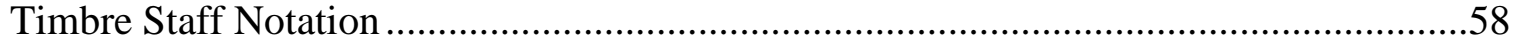

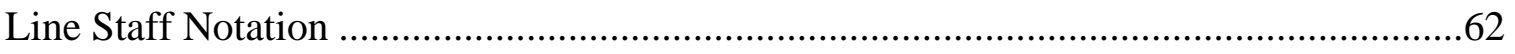

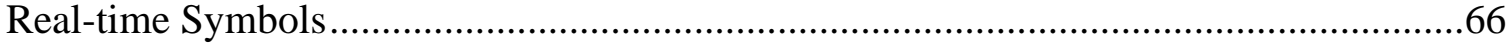

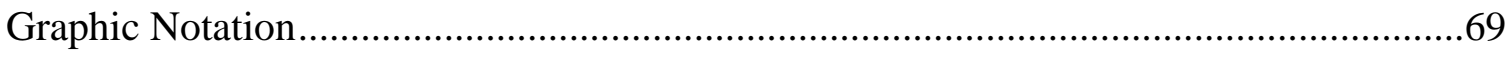

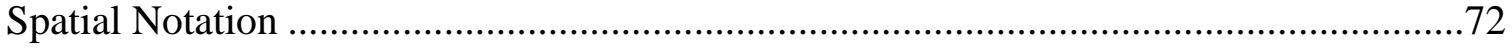

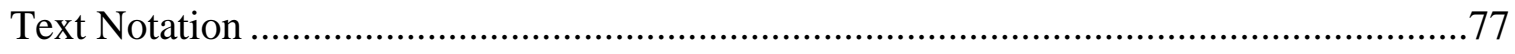

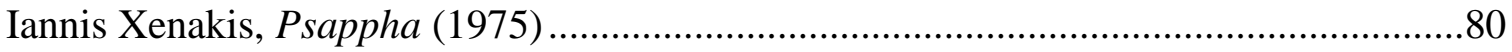




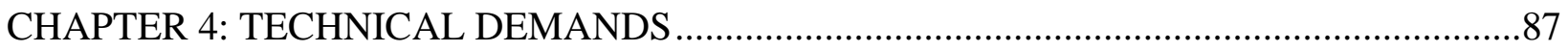

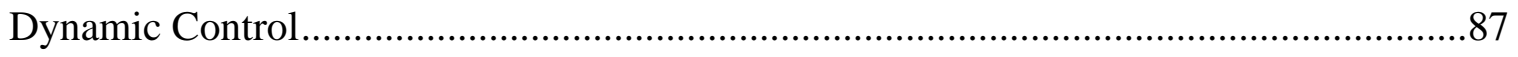

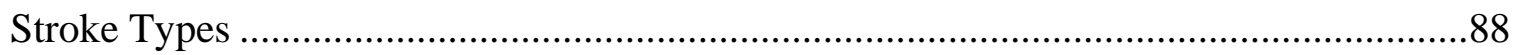

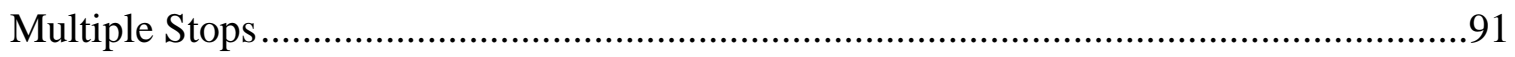

Coordination and Independence …………...........................................................93

Four-Mallet Grip ................................................................................................96

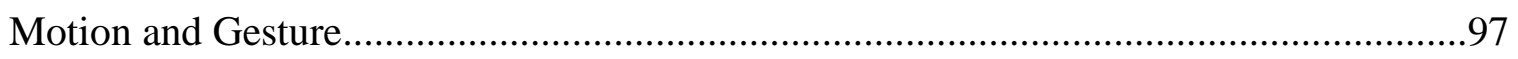

Maki Ishii, Thirteen Drums (1985) ..........................................................................100

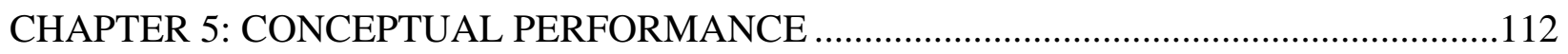

Gestures and Movement ……………………………….......................................115

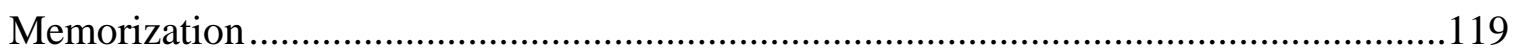

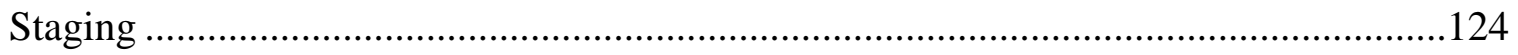

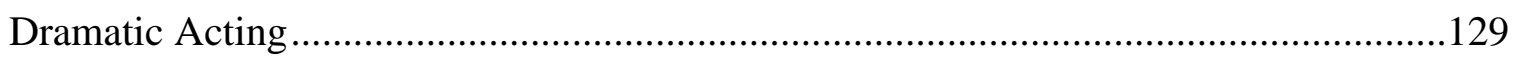

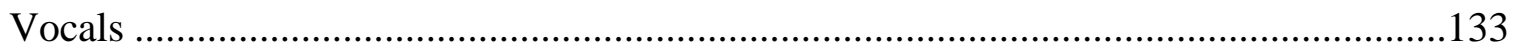

Stuart Saunders Smith, Tunnels $(1982$ - 5) .................................................................136

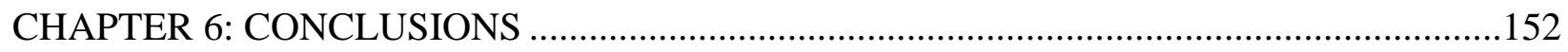

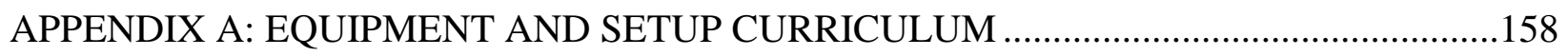

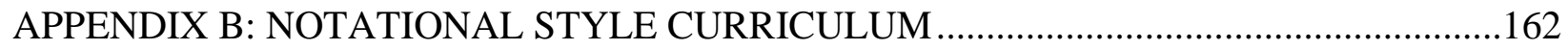

APPENDIX C: TECHNICAL DEMANDS CURRICULUM ....................................................167

APPENDIX D: CONCEPTUAL PERFORMANCE CURRICULUM ......................................170

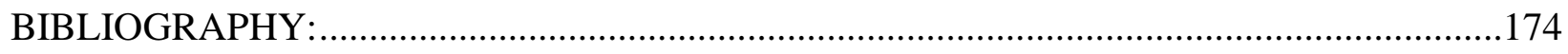




\section{LIST OF FIGURES}

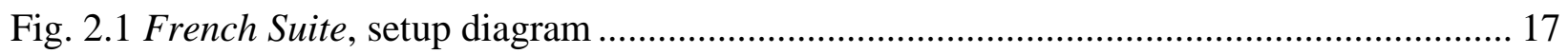

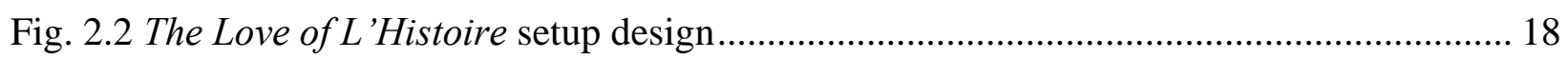

Fig. 2.3a The Love of L'Histoire, opening melodic theme in the wood group ............................. 19

Fig. 2.3b The Love of L'Histoire, rhythmic counterpoint of the cowbell ..................................... 19

Fig. 2.3c The Love of L'Histoire, harmonic double stop of bass drum and cowbell .................... 19

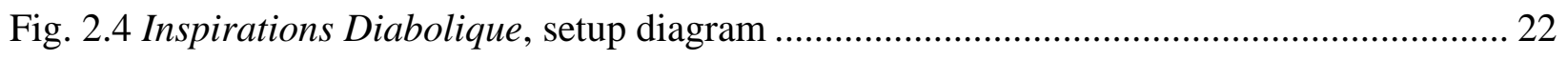

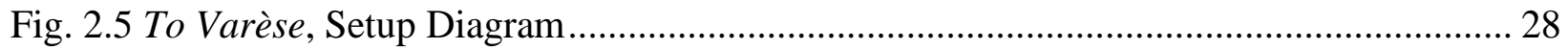

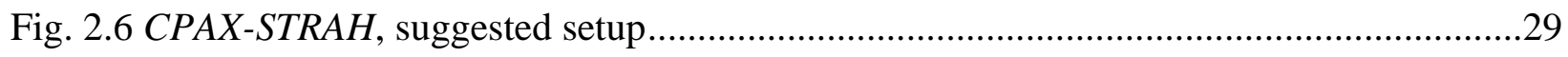

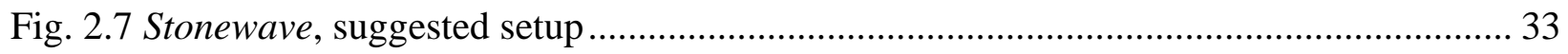

Fig. 2.8 Thirteen Drums, Berry "proximity-speed setup" ........................................................ 34

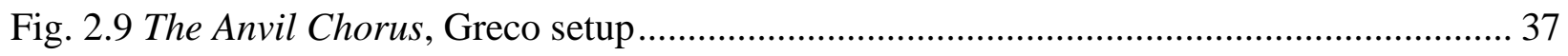

Fig. 2.10 The Anvil Chorus, instrument key ……………………………………..................... 38

Fig. 2.11 The Anvil Chorus, Greco setup for instruments struck with handheld mallets ............. 40

Fig. 2.12 The Anvil Chorus, Greco non-resonant steel plates....................................................... 41

Fig. 2.13 The Anvil Chorus, Greco stick tray .......................................................................... 42

Fig. 2.14 The Anvil Chorus, Greco setup of wood blocks ............................................................ 44

Fig. 2.15 The Anvil Chorus, Greco setup foot pedal cowbells .................................................... 46

Fig. 2.16 The Anvil Chorus, Greco foot pedals with marks........................................................ 48

Fig 3.1 The Love of L'Histoire, standard notation ............................................................... 52

Fig. 3.2 XII, standard notation without time signature and bar lines ......................................... 53

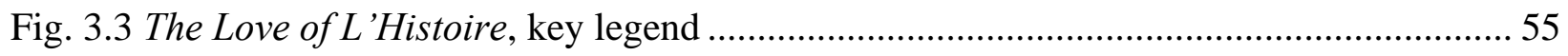

Fig. 3.4 French Suite I. Allemande, intermediate five-line stave .............................................. 56

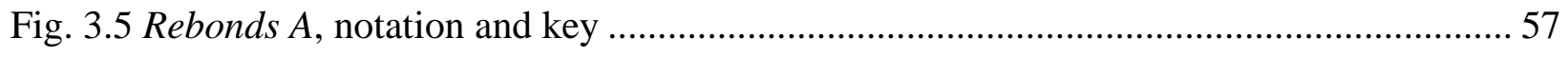

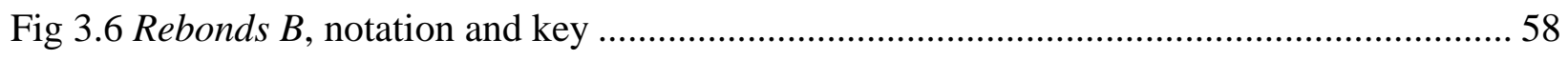

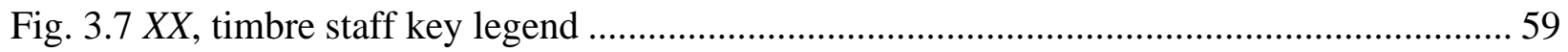

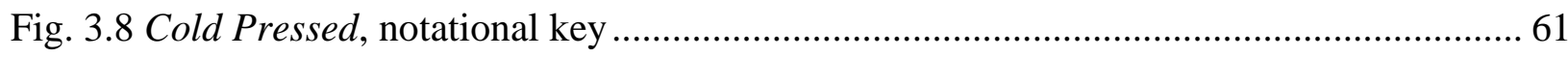

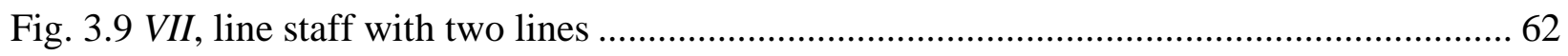

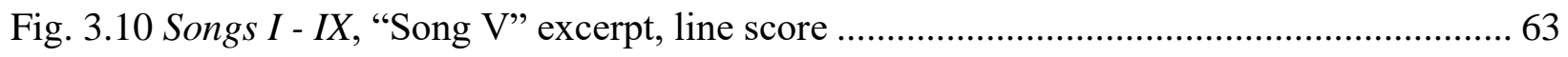

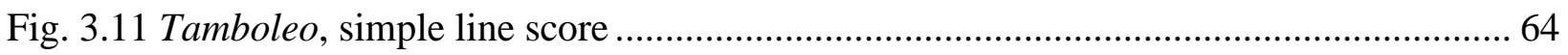




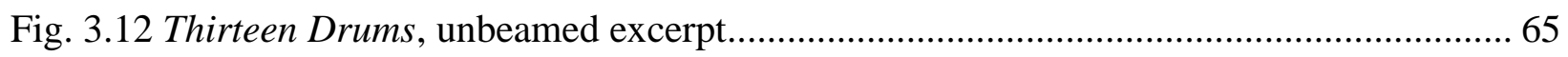

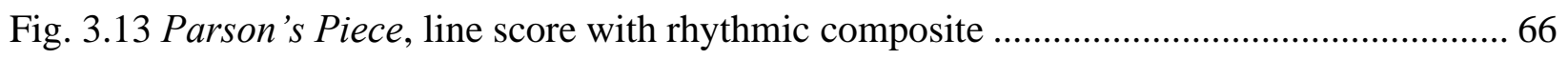

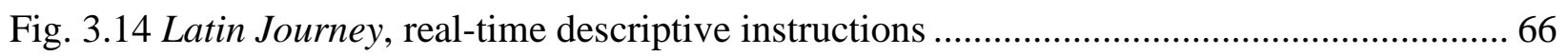

Fig. 3.15 Inspirations Diabolique, extended technique, implement, and note-head symbols ...... 67

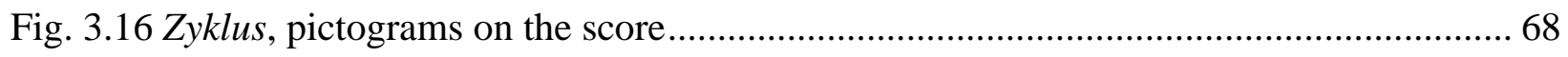

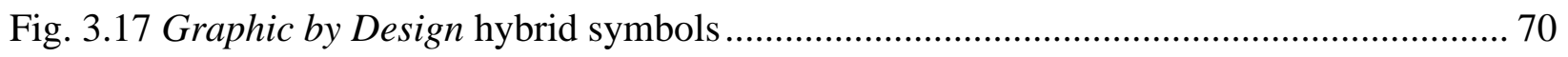

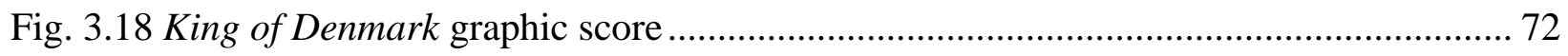

Fig. 3.19 XVIII, spatial treatment of rhythm ......................................................................... 73

Fig. 3.20 Spatial Choices, spatial treatment of pitch, dynamic, and rhythm ............................... 74

Fig. 3.21 27'10.554", spatial notational devices ...................................................................... 76

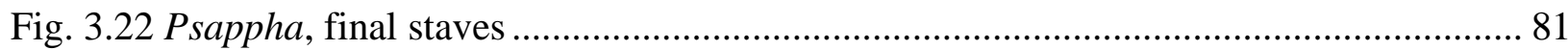

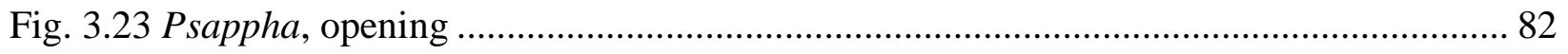

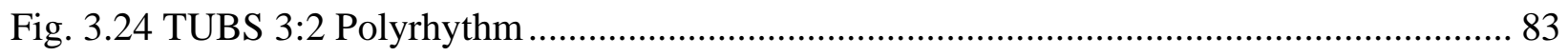

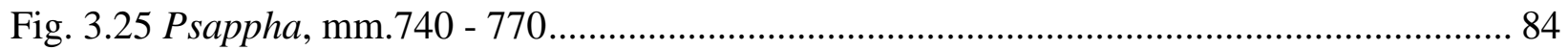

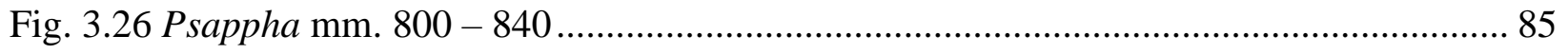

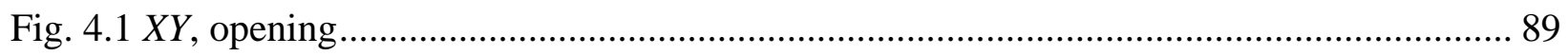

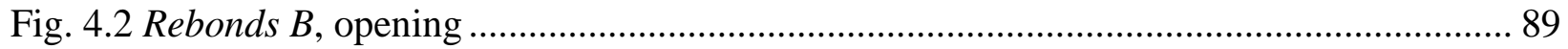

Fig. 4.3 Blade, double-stroke passage ……………............................................................... 90

Fig. 4.4 A Snare Growls, and He Flies, multiple-bounce fluency ............................................ 90

Fig. 4.5 She Who Sleeps with a Small Blanket, double-stop passage ......................................... 92

Fig. 4.6 Inspirations Diabolique, Movt. II. Dance, multiple-stop passage................................... 92

Fig. 4.7 The Anvil Chorus, finale (double-stop in the feet circled) ............................................ 93

Fig. 4.8 The Love of L'Histoire, tambourine ostinato .................................................................... 94

Fig. 4.9 Evil Ernie, simple independence …………............................................................... 94

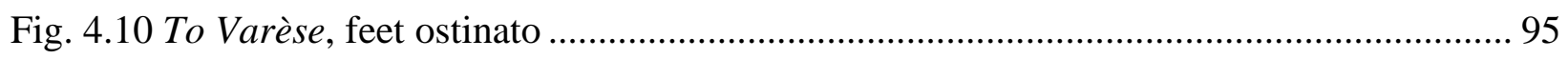

Fig. 4.11 Rebonds $B$, advanced orchestration independence ....................................................... 95

Fig. $4.12 X Y$, advanced polyrhythmic independence .................................................................... 96

Fig. 4.13 Psappha, excerpt utilizing 4-mallets ............................................................................... 97

Fig. 4.14 Stonewave, quick motion across large set-up ............................................................ 98

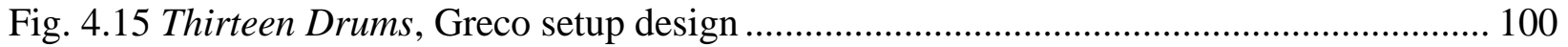

Fig. 4.16 Thirteen Drums, opening figure ............................................................................. 102 
Fig. 4.17 Thirteen Drums, first melodic phrase with three sticking models........................... 104

Fig. 4.18 Thirteen Drums, roll speed variation ............................................................... 108

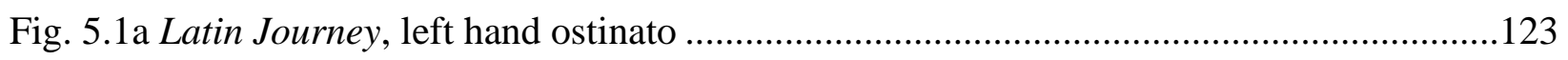

Fig. 5.1b Latin Journey, call and response ................................................................. 123

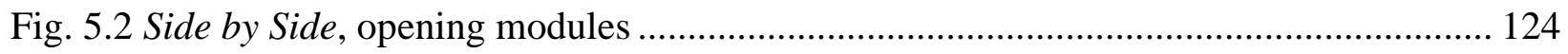

Fig. 5.3 ...And Points North, Movt. II, setup diagram ....................................................... 128

Fig. 5.4 The Discernment of Time, clock face association..................................................... 131

Fig. 5.5 Tunnels, lines 22 - 23, grace note symbol identification ........................................ 140

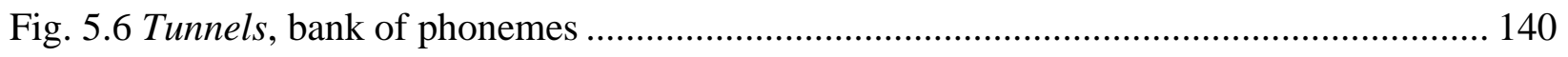

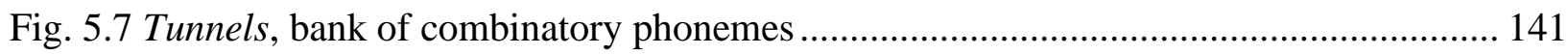

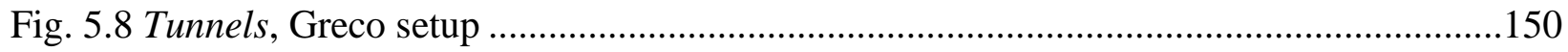

Fig. 6.1 Sample Master's Degree Program Curriculum ........................................................ 154

Fig. 6.2 Sample Doctoral Degree Program Curriculum .................................................. 155 


\section{CHAPTER 1: INTRODUCTION}

\section{Statement of the Topic}

Multiple percussion is currently an integral facet in the Western percussion art form, yet it still holds a precarious place in the collegiate percussion curriculum. Igor Stravinsky and Darius Milhaud were the foremost composers in demanding that percussionists play multiple instruments within a given piece or movement, a performance practice that would grow exponentially throughout the twentieth century. Even with the centennial of Stravinsky's Histoire du Soldat fast approaching, collegiate percussion programs continue to grapple with creating a standard curriculum that fits the needs of the modern percussionist playing multiple instruments. Pedagogical resources in multiple percussion are in relatively short supply as compared to those for mallet instruments, timpani and snare drum. The regularity of these core percussion instruments in various styles of music influenced early percussion programs across the country to enact curriculums, many of which became standardized and are still in use today. This should come as no surprise, since the first percussion program spearheaded at the University of Illinois in 1950 predates the solo multiple percussion medium. Despite its growing demand in real world situations within standard solo, wind band, and percussion ensemble repertoire, among others, multiple percussion may not be universally accepted as a core percussion instrument.

In its most pure form, the multiple percussion medium consists of a single percussionist playing multiple instruments in a single movement or piece. Those familiar with the practice recognize other challenges inherent to the medium, such as a unique legend that is needed to locate the specific instruments on the staff and the tendency for changing implements (i.e. sticks, mallets, hands) in order to produce the desired sound from each instrument. The frequent 
demand for its fluency in large ensembles, chamber groups, and percussion ensembles suggests that the development of multiple percussion skills should be fully incorporated into curriculum as a core instrument, not as an extracurricular medium but alongside snare drum, keyboards and timpani.

In addition to requisite skills essential for all percussionists, the solo medium has significant historic value: there are significant twentieth century composers that simply did not write for solo snare drum, keyboard percussion or timpani. For example, if a percussionist is to delve into the solo compositions by notable composers such as John Cage, Morton Feldman, and Iannis Xenakis among others, they must build their mastery in the realm of solo multiple percussion. Graduate programs may provide an opportunity to focus on obtaining fluency in the multifaceted aspects of multiple percussion through experiential learning of the repertoire.

This document aims to address a specific void in multiple percussion pedagogy through the creation of a multi-faceted model for performance practice. The model describes four dimensions that present specific demands required from the modern-day multiple percussionist: equipment setup, notational method, technical demands, and performance concept. Through the analysis of each dimension in relative isolation, the percussion instructor and student can better compartmentalize the demands that are placed on the performer in the medium.

Upon the description of each dimension, one piece is detailed as a case study, including my engagement with the piece in navigating and an analysis of its demands. Pedagogical considerations are also posited, such as development of the skill or concept, and the transferability to other pieces in the literature. This structure aims to help the percussion instructor build their multiple percussion curriculum so that students gain experience in working in each component. While students may seek to perform pieces that emphasize just one 
dimension, a percussion instructor can choose to provide a more comprehensive curriculum to prepare students for the breadth of the repertoire and demands of professional scenarios. Instructors may find that the resource helps build student outcomes for their applied lesson sequence through the isolation of each dimension, and students may find clarification of the multifaceted performance practice within this model.

The conclusion includes two curricula that I position as appropriate for a graduate student aspiring to become a multiple percussion specialist. The master's and doctoral programs are a significant stage in a percussionist's artistic training toward mastery of the craft, but there are currently few written resources solely dedicated to navigating the intermediate and advanced repertoire. The program curricula emphasize the interrelationship between the dimensions of multiple percussion, thereby using pieces that allow for developing multiple dimensions at a time, multitasking, and promoting a synthesis of information. In contrast, the appendices incorporate a series of suggested curricula, each dedicated to a specific dimension. These curricula emphasize the independence of each dimension, which suggest that by isolating certain problems, the instructor might create appropriate program goals and individualize student outcomes. They also show how specific pieces can be chosen to address deficiencies and inexperience, as well as those that play to a student's strengths. Each curriculum proposes a logical progression of pieces where each performance dimension can be developed, with a concise explanation of the demands of each piece

The format of the document is therefore in three parts: the (1) multi-faceted model, (2) case-studies, and the (3) proposed curricula. This format was chosen so that the model is clear for instructors, while also providing freedom so that the instructor can incorporate pieces from his or her own library to effectively build their own curriculum. The primary objective of the 
multifaceted model is to empower the instructor in building curricula that fits their needs and adapts to future changes in the multiple percussion catalogue. Although a multifaceted model never becomes outdated in its entirety, elements might be deemed ineffective now or in the future as a result of (a) additions to the solo multiple percussion repertoire, (b) an instructor's inventory, or (c) the availability of pieces due to publication changes.

\section{Review of the Literature}

Compared to the core instruments that are common in total collegiate percussion programs, there are few written resources that detail multiple percussion pedagogy and curriculum development. Experts have intermittently offered etude books that are aimed at the beginner to intermediate percussionist, with current offerings being made by Campbell/Hill and Bliss. ${ }^{1}$ In addition, a variety of DMA research documents highlight performance challenges for a limited number of pieces in the standard advanced repertoire. However, there is an absence of resources aimed at decreasing the distance between the intermediate and advanced repertoire. Udow's The Contemporary Percussionist should be familiar to aspiring multiple percussionists and percussion instructors; it occupies a unique position because the percussionist's music reading must be advanced, while those skills specific to multiple percussion remain at a relatively intermediate level. ${ }^{2}$

Julie Hill and James Campbell's Music for Multi-Percussion: A World View is a good example of an etude book that guides beginning and intermediate multiple percussionists. ${ }^{3}$ The collection is split into four main sections of etudes that emphasize specific skill sets: (1) a single

\footnotetext{
${ }^{1}$ James Campbell and Julie Hill, Music for Multi-Percussion: A World View (Van Nuys, CA: Alfred Publishing Co., 2008):

Andrew M. Bliss (ed.), Multitudes (Nashville: Innovative Percussion, 2009).

${ }^{2}$ Michael Udow and Chris Watt, The Contemporary Percussionist: 20 Multiple Percussion Recital Solos (Fort Lauderdale: Meredith Music Publications, 1986).

${ }^{3}$ Campbell and Hill, Music for Multi-Percussion.
} 
instrument or set of instruments, (2) changing implements on a single instrument, (3) multiple instruments and implements, and (4) three- and four-way coordination. This organizational method contributes to the pedagogical literature by introducing the technical demands of each etude. Despite the limitation of an intermediate grading level and relatively little prose instruction, etude books provide a precedent for the notated resources that imply a curriculum. These etude books typically address the beginning multiple percussionist and should be used within the first three years of undergraduate study, depending on such factors as the student's previous experience in multiple percussion and their interest, as well as program objectives and specialty of the instructor.

A few research projects closely parallel the present document, albeit utilizing a different methodology and perspective. Terry Applebaum details the notational methods and technical demands of five advanced multiple percussion solos: Circles by Lucia Berio, The King of Denmark by Morton Feldman, Liasons by Roman Haubenstock-Ramati, Quadrivium by Bruno Maderna, and Zyklus No. 9 by Karlheinz Stockhausen. ${ }^{4}$ The pedagogical focus in Applebaum's writing sets it apart from other graduate student research documents concerned with performance analyses of select pieces. The pedagogical focus emerged from his original etudes of graduated difficulty, geared toward the performance issues embedded in the five chosen pieces. The author recognizes that the individualized nature of the pieces would benefit from specified guidance and isolated skill development. The author does not formulate a curriculum, however, leaving space that I intend to fill with a significant feature of this document.

\footnotetext{
${ }^{4}$ Terry Applebaum, "A Comprehensive Performance Project in Percussion Literature with an Essay Comprised of Multi-Percussion Performance Problems as Found in Selected Contemporary Works, with Original Etudes Relevant to those Problems," (DMA document: University of Iowa, 1978).
} 
Benjamin Charles's doctoral document more directly addresses solo multiple percussion curriculum development, but within the scope of undergraduate percussion programs. ${ }^{5}$ It includes survey responses from instructors regarding general issues and trends in multiple percussion instruction at the university level. The author firstly describes his survey given to university percussion professors and an overview of the data collected from their responses. Charles proceeds to provide a repertoire guide for select solo pieces in the literature, categorizing works and building a system of grading difficulty primarily by the size of the setup. His rigorous research on equipment and setup dimension ultimately paved the way for the grander scope of the present document, including notational method, technical demands and conceptual components. Charles's undergraduate curriculum and proposed undergraduate recital sequences are also pioneering in how they highlight multiple percussion pieces that might fit students with various strengths and interests.

Other authors have also conducted research regarding advanced performance issues of multiple works in the performance practice. James Lambert chose five different works, limiting his research to three specific performance problems: notation, instrument placement (equipment and setup), and movement (part technical, part conceptual). ${ }^{6}$ Lambert thereby chose select aspects that occupy all four dimensions, albeit not with a comprehensive approach. Applebaum documented a select number of advanced pieces in the scope of notation and technical demands. By gathering and triangulating their research and blending in my own engagement with the

\footnotetext{
${ }^{5}$ Benjamin Charles, "Multi-percussion in the Undergraduate Percussion Curriculum," (DMA document: University of Miami, 2014).

${ }^{6}$ James Lambert. "Multiple Percussion Performance Problems as Illustrated in Five Different Works Composed by Stockhausen, Smith Brindle, Colgrass, Dahl and Kraft Between 1959 and 1969" (DMA document: University of Oklahoma, 1983), 5.
} 
repertoire, I am able to dedicate this document to exploring each of the four performance practice dimensions in detail and to construct a graduate level curriculum that addresses each.

A current trend in graduate documents details the performance issues for a piece or a select number of pieces in a repertoire. This is logical, as these students provide a written account of their preparation of piece(s) that fulfill the performance recital requirements for the degree. This approach has resulted in many individual interpretations of standard pieces in the repertoire, which provide insightful performance analyses of the works. The primary focus of research in this realm appears to be the analysis of the pieces themselves. My project differs in that the primary objective is the description of the dimensions of performance practice and graduate level curriculum, which is only illuminated through the intensive analysis of how a significant piece exemplifies that particular component.

Two available books that introduce concepts of multiple percussion medium are Nick Petrella's The Multiple-Percussion Book: Concepts for a Musical Performance and Steven Schick's The Percussionists Art: Same Bed, Different Dreams. ${ }^{7}$ Petrella maintains a focus on performance practice, introducing beginning concepts such as instrument height and position of the music stand in the first part of the book. This leaves only brief remarks, if any, for intermediate and advanced concepts before the book progresses to newly composed etudes by Jon Allemeier. Petrella does not correlate how these concepts apply to specific pieces in the standard repertoire. On the other hand, Schick maintains his conceptual perspective through the description of monumental pieces in the repertoire. He allows only secondary focus to performance analysis of select advanced works, such as instrument setup, notation, and technical

\footnotetext{
${ }^{7}$ Nick Petrella and Jon Allemeier, The Multiple-Percussion Book: Concepts for a Musical Performance (New York: Carl Fischer, 2000):

Steven Schick, The Percussionist's Art: Same Bed, Different Dreams (Rochester, NY: University of Rochester Press, 2006).
} 
skills. Despite the primarily autobiographical nature of the work, The Percussionist's Art has enormous value for performers and instructors alike, mainly from the author's extensive experience as a leading performer, his dedication to new commissions, and his ongoing dialogue with significant composers. Neither Petrella nor Schick discuss curriculum development, a primary aim of this document.

\section{Methodology}

The methodology for the project began with detailing specific performance practice dimensions I have found to be requisite skills and concepts for percussionists. Upon analysis, performance, and coaching of students through intermediate and advanced repertoire, I discovered four major performance dimensions. Much testing and reflection has been given to how a percussionist might develop those skills, which will be further analyzed through the lens of a specific piece in the repertoire. The pieces were chosen because I have performed each of them in the solo setting in my DMA program, allowing me deeper knowledge and affiliation that will guide the analysis where necessary. I selected pieces that span the intermediate to advanced difficulty range and analyzed them in the scope of each performance dimension, ultimately categorizing them based on their treatment of the four dimensions. Upon the creation of a repertory of each dimension, I analyzed each piece for difficulty to posit curricula geared toward each component. I gathered appropriate pieces from my own collection, the West Liberty University percussion department library, other libraries via interlibrary loan, and rentals to acquire the sheet music for this study.

The base of the research assumes that the percussion student has the requisite skills to perform Charles DeLancey's The Love of L'Histoire (1973). The work was one of few mentioned by multiple college percussion instructors as significant pieces in the multiple percussion repertoire in Charles's survey, one undergraduate percussion majors should 
reasonably perform with precision. ${ }^{8}$ Its historic value lies in its homage to the chamber piece with which the solo work is based, Stravinsky's Histoire du Soldat. It is also an interesting adaptation of the chamber work for the solo percussion medium, orchestrating important themes from the wind and string parts. From a performance standpoint, the solo work is a significant intermediate work in each of the four dimensions. It calls for varied instrumentation of skin, metal, and wood surfaces, covering a wide area that spans the horizontal and vertical planes. The notational style is at an intermediate level as well: the different staves organized by instrument material requires an element of awareness and will pose a learning curve, but the standard rhythmic notation presents no challenge for the percussionist. Technical demands are also appropriate for undergraduate recitals, including that the piece is certainly longer than any etude, the detailed playing spots, double-strokes, and rapid shifting between instruments required, most notably in the Triumphant March of the Devil finale. In terms of performance concept, the intermediate percussionist is challenged to create melodies between the surfaces of different material and to maintain a sensitive balance between the different instruments that occur simultaneously. The student should be encouraged to conduct independent research on the original chamber piece to effectively portray the style changes between the different movements. While such a display of style change is relatively advanced, the fermatas and pauses between the such changes allow the intermediate percussionist to regroup themselves before significant changes. In each of the four dimensions, Charles DeLancey's The Love of L'Histoire is the epitome of an intermediate piece worthy of undergraduate recitals. I will be assuming that the percussionist has the requisite skill to perform the piece, and therefore will begin my graduate level curriculum with it.

\footnotetext{
${ }^{8}$ Charles, "Multi-Percussion in the Undergraduate Percussion Curriculum," 28.
} 
I found that the (1) equipment setup, (2) notational style, (3) technical skills, and (4) performance concept each present distinct challenges for an effective performance. The equipment setup dimension includes requirements such as the instruments needed, the amount of setup detail provided in the score, problems with indeterminate instrumentation, construction of instruments and setup apparatuses, and the amount of space needed. David Lang's The Anvil Chorus (1991) provides an excellent case study for the equipment dimension. A performer's perspective of various notational systems is provided, as it is a significant part of learning and performing a work. The application of standard, line-score, time-unit box, graphic, and temporal notational elements are described, ultimately positing the effectiveness of the notation and its potential learning curve. Iannis Xenakis's Psappha (1975) is described in the lens of notational method. Discussion of the technical demands includes the required stroke types, changes of technique based on different surface material, movement between instruments, coordination and independence, implement changes, endurance, and specified playing spots. Maki Ishii's Thirteen Drums (1985) is analyzed through the technical lens. The performance concept dimension pulls from aspects of musicality (phrasing, articulation, dynamic changes and control, tempo changes and rubato), expression (memorization/internalization, gesture), and extramusical concepts (voice, staging, logistics, lighting, costumes and props). Finally, Stuart Saunders Smith's Tunnels is used as the case study for the conceptual dimension, chosen because the percussionist serves as part composer, actor, and narrator.

Upon constructing the model and reflecting on my experiential learning via the four advanced pieces, I returned to the music scores with the specific intention of creating the curricula. Readers are reminded that the curricula for the degree programs can be found in the conclusion, and the curricula specific to each dimension can be found in the appendices. I have 
evaluated over fifty pieces in terms of their difficulty in each dimension, ultimately using over thirty in my curricula. In general, I found exemplary works to introduce a particular facet, along with pieces that challenge and those that even require mastery of that facet. From this research I created a logical course of their study to encourage a clear compartmentalization and a succinct development of the components of the multiple percussion performance practice.

\section{Limitations}

There were many limitations and other difficulties in this project, which has likely contributed to the persistent void of this type of document within the multiple percussion pedagogy literature. To those well-practiced in the multiple percussion medium, the individualized nature of each piece in the repertoire is apparent. The core percussion instruments, like others in the Western art music idiom, enjoy a high degree of standardization; there are notational, technical, and musical similarities in the repertoire that point to a clear continuum. Numerous and overt points of succession might suggest a more intuitive curricula: keyboard study generally begins with two-mallet study on a four-octave marimba before "graduating" to four-mallet technique on a 5-octave marimba, and timpani study typically begins with just two pitches on two drums before delving into changing pitches on three or more drums. Despite differences in style within the literature, percussionists generally feel assured that the development of skills and knowledge learned in the preparation and realization of one piece can transfer to the next. On the other hand, there is a less discernable link between pieces for multiple percussion, a consequence of the individuality of instrumental setup, notation method, techniques, and conceptual properties within the medium's vast musical style. While the unique qualities of each piece and individual interpretations by each performer may appeal to some musicians, it has likely influenced performer-scholars avoiding developing universal curricula 
concepts. A primary objective of this research document is to clarify possible links between pieces for multiple percussion solo, so that the instructor can choose the optimum literature. In addition to the challenges of this study, there were several limitations. Even in its relatively short lifespan, the sheer scope of solo multiple percussion literature can be quite overwhelming. I searched for works that utilized a unique set of multiple instruments, which includes those of homogenous and heterogenous materials (wood, metal, skin, etc.). Although keyboard instruments and timpani incorporate multiple surfaces (bars, drums), their conventions of pitch and harmony structures, instrument setup and notation runs counter to multiple percussion performance practice.

Beyond the sheer number of instruments, the multiple that differentiates multiple percussion from other aspects of percussion may also refer to the number of implements needed for the piece: various sticks, mallets, beaters and even a player's hands. Multiple may also refer to the multiple playing spots specified for the musician. Implement and playing spot changes construct a larger vocabulary of sounds than conventional performance, regardless of the instrument setup. A less intuitive dimension to multiple percussion may be its inherent degree of individualization in its notation. Unique notations result not only from the individuality of instrumentation between pieces in the medium, but also from modern composers' experimentations, which grapple with musical conventions of pitch as the sole arbiter of musical form and structure. According to this criterion, pieces scored for a single instrument can still be considered multiple percussion, such as James Tenney's Having Never Written a Note for Percussion (1971) for solo tam-tam and the entire The Noble Snare (ed. Smith) collection, even though the instrumentation is primarily for a solo snare drum. Similarly, I have chosen to 
exclude works for solo drum set, due primarily to the relative standardization of instrument setup and notation style and the thoroughness of Kevin Nichols and Daniel Adams. ${ }^{9}$

In a similar fashion, percussion typically holds a unique position to the concept of solo performance practice. For other instrumentalists, as well as vocalists, a solo work may very well incorporate an accompanist. It is very common for a solo recital to include an accompanist for every piece, but the same is not currently true for the percussionist. While I encourage the incorporation of collaborative musical practices into percussion performance practice and academia in general, chamber works and accompanied solos lie outside of the scope of the study. This exclusion was applied to works with pre-recorded or live electronic accompaniment, considering that the additional compositional, technological and sensitivity demands are best addressed elsewhere. ${ }^{10}$

Another problematic factor in the multiple percussion literature is the appropriateness of the work for a collegiate recital. Since percussion programs typically require a form of breadth in the material presented at recitals, pieces of incredible length are impractical even for graduate student recitals. Despite the significance and genius of the work, John Luther Adams's The Mathematics of Resonant Bodies would only appear on a student recital in an extremely exceptional case, given its performance time of over an hour. Pieces that specify a certain landscape for performance are unrealistic for student recitals that are restricted to a recital hall on campus, an example being the amplified snow required for Matthew Burtner's Syntax for Snow.

\footnotetext{
${ }^{9}$ Kevin Nichols, "Important Works for Solo Drum Set as a Multiple Percussion Instrument," (DMA document: University of Iowa, 2012):

Daniel Adams, "The Drum Set as a Solo Multiple Percussion Performance Medium," NACWPI Journal 52, no. 3: 4 -13 .

${ }^{10}$ Eric Derr, "Contemporary Percussion in the Age of Information Overload: Three Pieces for Percussion and Tape," (DMA document: University of California, San Diego, 2014).
} 
Consequently, works for specific landscapes and outstanding length were not considered for this study.

Lastly, the sheer number of case studies is a limitation for a project of this scope. There are many works that may be used in the curriculum at the graduate level depending on the individual needs of the student. Still, the discussion of the case studies will highlight appropriate concepts for instructors to consider, no matter what piece of the repertoire they may choose in the future. In addition, my analysis will be primarily in the realms of performance practice and pedagogy, and will not consider various historical, theoretical or socio-cultural analysis that may prove significant to the performer-scholar. 


\section{CHAPTER 2: EQUIPMENT AND SETUP}

The ability to navigate through equipment and setup challenges is an obligatory skill for the successful multiple percussionist. This dimension is chosen as foremost not only because equipment and setup decisions are likely a prerequisite to begin learning a piece, but also due to the significant implications it has on the other aspects of the solo multiple percussion medium; instrument choice and design directly affects the performer's comprehension and interpretation of the notation, the technical demands necessitated for performance, and the parameters of portraying a conceptual performance. One might argue that technical and expressive prowess

will mean little in one's realization without the proper consideration of the equipment and setup. Facets of the equipment and setup dimension include instrument choice, manipulation, construction and substitution, as well as elements of instrument design such as placement and mounting. These skills can best be developed in students when considered mindfully and included in the instructor's pedagogy and curriculum.

\section{Instrument Choice and Manipulation}

Choosing the best instruments that produce the desired sound is a crucial skill for the multiple percussionist. While instrumentation is inherent in other core instruments, multiple percussionists must consider sound properties such as frequency (pitch), amplitude envelope (articulation), and amplitude (dynamic) when constructing their instrumentations. When the available equipment does not precisely make the desired sounds, techniques of manipulating the instrument should be considered.

While not providing overt timbre sophistication, pieces with relatively homogenous instrumentation provide a clear opportunity for the student to explore tuning possibilities. William Kraft's French Suite is an intermediate piece for tenor drum, field drum, two snare 
drums, bongos and two cymbals (Fig. 2.1). ${ }^{11}$ Like The Love of L'Histoire, the setup is also "derived from the percussion part for Stravinsky's Histoire du soldat," but without the accessories and the large bass drum. French Suite will likely not be a difficult undertaking for the student who has already performed The Love of L'Histoire, their notational and technical demands are comparable as well. However, preparing the Kraft piece provides an opportunity to focus on the specific aspects of drum choice and tuning. Kraft's setup design and notational style clearly point to sequential pitch structure, which will allow the student to concentrate on other sound property details as well, including the resonance of each drum and the relationships of pitch (interval) and resonance between all drums. ${ }^{12}$ For example, bongos may not only have the highest pitch of the group, but should also have the shortest duration of resonance due to being single-headed drums with relatively thick drumheads. Therefore, the performer may wish to construct a continuum of resonance between the instruments, in which case each drum will have progressively longer resonance as they move lower in pitch. Synchronizing pitch and resonance strengthens their "internal coherence" and the perception that a listener will consider the homogenous instrumentation as a single unit. ${ }^{13}$ The tuning technique also widens the spectrum available to the palette of sounds with extreme clarity: without deviation, the lowest drum has the longest sustain and the highest drum has the shortest. In this case, tuning the tenor drum to have its longest possible resonance is achieved by using a double-headed drum, both equipped with

\footnotetext{
${ }^{11}$ William Kraft, French Suite for Percussion Solo (Bryn Mawr, PA: Theodore Presser Co., 1962).

This first publication does not provide a clear setup diagram that can be applied to all movements. This setup diagram is from Davis, "Theories of Rhythm and Meter and Their Pedagogical Implications for Non-Pitched Percussion Music with an Analysis of William Kraft's French Suite," (DMA document: University of South Carolina, 2011), 36.

${ }^{12}$ Kraft does not articulate at the onset of the piece, but the key legend for Movement III. Sarabande provides a note that the drums are graduated low to high.

${ }^{13}$ Defining aspects of "internal coherence" given in Schick, 17.
} 
single-ply heads that are identically tuned, causing their sympathetic vibrations to encourage each other.

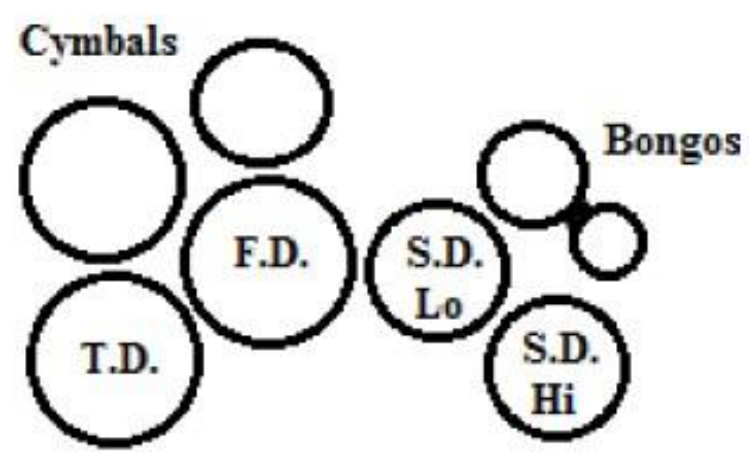

Fig. 2.1 French Suite, setup diagram

The complexity of the sound properties in percussion, or any acoustic sound for that matter, goes well beyond any type of written notation system. Interestingly, Adams found that written notation has rendered a general timbral deficiency among Western percussionists as compared to their counterparts in Indian, Japanese, and Islamic drumming. He suggests that percussionists adopt a syllabification or vocalization system to internalize timbral contrasts, an advanced audiation skill that would suit the percussionists' early stage of choosing implements and instruments. ${ }^{14}$

When surveying possible instruments in the preparation for The Love of L'Histoire, I always suggest my students first audiate the type of sound that he or she desires for the instrument. While choosing the three wood blocks might be intuitive, close attention should be taken in orchestrating the temple block. The student should ask why DeLancey chose to orchestrate three wood blocks and one temple block, rather than four wood blocks. What is the difference of sound between those two different instruments? The simple answer is that a temple block provides a warmer, darker sound with its fundamental frequency more emphasized than

\footnotetext{
${ }^{14}$ Daniel Adams, "Striking Implement and Surface Area Specification,” 21.
} 
manufactured wood blocks. Therefore, if a temple block is not available to the student, then it should be substituted with a "woody" sound that not only is lower in pitch, but also produces a warmer quality.

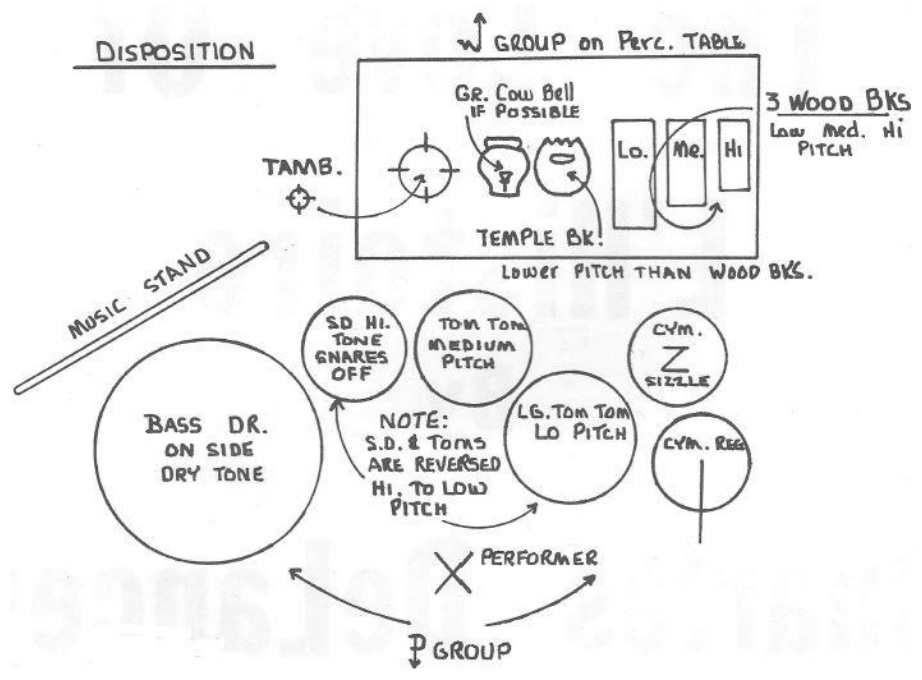

Fig. 2.2 The Love of L'Histoire setup design

The composer explicitly directs the performer to orchestrate with a "German" cowbell, reinforced through the pictogram that resembles an almglocken (Fig. 2.2). How does the sound of an almglocken differ from an African diaspora cowbell? For the opening theme (Fig. 2.3a), the temple block and cowbell should both contribute to the sequential pitch structure with the woodblocks to create a melodic line that imitates the brass opening of Stravinsky's Histoire du soldat. The instruments will be perceived as a melodic unit if other variables, such as timbre and resonance, are consistent throughout the group. In other words, if the cowbell is far more resonant and open than the wood and temple blocks, then it will appear to have a different role, one that does not equally contribute to the melodic contour of the other instruments. In this way, muffling the cowbell would be an appropriate manipulation that can be done by (1) affixing moleskin to the instrument, (2) laying the instrument on a surface, (3) placing a towel over a portion of the instrument, or (4) a combination thereof. 


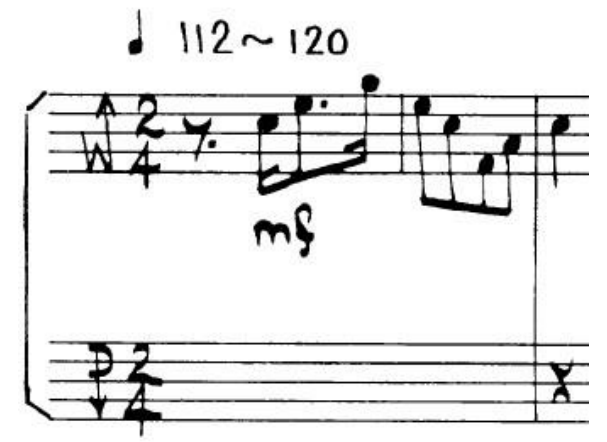

Fig. 2.3a The Love of L'Histoire, opening melodic theme in the wood group

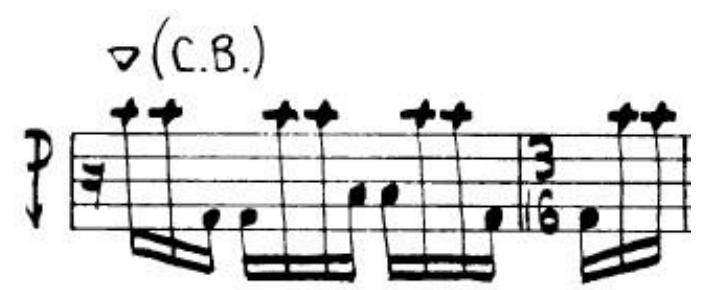

Fig. 2.3b The Love of L'Histoire, rhythmic counterpoint of the cowbell

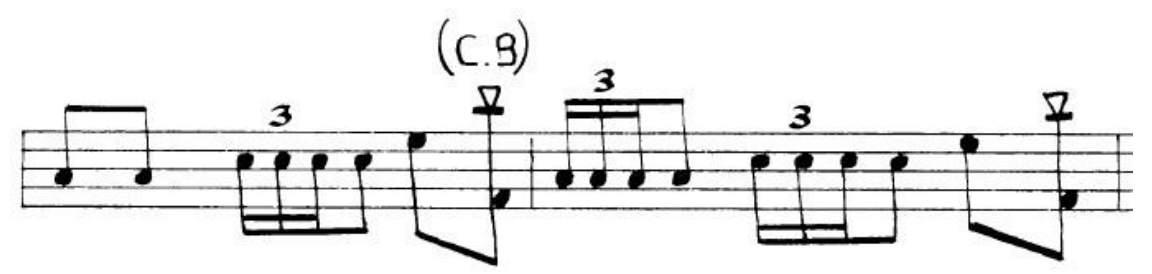

Fig. 2.3c The Love of L'Histoire, harmonic double stop of bass drum and cowbell

The student should also survey the other musical roles that the cowbell has in the piece, which would illuminate that it also (1) serves as rhythmic counterpoint with the drum group (Fig. 2.3b) and (2) performs harmonic double stops with the bass drum, adding percussive noise to the accelerando of the finale (Fig. 2.3c). DeLancey chooses three different placements of the cowbell between the two notation staves and two different note-heads in various sections, perhaps alluding to the roles the instrument holds. In this way, it is possible that the percussionist might best serve these roles by (a) choreographing a change in its manipulation (muffling) within the piece and/or (b) doubling the instrument by placing another cowbell elsewhere in the setup. Applying a piece of moleskin, gauze, or similar apparatuses to the instrument can diminish 
unwanted overtones and create a remarkably different sound. Interestingly, neither the temple block nor cowbell was scored by Stravinsky in Histoire du Soldat, bringing the student's creativity and problem-solving to the forefront. In summary, the student should carefully listen to each instrument in isolation and within their actual instrument combinations, which many manifest both melodically and harmonically. These multiple types of listening should be done in tandem, allowing each to influence one another in order for appropriate instrument choice and manipulation changes to occur. In general, altering the sound properties of available instruments will allow the percussionist to create different timbres with the instruments already in their collection.

For instrument choice and manipulation, the score will inherently provide a varying amount of implicit and explicit directions to the performer. How notation style factors into this interpretative process is discussed further in Chapter 3. For our purposes here, the level of explicit directions in The Love of L'Histoire presents an intermediate task for the performer. The percussionist is provided with details regarding instrument choice and a detailed setup design ("disposition") that guides logistic and interpretative decisions such as resting the bass drum "on side" and having a "dry tone," the snare and toms "are reversed hi. to low pitch," and that the temple block is "lower pitch than wood bks" (Fig. 2.2). Such explicit guidelines are not uniformly presented in the advanced solo repertoire, nor are they provided with such detail within other musical contexts, for that matter. Thereby, such explicit directions should be consciously negotiated, making way for progressively advanced implicit directions throughout the percussion curriculum.

Rickey Tagawa presents a logical next step in navigating explicit and implicit instrumentation directions in Inspirations Diabolique (1965). Tagawa directs the general position 
of the instruments in his setup diagram (Fig. 2.4), but the performer will need to place their instruments with more detail to minimize dead space and maximize their efficiency of movement. Unlike DeLancey, Tagawa does not offer suggestions in terms of means or hardware to suspend the instruments. Intuition and creativity will be needed for each individual design, as the bass drum and field drum are notably illustrated to have strikingly smaller diameters in the diagram than the actual instruments. Tagawa explicitly directs the pitch framework within same instrument groups (i.e., low and high snare drum, low and high cymbal), but he does not between heterogenous groups of instruments.

Inspriations Diabolique demands the percussionist consider timbre and melody within their instrument choice and manipulation. Since the jingles blur the pitch of the instrument and create a different timbre, the student will have to interpret how the tambourine fits into the pitch structure of the rest of drums. If a performer perceives the tambourine as a contributing member of the drum family, a single-row tambourine should be used. The perspective that the tambourine provides a distinct color would warrant using a double-rowed tambourine that emphasizes that aspect of the sound. The choice of tambourine, as well as its mounting strategy, also requires special attention because it is to be removed and placed on the low bongo for the second movement. Timbral variety within the drums also surfaces when considering snare drums; by way of comprehensive score study, the percussionist finds that both snare drums have occasions where they are played with snares off, bringing significance to their tuning relationship within the drum group. The relationship between the "A" and "B" sets of cymbals also demands consideration, through score study we find that they are not played within the same movement. The A set of cymbals are used for the introduction movement, where we find them scored with articulate quarter notes. The B set of cymbals are used for the later tarantella movement, in 
which case the cymbals are fluidly rolled. In order to bring out the musical roles that the sets of cymbals have, it would be appropriate to equip the A cymbals with those that activate fast and decay quick, versus the B cymbals that sound rounder and sustain longer.

INSTRUMENTATION DIAGRAM

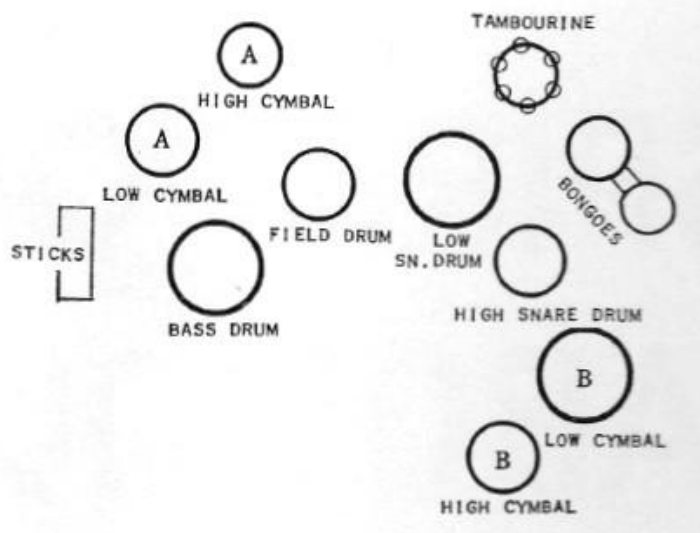

Fig. 2.4 Inspirations Diabolique, setup diagram

Triangulation of the diagram illuminates a sequential treatment of the drum group from left (bass drum) to right (small macho bongo). Intuition will lead the student to treat the pitch component from lowest to highest, but other sound properties should be applied as well. In terms of resonance, one could tune the bass drum to have the longest sustain, moving sequentially to the shortest sustain in the high bongo. Tonality can be applied to the sequence by tuning the bass drum (i.e., the resonant head), since it has the most indefinite pitch in the setup, moving to the highest bongo that has the clearest tone. Listener's perception of tonality could be influenced by sequencing: (a) the size of the intervals between instruments, such as the largest interval being orchestrated between the bass drum/field drum and the smallest between the low bongo/high bongo, and/or (b) the relative consonance and dissonance between instruments, such as orchestrating a P5 between the lowest two drums and moving to a $\mathrm{m} 2$ between the highest two. These details describe opportunities for sympathetic navigation of the composer's intentions via 
the implied directions, which could ultimately lead percussionists to a variety of different conclusions.

Inspirations Diabolique works well as an intermediate-advanced piece within the equipment and setup dimension because students can explore these permutations within the homogeneous instruments. A minimal setup of other timbres is present in the tambourine and cymbals, which allows the student to engage in a heterogenous setup without being overwhelmed. The ethereal style and open musical texture in much of the piece allows the student an opportunity to listen to their sounds with intent. Percussionists can explore sound properties and navigate implicit directions while still being guided with explicit directions within a relatively small setup of instruments that are standard to percussion inventory. This is what makes Inspirations Diabolique a logical next step in the equipment and setup dimension.

\section{Found Instruments}

Fluency regarding the use of found instruments, or found objects, is an obligatory skill of the modern percussionist. There is validity in building an appreciation of the historical significance that found objects had in the early years of percussive art music, but also its continued place in current compositions for solo and ensemble works, as well as improvisational music, general music education and other contexts that are applicable to the modern world.

Welwood defines found instruments:

"and ready-made object that is capable of producing a sound and that has been discovered (or found) to be musically valuable... a found instrument differs from a conventional one in that it is usually simpler in construction and in its ready-made form is not usually associated with the music of a particular culture." 15

Welwood continues to articulate various benefits of implementing found instruments into the music classroom, which include students' exploration of sound properties, group improvisation

\footnotetext{
${ }^{15}$ Arthur Welwood, "Improvisation with Found Sounds," Music Educators Journal 66, no. 5 (January 1980): 72.
} 
possibilities and the development of intrapersonal skills that are learned when using own's own body as an instrument. ${ }^{16}$

Beginning in 1984, Percussive Arts Society sponsored a regular column in Percussive Notes called "Instrument Innovations," edited by Jon Scoville. The column was aimed at exploring innovative instrument makers and unique examples that Scoville posits may have represented a counter-reaction to the rise of electronic music. According to Scoville:

"a vital resurgence in the invention of new instruments, in the construction of 'sound sculptures' - objects which combine both visual and sonic properties in a unique fashion, and in the utilization of 'found sound' - items found anywhere from kitchens to junkyards which make new sounds from familiar objects."17

In addition to the regular column, Scoville and Banek's Sound Designs, as well as resources by Hopkin and Grayson may be useful for those interested in the design and construction of instruments. ${ }^{18}$ Of course, the maturity of self-made percussion instruments is seen in the refined musical constructions of Harry Partch in the mid-twentieth century.

Given its significance, found instruments should be integrated into the collegiate percussion curriculum. In this context of early percussion ensemble literature, consider namely John Cage and Lou Harrison, this appears widespread across collegiate programs. Yet exploring found instruments as a soloist may present further problems, such as the vulnerability of performing alone and negotiating the duality of serving as an expert authority presenting a formal recital while performing on a collection of instruments that could be found at home, in nature, or the local hardware store.

\footnotetext{
${ }^{16}$ Ibid., $72-77$.

17 Jon Scoville, "Found Sound," Percussive Notes 23, no. 1 (October 1984): 43.

${ }^{18}$ Reinhold Banek and Jon Scoville, Sound Designs (Berkeley, CA: Ten Speed Press, 1995):

Bart Hopkin, Musical Instrument Design: Practical Information for Instrument Making (Tucson, AZ: See Sharp Press, 1996):

John Grayson, Environments of Musical Sculpture You Can Build (Vancouver: Aesthetic Research Centre of Canada, 1976).
} 
To the Earth (1985) by Frederic Rzewski is an exemplar found instrument piece for the aspiring advanced multiple percussionist. Like all his works, Rzewski has made the score available free online. ${ }^{19}$ The piece calls for four flowerpots and requires the percussionist to recite text. According to Schick:

"Rzewski told me in conversation that the pitches he indicated for the flowerpots - one of the few truly musical elements in the piece-are not sacred. Change them if you like, he said." 20

Provided with the freedom of pitch, the percussionist is free to focus on obtaining flowerpots that produce the desired sound and dramatic effect. Influenced by his communication with Rzewski, who described a desire for the performance to be "common and accessible rather than exalted and artistic," Schick offers suggestions of flowerpot choice, implement and treatment of the text. The true difficulty of the piece is synchronizing the flowerpot and the recitation of the text appropriately, sounding and feeling like independent voices when necessary.

\section{Instrument Construction}

A percussionist will undoubtedly be called on to construct an instrument some time in their career. Notable examples of instruments commonly constructed by percussionists are the Turkish crescent (Jingling Johnny), which was scored for by composers ranging from Joseph Haydn to Hector Berlioz and even John Phillips Sousa, as well as the Mahler Hammer, which has a short but paramount role in Mahler's Symphony No. 6. in A minor. One might suggest integrating instrument and implement construction into the percussion curriculum so that individual students receive guidance during the process.

\footnotetext{
19 “Rzewski, Frederic,” IMSLP, accessed June 22, 2019, https://imslp.org/wiki/Category:Rzewski,_Frederic.

${ }^{20}$ Schick, 11.
} 
A piece that can serve to introduce instrument construction is Casey Cangelosi's Naglfar (2009). The piece calls for twenty chopsticks to be suspended via individual threads that are attached to a "thread anchor." While rapidly rattling a metal nail in the crevice of a wood block, the performer is to "clip the chop sticks one by one for approximately 40 seconds. The chop sticks should fall straight down onto the stage making a 'clacking' sound." The instructions lack detail in instrument construction and an infinite number of ways in which the student can construct the chopstick instrument result. For example, the percussionist can glue the string to the chopsticks, or they could drill a hole into the top of each stick and thread the string through, as per the common wind chime construction. Fortunately, the technique adopted will cause little or no consequence to the resulting sound or dramatic character of the passage. The responsibility of the performer to creatively problem solve and construct this simplistic instrument represents a necessary skill for the professional percussionist and is thereby a welcomed addition to the multiple percussion repertoire.

\section{Instrument Mounting}

Mounting of instruments is another craft that should be fully explored in the curriculum. Multiple percussion at its core may be perceived as the manipulation of conventional percussion performance practice, which applies to technique and instrument affixation in tandem. Dating back to Stravinsky's novel directions for the Histoire du Soldat percussionist to play the tambourine, strictly a handheld instrument at the time, "by mounting it on a cymbal stand and playing it with a mallet," this creative spirit has persisted within solo, chamber, and large ensemble contexts. $^{21}$

\footnotetext{
${ }^{21}$ Steven Brown, "Multi-Percussion Setup Design,” 36.
} 
Joe Tompkins's To Varèse (2013) presents advanced equipment and setup challenges within an explicitly predetermined instrumentation and a detailed setup diagram (Fig. 2.5). Firstly, unique aspects of the instrumentation may present issues of acquisition for the student, which includes a 28 " concert bass drum played with a soft foot pedal, Meinl foot cabasa, Brazilian tamborin (tamborim), and 16" floor tom. Students may be tempted to consider substitutions, in which case advanced problem-solving skills are needed to creatively determine how available instruments may fulfill various roles. Research is strongly recommended so that appropriate sound qualities can be achieved: a headed concert tambourine that uses muffling of both head and jingles will create a drier sound, a floor tom with the resonate head tuned higher to emulate the resonance of an African lion's roar, and a tamborin tuned to a high pitch per its cultural, Brazilian standards. The performer who proactively researches Varèse's Ionisation (1931) would also discover the appropriateness of orchestrating the snare drum on a deep military drum, a "Field or Parade Drum" according to Morris Goldenberg. ${ }^{22}$

${ }^{22}$ Edgard Varèse, Ionisation, in Modern School for Snare Drum ed. Morris Goldenberg and Anthony Cirone (Van Nuys, CA: Alfred Music, 1955/2002), 163. 


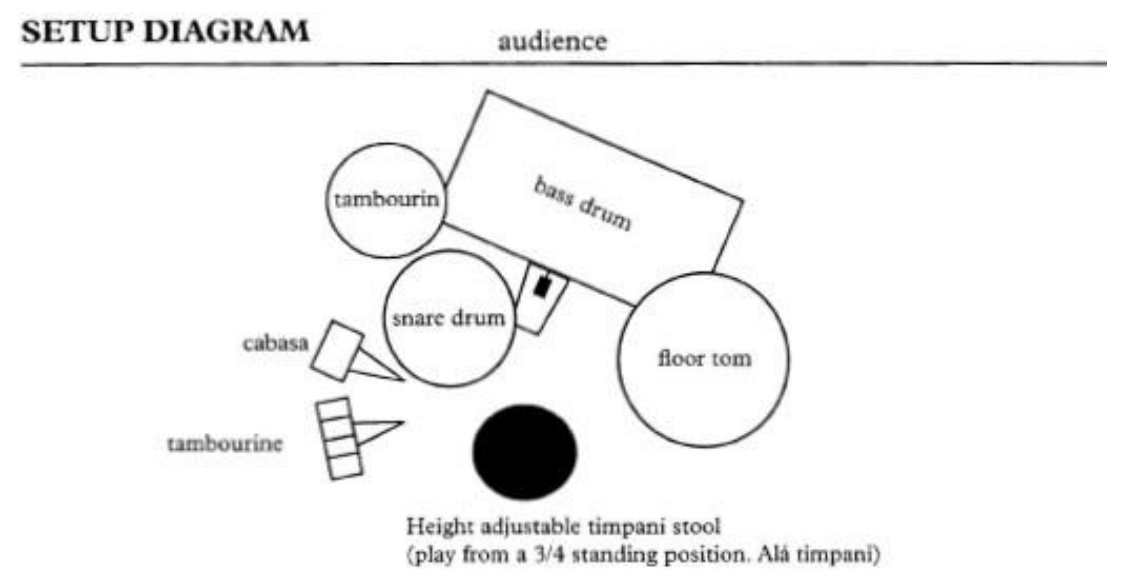

Fig. 2.5 To Varèse, Setup Diagram

In addition to the challenges in orchestration, the mounting of the chosen instruments in To Varèse presents further difficulty. The mounted tambourine and cabasa are unusual problems with little precedent. The tambourine could be effectively mounted on a cymbal stand with a boom that places the instrument in striking path of a typical foot pedal. In such a scenario, securement of the foot pedal must be addressed since it would not be fastened to a bass drum hoop, as per convention. Creating a similar sound via the means of the foot is equally challenging if a substitution is sought. To Varèse showcases the various obstacles in instrument mounting that are cornerstone to any professional percussionist. Interestingly, Nebjosa Zivkovic includes a photograph of his setup for CPAX: STRAH (1987), in affect revealing mounting and hardware solutions as well as instrument choice (Fig. 2.6) ${ }^{23}$ However, percussionists may need to find creative solutions in mounting with the hardware available to them. In addition, CPAX:STRAH continues to be more of an exception than a convention at present.

\footnotetext{
${ }^{23}$ Nebjosa Zivkovic, CPAX:STRAH (Celle: Moeck Verlag, 1987).
} 


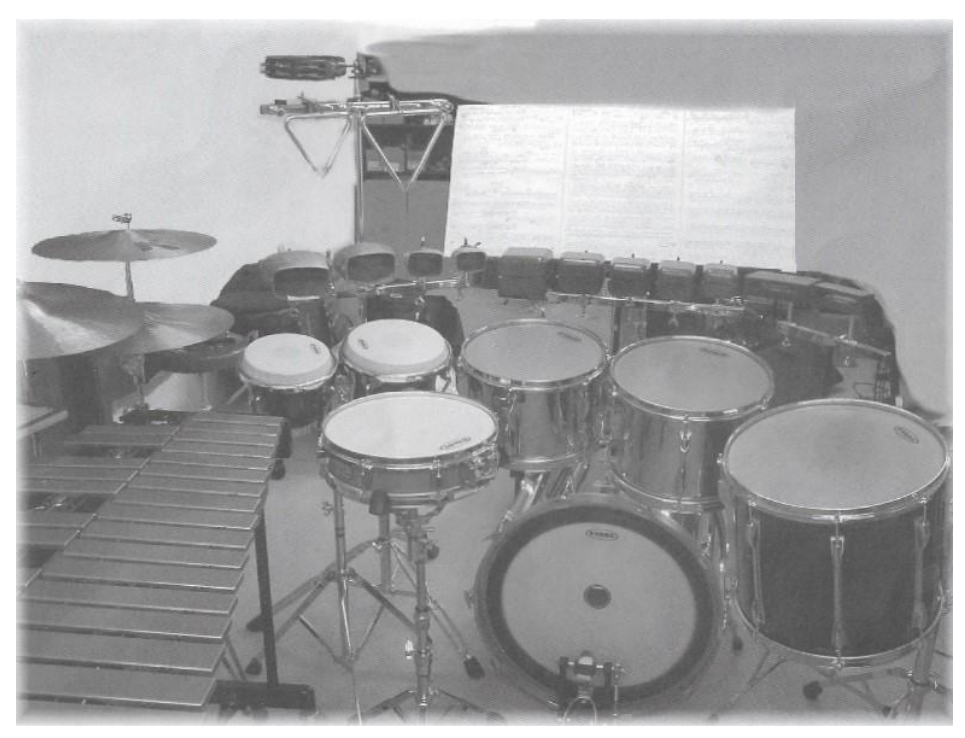

Fig. 2.6 CPAX:STRAH, suggested setup

\section{Instrument Placement}

The setup design encompasses the positioning of instruments in relationship to one another, as well as in relationship to the performer. The percussionist should be encouraged to consider instrument choice, manipulation, and mounting method simultaneously with setup design. Ideally, the percussionist would not feel restricted to a composer's diagram, but rather view it as a suggestion or "starting-off point." Instructors might encourage students to continue thinking critically and experiment with possible solutions. The duplication of an instrument might better portray its multiple roles, as in the cowbell for The Love of L'Histoire described above, or to better facilitate the percussionist's technical movement, which might present obvious deviations from the suggested setup diagram. While Petrella suggests caution when duplicating because of the inherent "timbral inconsistency" of any two instruments, I suggest the duplication technique regularly with my students, even if only for the sake of exercise and exploration in a non-performance situation. ${ }^{24}$

\footnotetext{
${ }^{24}$ Defining aspects of "timbral inconsistency" are from Petrella, The Multiple Percussion Book, 13.
} 
The logistic perspective of the setup design is simply instrument placement. The percussionist must be mindful that the acoustic properties of the instruments, as well as the relative ease and facility of the performer around the setup, are not ill-effected. Playing surfaces of the instruments should be on well-defined and level horizontal planes, allowing for playing between instruments with minimal variation of height. This may prove to be difficult when working with instruments of various depths, in which hardware must be chosen and positioned carefully. Using multiple trap tables might solve the problem of positioning sitting instruments of various depths. Convoluted foam of various thickness may be very beneficial as well, which can also be used to provide full resonance for found instruments that do not have mounting hardware.

Common mistakes in instrument placement should be avoided. Using music stands to secure instruments creates unwanted sympathetic vibrations as they are struck. Beginning students with positioning instruments higher than necessary causes excess tension while playing. Instruments should be positioned so that all the desired playing spots are easily accessible and without obstruction of other instruments, trap tables or music stands. Instruments should be angled appropriately in produce the desired resonance. Consideration of the position of the percussionist to avoid striking an instrument at a contracted position is necessary. For example, the tambourine is conventionally performed relatively flat to create a dry sound, which should be mimicked when mounting the instrument.

To avoid extraneous sound, instruments and pieces of hardware should not touch another. All hardware should be fashioned with the correct fasteners, cymbal sleeves and felts, rubber feet and bushings. Carefully placed pieces of moleskin may absorb some of the shock between instruments and hardware during performance. Making the instruments sound their best will not only make for a better performance or recording session, but will inspire the percussionist to 
practice and perform their best. When the instruments sound good, it is easier to listen to the sound intently and discover sound properties that can ultimately inspire a musical and expressive performance. In general, while many percussionists may perform the intermediate repertoire with adequacy, improper instrument placement will serve as a serious roadblock to the advanced repertoire.

\section{Setup Design}

The term setup design has been used in general to describe the previous equipment and setup facets in totality, but also as a description of instrument setup for indeterminate instrumentation, specifically. I chose to dissect the facets of instrument choice, manipulation, mounting and placement for the sake of clarity, which might illuminate the different skills and concepts for instructor and student. Factors such as available instruments, technical preferences, and acoustic properties of the performance space contribute to an individual's choice of equipment and setup. These facets do not transfer from one performer's realization to another, so percussionists should continue to think critically and explore solutions throughout their preparation of a piece of music.

In cases where a diagram is not provided, additional steps are required in the preparation of the setup design. Steven Brown suggests firstly making a complete instrument list, noting which "instruments are the most prominent throughout the piece so that you can place them in

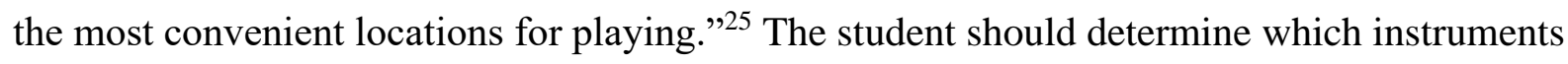
are sounded in quick succession and thereby beneficial to be near each other. After analysis of B of Xenakis' Rebonds (1987-89), the percussionist should recognize that there are many more instances where both hands play the drums simultaneously, while instances where both hands

\footnotetext{
${ }^{25}$ Brown, “A Study in Multi-Percussion Setup Design,” Percussive Notes 21, n. 2 (January 1983): 35.
} 
play the wood blocks are relatively isolated to specific sections where the drums do not sound. This seemingly modest discovery will influence one's setup design to facilitate accessibility to the drums that is optimal for both hands, while the wood blocks can be placed off to the side, to avoid compromised drum access.

If possible, placing instruments of similar material together will not only facilitate the percussionist's movement and accuracy, but will provide the audience with a clear link between movement and produced sound. Instruments that are only used for a section of a piece should be positioned in a relatively separate station; the degree of isolation determined by the amount of time to move. Composer Rolf Wallin illustrates two isolated "stations" of instruments in Stonewave (Fig. 2.7), a massive setup whose diagram only provides general guidance. Ideally, the advanced percussionist would conclude a similar setup without Wallin's suggested diagram. Fortunately, percussionists preparing Stonewave can save time and energy by setting up either Drums I or Drums II for an individual practice session and still be fully productive, because the two stations never sound simultaneously. An example without a suggested diagram is Xenakis' Psappha, which contains an entire instrument group (F) that does not enter until the piece's finale. The relative isolation of the group suggests they can be located in their own space, although the constant playing of Instrument $\mathrm{C}$ during the transition likely restricts its placement. 


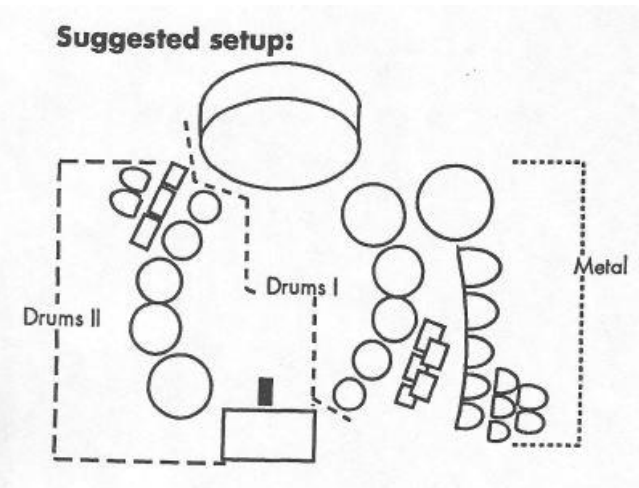

Fig. 2.7 Stonewave, suggested setup

While proximity of instruments is valuable for efficiency and accuracy objectives, Petrella states that "the aural aspect of multiple-percussion performance can be enhanced by the movement of the performer...slow, long-sustained sounds should be complemented by slow, long-sustained motions. ${ }^{" 26}$ In this way, placing longer sustaining instruments farther away will facilitate longer, larger gestures to match the sounds they produce. Since larger instruments typically produce longer resonances, it is logistically beneficial to place these instruments farther away as well. Smaller instruments should be positioned in the nucleus of the setup and closer to the performer, which will minimize extraneous space and grant further access and facility along more instruments. In his Percussive Arts article, Mark Berry offers three configurations for Maki Ishii's Thirteen Drums. ${ }^{27} \mathrm{He}$ coins his third and final configuration the "proximity-speed setup" because it offers the best facility because the performer does not have to reach over the larger drums (Fig. 2.8). ${ }^{28}$ Resonating cymbals and gongs would likely be placed farther from the performer as well, especially when a relatively imprecise playing spot is required to produce the

\footnotetext{
26 Petrella, 13.

${ }^{27}$ Mark Berry, “Thirteen Drums for Percussion Solo, op. 66: Interpreting in Concurrence with Make Ishii's 'SpaceTime' Concept," Percussion Notes 47, no. 5 (2009): 50-59.

${ }^{28}$ Ibid., 58-59.
} 
desired sound. In this way, sound properties and setup design directly contribute to the technique, musicality and expression in multiple percussion.

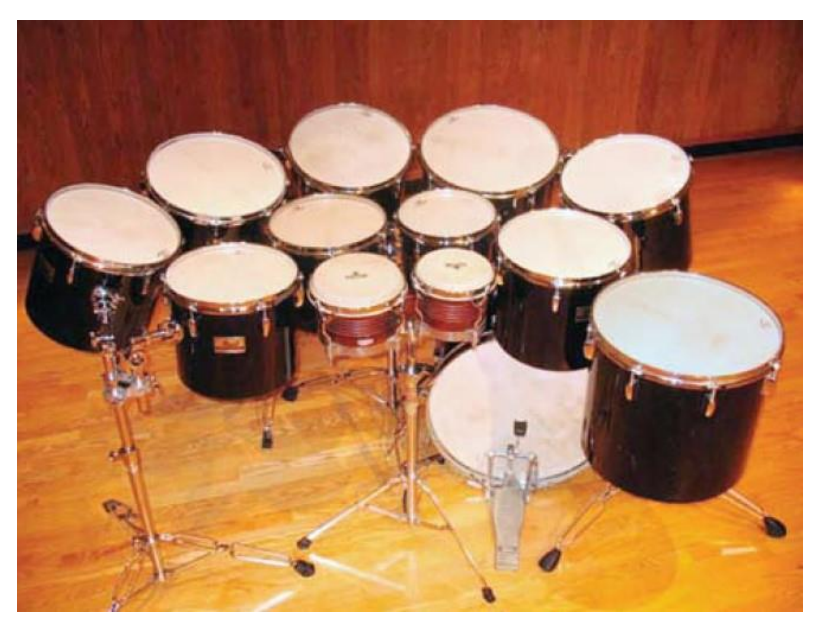

Fig. 2.8 Thirteen Drums, Berry "proximity-speed setup"

\section{Indeterminate Instrumentation}

Scores of an indeterminate style positions the equipment and setup dimension at the forefront. The multiple percussion solo medium has a long history of such indeterminacy; John Cage's 27'10.554" for solo percussion (1956, published 1960) calls for instruments in four groups: metal, wood, skin, and all others (e.g. electronic devices, radios, whistles etc.). Cage provides some suggestions in terms of instrument, but ultimately choices are left to the performer. He further instructs the performer's exploration of sound to be "exhaustive rather than conventional." ${ }^{29}$ Yet, to facilitate the virtuosic, dense passages, the instruments should be limited to what can be reasonably be situated around the performer. Wiggins offers various analytic perspectives of density, pitch range and timbre, ultimately suggesting that "wood, metal and skin sounds have to be placed close enough together to be struck in passages that flow through all of the instrument groupings in rapid succession." ${ }^{30}$ The necessity to traverse the setup might not

\footnotetext{
${ }^{29}$ John Cage, 27'10.554”' (New York City: C. F. Peters Corp., 1960), notes.

${ }^{30}$ Tracy Wiggins, "27'10.554" by John Cage and The King of Denmark by Morton Feldman and Their Influence Upon Thomas DeLio's as though" (DMA document: University of Hartford, 2009), 20.
} 
advocate for excessively large instruments in general. Instruments that provide quality sounds with the same implement will circumvent awkward implement changes.

The student should also become accustomed with choosing instruments that offer the widest breadth of possible sounds. Instruments that offer timbral variety from different playing spots, implements, or performance technique will maximize the palette within the limitations of the setup. Efficient choice of instrumentation is a necessary skill for modern dance accompanying as well, which is an increasingly common performance opportunity for percussionists. Much acclaim should be granted to the vibraphone because of its wide range of (a) pitch, via three octaves or more, (b) timbre, via a variety of appropriate implements and extended techniques, and (c) resonance, via its pedal and motor. Other instruments do not offer such a capacity, but the percussionist should develop this skill to maximize the potential of their multiple percussion setup. For example, choose a cymbal that can be fully activated quickly when struck on the edge with the shoulder of a stick, sounds light and articulate on the top of the cymbal with the bead, and provides a piercing percussive sound on its bell. Petrella notes that "set-ups comprised of instruments that provide a wide timbral diversity help the audience easily discern the different colors." 31 This suggests that the instruments should sound distinct (a) within each instrument group and (b) between the various groups (i.e., material).

Indeterminate instrumentation requires advanced problem-solving skills for a student seeking to realize Xenakis' Psappha. Of course, problems within the equipment and setup dimension are met with equally challenging issues of notation (described in Chapter 3), technique, and conceptual performance, which may explain the respect that percussionists have for this standard piece in the advanced repertoire. Bill Sallak effectively discusses instrument

\footnotetext{
${ }^{31}$ Petrella, 11.
} 
choice regarding Xenakis' implicit directions and intentions, while creating the "most efficient and comfortable configuration of instruments for ease of performance." 32 The piece is scored for seventeen instruments, grouped by material (skin or wood, metal) and register (high, medium, low), but the composer gives the performer more responsibility by describing accent possibilities, including orchestration on a different, but similar, instrument. However, through an analysis of select passages of the work, Sallak posits "more or less universal" requirements of the setup design, one example being to assign $\mathrm{C} 3$ to a pedal bass drum, which allows the percussionist to devote both hands to playing the three Group F instruments. ${ }^{33}$ Although the possibilities of instrumentation may seem endless at first, the student's informed recognition of implicit parameters, such as the technical demands of select passages, will inherently influence instrument choice.

By recognizing explicit and implicit directions, deliberately considering sound properties and skillfully crafting setup design, the percussion student can transcend into the advanced repertoire and thrive in the realization of works for indeterminate instrumentation. Details of advanced performance problems in the equipment and setup dimension and my solutions are provided under the case study of David Lang's The Anvil Chorus.

\section{David Lang, The Anvil Chorus (1991)}

Performed on April 8, 2017, D.M.A. Recital in Falbo Theater, West Virginia University. Scored for fourteen instruments, David Lang's The Anvil Chorus (1991) is notoriously challenging in terms of the equipment and setup. The piece has become a standard work in the multiple percussion repertoire since Steven Schick's premiere performance. Undoubtedly, the

\footnotetext{
${ }^{32}$ Bill Sallak, "Informed Indeterminacy: Guidelines for Instrument Choice in Iannis Xenakis' 'Psappha'," Percussive Notes 40, no. 2 (April 2002): 55.

${ }^{33}$ Ibid., 57-58.
} 
challenges of the equipment and setup, as well as the individuality of one's creative solutions, contribute to the appeal the piece has for performers and audiences alike. Schick describes the rhythmic cycles in the piece as being "eminently audible...[but] a potential problem lies in the instrumentation." 34 This suggests the significant role that instrumentation holds for an effective portrayal of the piece's structures. Despite the individualized nature of one's chosen setup design for the piece, understanding equipment and setup concepts that govern its realization will ultimately lead to a more successful performance of this piece and develop a student's fluency in the equipment and setup dimension. After attempting many different strategies of instrument choice, mounting and placement, I finally decided on the setup design for performance seen in Fig. 2.9.

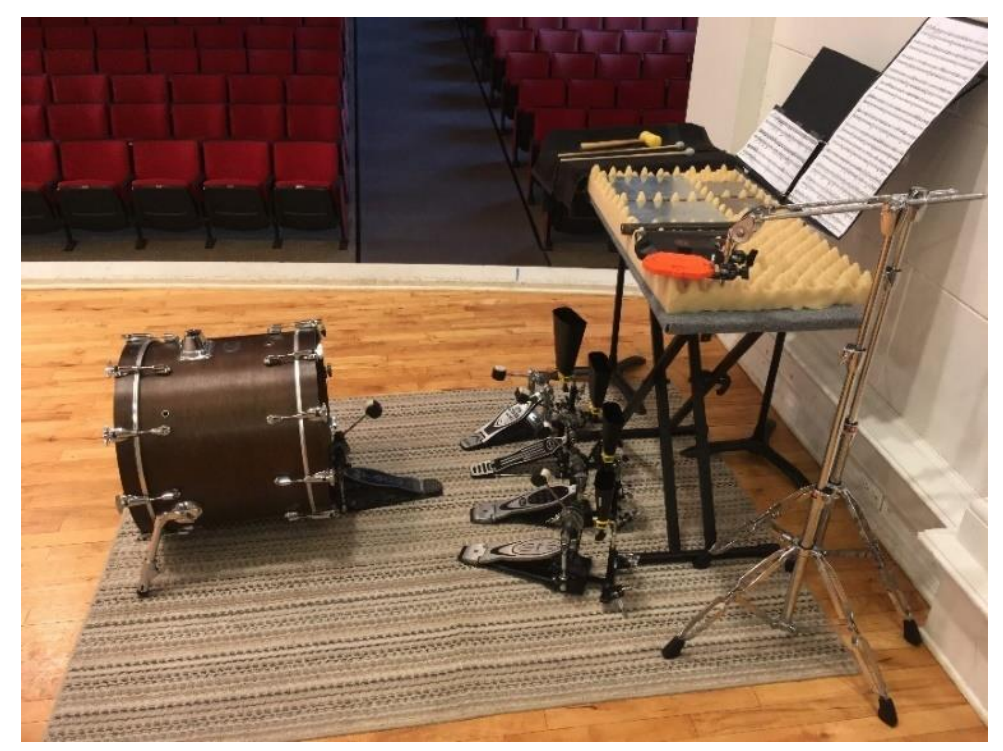

Fig. 2.9 The Anvil Chorus, Greco setup

In the instrument key at the beginning of the score, Lang provides short descriptors for the instrument groups: three resonant metals, four non-resonant metals, four non-resonant metals operated with foot pedals, one bass drum operated with a foot pedal, and two wood blocks (Fig.

\footnotetext{
${ }^{34}$ Schick, 27.
} 
2.10). The composer does not offer a suggested setup diagram for the percussionist, but a current trend is to follow Schick's description in The Percussionist's Art, an "oral performance tradition" of the work molded by conversations with the composer rather than explicit directions from the actual score. ${ }^{35}$ While the score articulates the instrument groups only via the amount of resonance (as well as the technique of hand versus foot pedals), the composer orally communicated to Schick that relative clarity of pitch should also be considered. This implies that the resonant metals not only have a longer sustain, but also a clearer pitch. Likewise, the nonresonant metals should produce a distorted noise with a less clear pitch, in addition to a shorter duration.

INSTRUMENTS REQUIRED:

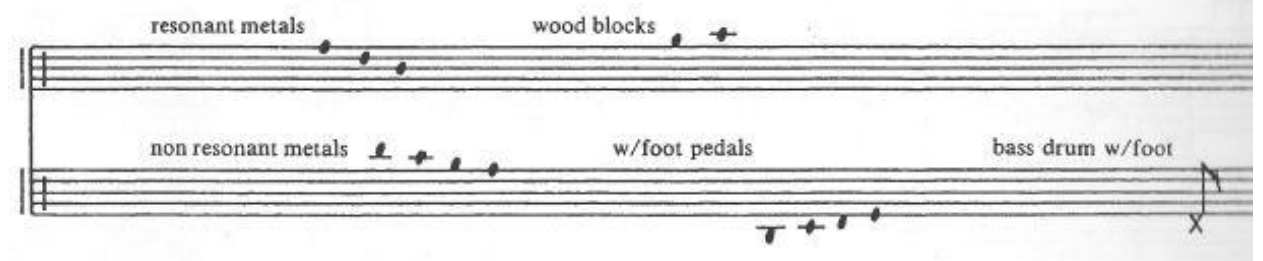

Fig. 2.10 The Anvil Chorus, instrument key

Along with addressing each instrument individually, attention should be paid to the groupings of instruments and the relationship of sounds between them. Schick describes three possible models structuring instrument groups: atomized, unified and modular. ${ }^{36}$ While Schick describes these models within the lens of Psappha by Iannis Xenakis, the concepts apply to The Anvil Chorus and any other multiple percussion work. An atomized model would use instruments of maximum distinction, regardless of the composer's suggested groupings. A unified model would contain coherence within each instrument group via a similar timbre. Furthermore, at least

${ }^{35}$ Ibid., 31.

${ }^{36}$ Ibid., 194-196. 
one relationship (intervallic, duration, tone clarity, etc.) would match all the different instrument groups, thereby forming an element of unification between them. Like the unified strategy, a modular module would also contain groups of instruments that "are maximally distinguished from each other via instrumental color." ${ }^{37}$ However, in this case, each group would have different intervallic, duration, and tone clarity relationships.

For The Anvil Chorus, one may choose to stock the resonant metal group with instruments that produce recognizable, consonant intervals. This would help emphasize the clarity of pitch and resonance of the group by inferring tonality. I chose to use found pipes that had a relatively equal definition of pitch and that created a root position chord, providing maximum clarity of tonal structure and a feeling of resolution as early as measure 2 . Performers can even fabricate sixxen (tuned metal pipes), as per Brett Reed's instructions, described in the context of Xenakis' Pleiades, to create the intervallic relationships they desire. With uniformity within the resonant group in place, I consequently chose to eliminate the atomized model.

A highly unified model would match the relationships of intervals, tone clarity, and resonance between instruments in all groups, thereby maximizing the intra- and interrelationships. I found that the modular model offered more timbral differentiation and interest, since eleven of the fourteen instruments are metal. I chose to orchestrate opposing relationships between the resonant and the non-resonant metal groups, using steel plates for the latter. The steel plates became thinner as they got larger, thereby increasing in distortion and shortening in duration as the instruments descended in pitch. Despite blurriness of the tone, the highest pitched steel plate resembled tonic and seemed the most resolute in the group. Coherence of the foot pedal group will be addressed within the confines of instrument mounting.

\footnotetext{
${ }^{37}$ Ibid., 196.
} 
Logistic feasibility inherently governs instrument choices in The Anvil Chorus. Since all instrument groups in sound simultaneously for much of the piece, they must all be reachable from one location. Among those played with hands (i.e., the resonant metals, non-resonant metals, and wood blocks), the rapid movement between instruments within the same group requires them to be within close proximity and at the same height (Fig. 2.11). Schick orchestrates the non-resonant metals on brake drums, which are common instruments in the percussion studio and generally produce a desirable sound for this situation. However, brake drums generate of number of performance issues: (a) a precise playing spot is required, (b) their open centers might trap a mallet during performance, and (c) an unnecessarily large amount of space is needed, due to their shape. Instead, I chose to use metal plates that produce a relatively uniform timbre regardless of where they are struck. They also take up less space and are easily moved (Fig. 2.12).

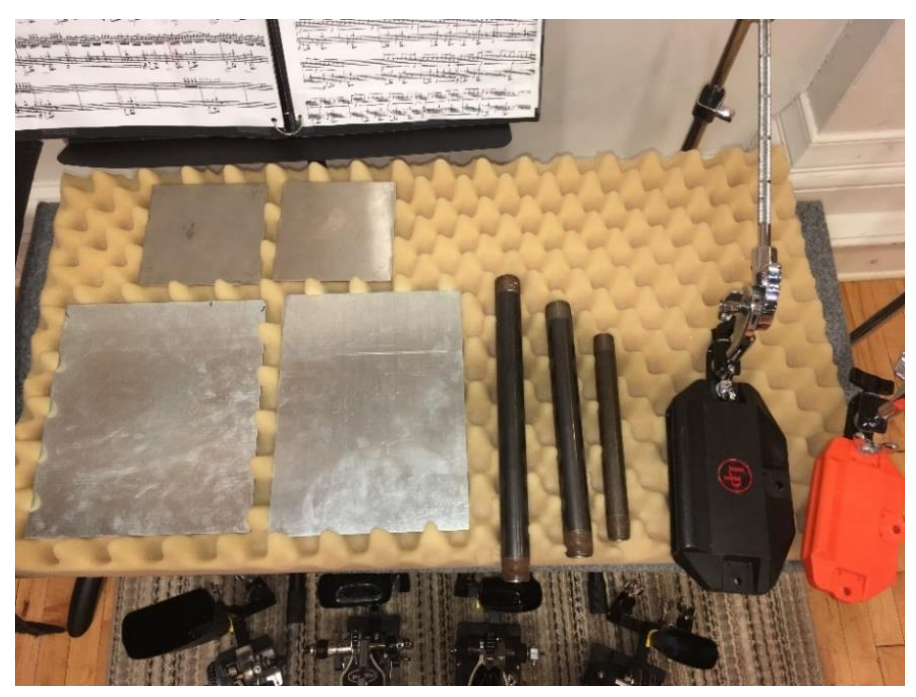

Fig. 2.11 The Anvil Chorus, Greco setup for instruments struck with handheld mallets 


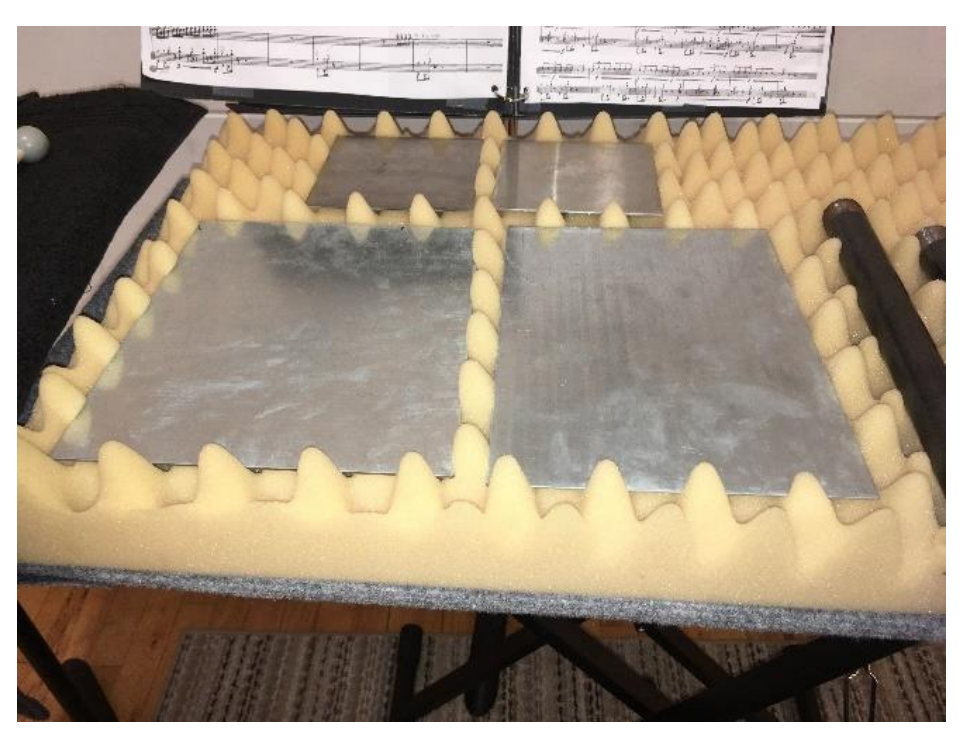

Fig. 2.12 The Anvil Chorus, Greco non-resonant steel plates

Since each hand is isolated to one group for much of the work, with no clear melodic lines crossing over from one instrument group to another, the performer need not worry about matching implements. The composer indicates using chime hammers, a difficult challenge to effectively incorporate into the entire work. However, chime mallets can make for an interesting timbral contrast and a dramatic visual portrayal of the blacksmith hammering that inspired the composition. I chose to use a chime hammer on the non-resonant metals from the beginning to m.155, producing a full sound when the hammer strikes the surface at a slight angle. I used the three beats of rest to switch to a hard rubber mallet for mm. 156-199, a necessary change to facilitate the fast rhythms and low dynamic at the beginning of the section. To help associate the recapitulation of previously heard themes and for dramatic effect in the final section, I chose to switch back to the chime hammer from "suddenly a lot slower" (measure 200) to the end. To coordinate the mallet change, I choreographed the switch after the first strike in "suddenly a lot slower" (m. 200) was made with the hard rubber mallet. 


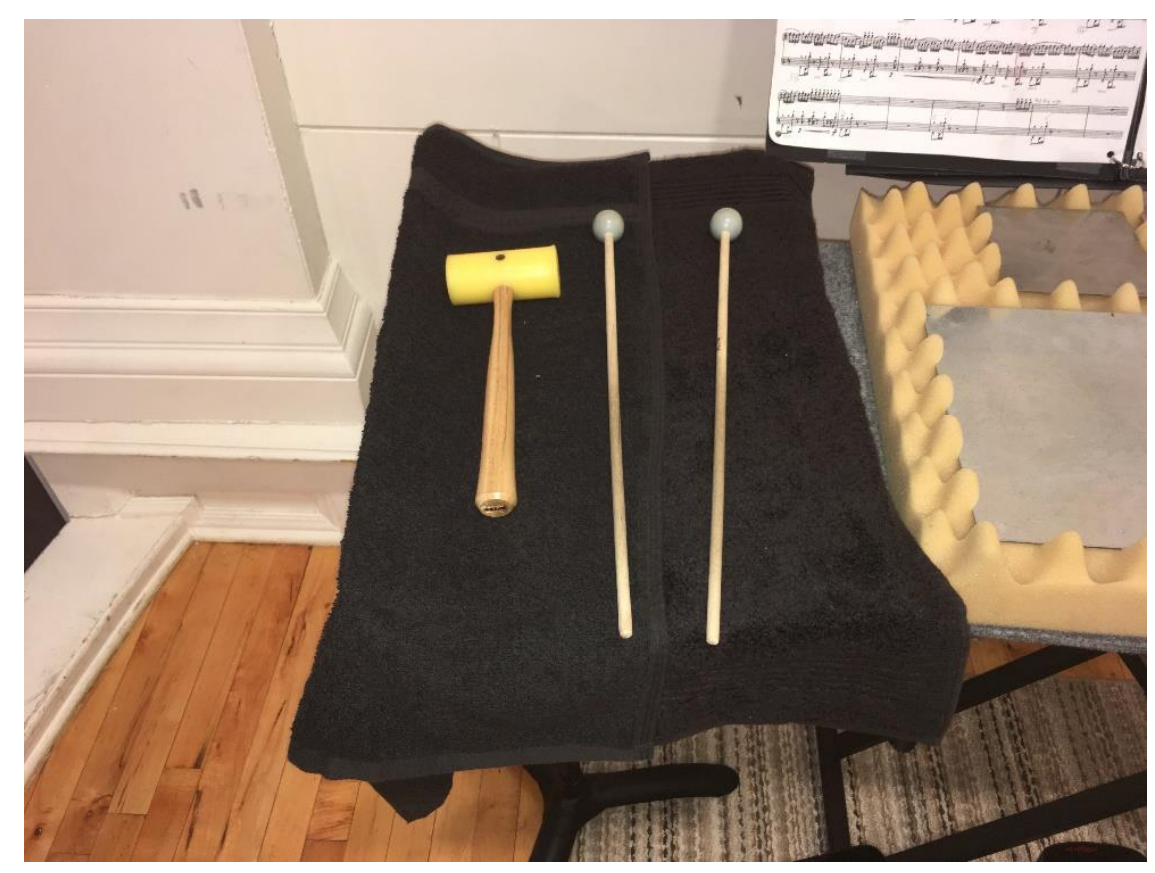

Fig. 2.13 The Anvil Chorus, Greco stick tray

The implement changes required that a stick tray, in this case a music stand, be placed directly to the left of the trap table holding the instruments (Fig. 2.13). Since a small toss was necessary to execute the fast switch of implements, the stick tray must be carefully prepared. A carpet square placed on top served to muffle the landing sound, and the lip of the music stand situated on the left side operated as a failsafe to block a rogue mallet. Two black towels were placed on top of the stick tray in such a way that the overlap occurred on the left half, which was the target zone of the toss, to help soften the mallets' landing.

The resonant and non-resonant metal instruments were placed on the same piece of convoluted foam. The peaks of the foam held the pipes in place and appropriately separated them from coming in contact with another. The four metal plates were placed in two rows to facilitate easy movement between them (Fig. 2.12). I found that the plates were not stable when struck with the chime hammer when they were simply placed on top of the foam, so I cut peaks of the convoluted foam where each plate would rest to form a mold. Full peaks were left in between the 
plates, thereby restricting any shifting of their position and to act as boundaries. Since the foam peaks were cut, the metal plates rested a little lower than the resonant pipes, which proved no consequence provided the isolation of the groups to separate hands throughout the piece.

Although conventional percussion instruments, the wood blocks also demand further consideration for any realization of the piece. The rapid sixteenth-note succession between the wood blocks and the resonant metals in the same hand (mm. 135-147) suggests that the two instrument groups must be placed in very close proximity. The passage is technically much easier to play if both groups rest at the same height. I experimented with using convoluted foam pieces of different depths to level the playing surface of traditional wood blocks. I also observed a trend in current YouTube recordings of percussionists using slabs of wood but found they did not provide the volume to possibly balance with the metal instruments. I believe that amplifying the slabs would be counter to the physical character of the piece.

Ultimately, I orchestrated the wood blocks on LP Jam Blocks. The instruments were easily mounted onto boom cymbal stands (Fig. 2.14), which allowed full control of their positioning in terms of both height and angle. The virtual indestructibility of the jam blocks was another benefit, especially since I used the same mallet to strike the resonant metals. The jam blocks successfully produced a "woody" timbre that was very distinct from the other instruments. However, the very precise playing spot required to produce a quality, consistent sound certainly posed a particular technical challenge. 


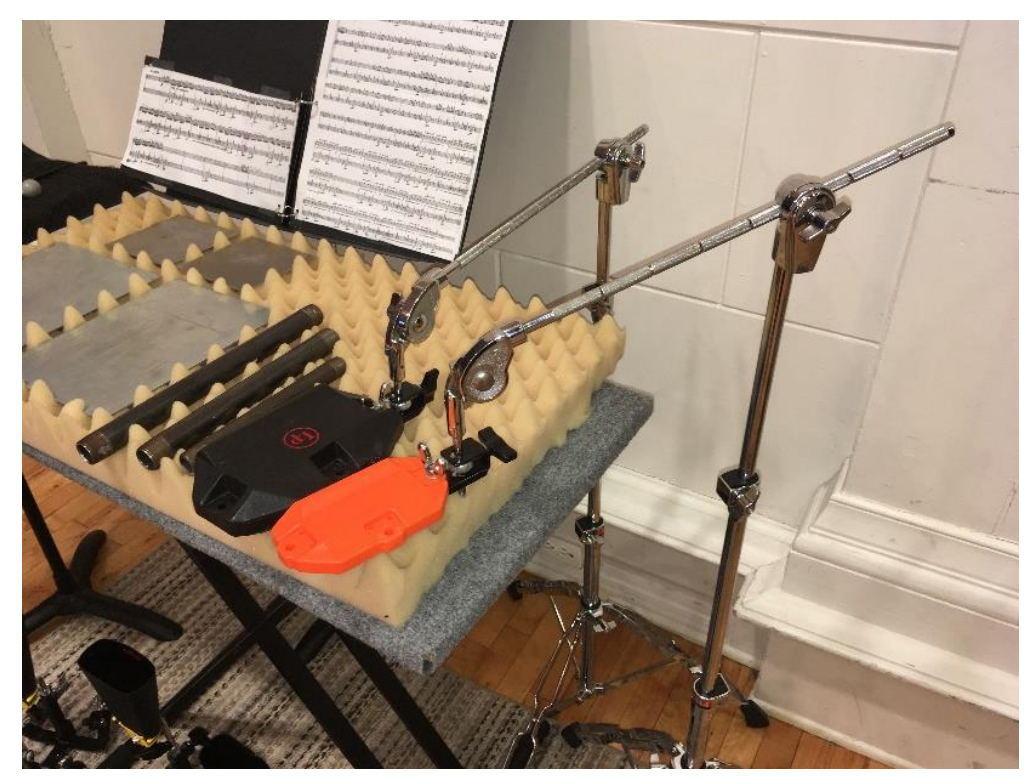

Fig. 2.14 The Anvil Chorus, Greco setup of wood blocks

Instrument design, mounting and placement of the foot pedal operated instruments are unprecedented challenges in The Anvil Chorus. Simply acquiring access to five foot pedals for regular practicing presents a daunting task for students and percussion studios. If the musician can purchase their own, Coleman describes techniques to utilize foot pedals to their full potential, such as cam adjustments that can produce the optimal angle and motion from the beaters. ${ }^{38}$ Coleman creatively adjusts his cam mechanism to strike Chinese opera gongs, a choice for the non-resonant foot pedal instrument group that he craftily mounts horizontally. He describes the opera gongs as "a good choice because of their small size, abrasive sound, and brief resonance." 39

The necessary cam and other foot pedal adjustments may be avoided by choosing instruments that can be mounted perpendicular to the ground, such as a tam-tam taped to the batter head of a bass drum, à la Schick's design. ${ }^{40}$ The taping appropriately muffles the tam-tam

\footnotetext{
${ }^{38}$ Coleman, "Instrument Design," 27-29.

${ }^{39}$ Ibid., 25.

${ }^{40}$ Schick, 30.
} 
and restricts any unwanted swinging, and the bass drum hoop allows for securement of the foot pedal. As Schick orchestrates two foot pedal metals in this manner, this technique requires the use of three bass drums, a luxury I did not have while preparing this piece. For logistic and musical purposes, I chose to orchestrate the foot pedal metals on cowbells.

Many performers have forgone total uniformity within the foot pedal instrument group, instead opting for a mismatch collection of distorted sounds. Notable performances available on YouTube in this realm include Matt Keown (cowbell, stainless steel bowl, frying pan, and hihat stack) and Evan Chapman (cowbell, ribbon crasher, trashy stack of cymbals, and large metal bin). ${ }^{41}$ However, I sought to match the relative intervallic relationships of the non-resonant metals (steel plates) with the non-resonant foot pedal group, (Fig. 2.15), thereby choosing to apply the uniform model to these two groups.

When considering establishing a sense of cohesion, Schick describes how the "mode of performance... allow[s] for the development of a strong sense of internal coherence among an otherwise set of objects." 42 Therefore, since the cowbells are the only metals played with foot pedals, there is an inherent level of coherence in those instruments, regardless of instrument choice. The cowbells took up little room and were also easily mounted using foot pedal attachments. In addition to the practical advantages, the cowbells also met Coleman's criteria of producing an abrasive sound with a short resonance. Varying amounts of moleskin were applied to the instruments, a relatively easy procedure that helped muffle the resonance.

\footnotetext{
${ }^{41}$ Matt Keown, “The Anvil Chorus, by David Lang,” uploaded by Vic Firth, YouTube, March 9, 2017, https://www.youtube.com/watch?v=_DUFzprscPk.

Evan Chapman, “The Anvil Chorus by David Lang (Multiple Percussion)," December 11, 2012, YouTube, https://www.youtube.com/watch?v=2y-B6QMMmCw.

${ }^{42}$ Schick, 17.
} 


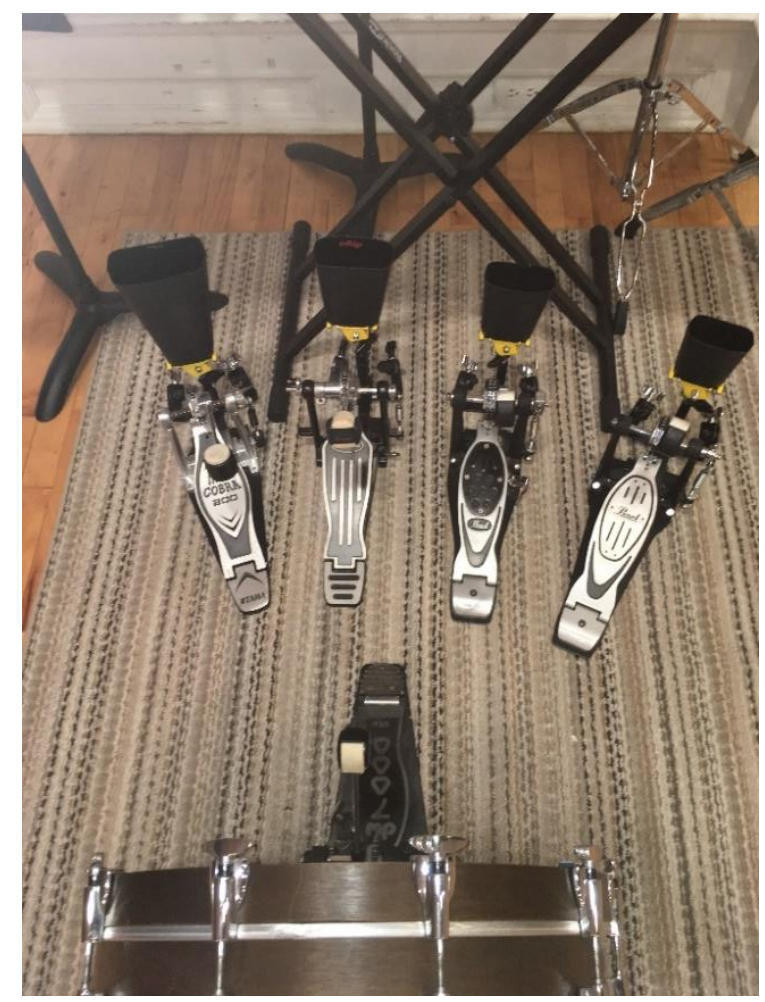

Fig. 2.15 The Anvil Chorus, Greco setup foot pedal cowbells

By fashioning instruments to have very short sounds, isolating the attack transients, the perceived difference between the instruments is diluted. The timbre of a sound derives primarily from its resonance, so muffling (i.e., moleskin etc.) is completely appropriate to produce articulate, crisp sounds of the foot pedal group that should "propel the work rhythmically and indeed even melodically." 43 In this way, a mismatched model that foregoes uniformity within the foot pedal group, although not my ultimate design for the piece, can be appropriate. However, particular attention should be paid to restrict the resonance, thereby isolating the attack transient frequencies so sound can also contribute to adhesion within the group, not just the performance technique of the foot pedal.

\footnotetext{
${ }^{43}$ Ibid., 31.
} 
The placement of the foot pedals directly affected my preparation of The Anvil Chorus, which was interconnected with the decision to sit or stand to play the piece. Schick describes that Lang "prefers that the performer stand while playing," but challenges emerge from such a position. ${ }^{44}$ For example, I found that the bass drum had to be moved behind me. Consequently, the pedal was then operated by the heel of my foot. This presented a technical learning curve, I even broke one footboard in half. However, I eventually gained a sensitivity and comfortability for the technique, which ended up being a rather idiomatic way of operating the pedal. The position also facilitated the quick interplay of bass drum and the foot pedal metals by shifting my weight from foot to foot. Moving between the foot pedals is inherently slower from a standing position than the sitting position. I also found balancing while standing on one foot pedal, or even two foot pedals, to be tricky and a bit unnerving. Stickings and movement of the feet needed to be carefully choreographed and strictly followed to maintain proper balance and poise.

The pedals needed to be precisely positioned so that both feet could access the inside two non-resonant and the bass drum pedals. I found that that left end of the bass drum pedal must be aligned with the right side of the second metal foot pedal, and 2-3 inches needed to separate the ends of both foot pedals (Fig. 2.16). The placement was crucial so that I could share responsibilities between both feet, create easier stickings within the feet, and better maintain balance, posture, and poise throughout the piece. The firmness of the beaters needed to be graded in order to produce the best sound on their corresponding cowbells, and the distance between where the beaters rested and the instrument surface needed to be sequentially smaller as the cowbell rose in pitch.

\footnotetext{
${ }^{44}$ Ibid., 28.
} 


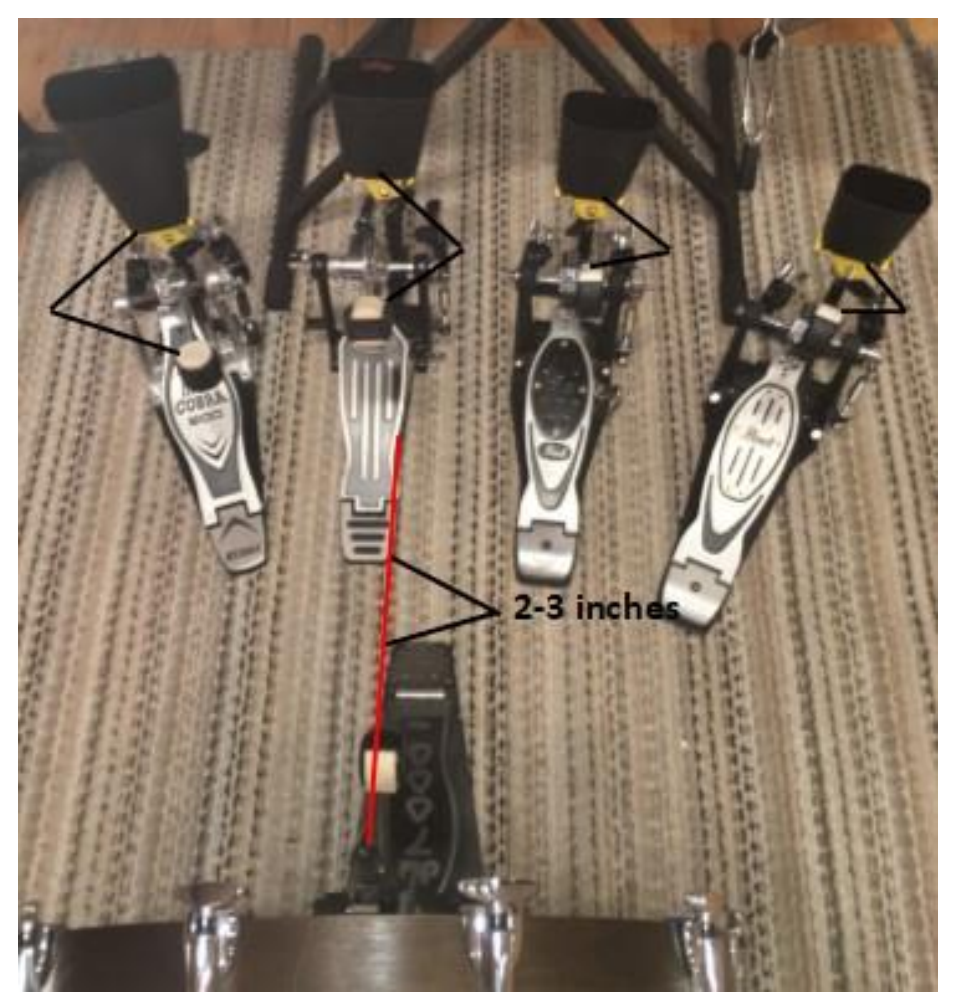

Fig. 2.16 The Anvil Chorus, Greco foot pedals with marks

Sitting for the piece would have allowed for easier, quicker movement between the pedals. However, the bass drum would have needed to be in front of the performer, thereby expanding the physical territory covered by the feet along the $x$ axis. Unless duplicated, the bass drum would most likely have needed to be off to one side of the setup, regulating all of its notes being played with one foot. My decision to stand was also influenced by a hindered facility while in the sitting position, losing a full range of motion to twist, lean, and reach in the upper body without my feet being grounded.

The securement of the foot pedals is necessary to avoid gradual shifting of the hardware during playing. Described above, Schick creatively addressed this problem by securely attaching the pedals to the bass drum hoops, but that also adds to the already extensive equipment demands. If a player chooses an instrument that is suspended independently of the foot pedal, such as a cymbal or cymbal stack, then securing the foot pedal in place presents an issue. Simply 
taping the pedal footboards to the floor with duct tape is a great solution if the equipment and the practice space are not needed for other purposes. A more conspicuous option might be to attach Velcro or other adhesive strips to the bottom of the footboard, where the strips could stay on the floor after tear down, serving to help place the instruments for future practice sessions. Another option is to clamp multiple foot pedals onto a single narrow strip of wood; the pedals gaining traction and sturdiness from each other. I found that the foot pedal mounts that held the cowbells offered ample sturdiness and ease of set up and tear down for every practice session. Using an oversized rug provided a space for all the instruments to rest and created a sturdy setup that was void of excess noise (Fig. 2.9).

The equipment and setup dimension require critical thinking and creative problem solving for the solo multiple percussionist. Facets of this dimension offer skills and concepts that are required in other percussive mediums and are likely to transfer to various contexts. Instrument choice, manipulation, mounting, placement, and setup design all presented challenges in my realization of The Anvil Chorus. I overcame these obstacles when I applied my experience from other solo and ensemble pieces, imagination, and attention to detail. It is important to remember that the equipment and setup may change throughout the learning and finalization phases of a piece and, in the case of advanced repertoire, potentially after numerous performances. With an informed perspective, one's procured skills will eliminate time wasted with avoidable tasks such as making ill-advised instrument choices and fixing improperly secured instruments. Interested readers are directed to Appendix A for my proposed graduate curriculum that develops skills and concepts within the equipment and setup dimension. 


\section{CHAPTER 3: NOTATIONAL STYLE}

Although not entirely unique to the medium, the notational method prescribed by the composer is a significant challenge for the solo multiple percussionist. Each piece that a percussionist encounters is likely to have a new notational system, mirroring the individuality of their instrument setup. The percussionist should seek to gain fluency in the composer's vocabulary and instructions, achieved firstly by remembering how the musical score relates back to the key legend and other performance notes. Following the Bloom taxonomies, percussionists should subsequently seek to understand the musical score, a primarily mental process that manifests in audiation of the musical score in one's inner ear. ${ }^{45}$ Applying the information refers to the transference of the musical score into the action of playing. A performer should then seek to analyze the parameters of the piece, including those under the control of the composer versus those at the discretion of a performer, a necessary step to formulate an interpretation. The instructor should encourage the student's ability to evaluate this information, which best occurs when the percussionist has experience with a wide survey of the literature. To create is the ultimate objective, which can be applied to the performance of any notated music, but emphasized in graphic, spatial, and text notation, since the performer acts significantly as partcomposer.

Solo multiple percussion music can be classified into two principal styles of notation: (a) modifications of standard, conventional notation or (b) unique, unconventional notations. Unconventional notational styles incorporate such elements as graphic, spatial, and textual devices. Each work in the repertoire has some degree of individualism in its notation based on its

\footnotetext{
${ }^{45}$ Laurie Richlin, Blueprint for Learning: Constructing College Courses to Facilitate, Assess, and Document Learning (Sterling, VA: Stylus Publishing, 2006), 45 - 51.
} 
significant parameters such as timbre, dynamic, rhythm, and pitch; even those following standard conventions employ key legends that may only apply to that piece's specific instrumentation. Nevertheless, since general stylistic features can be traced, concepts of interpretation can be learned, and skills can be developed that transfer from one piece to another, a curriculum can ultimately be theorized (Appendix B). A well-rounded percussionist should have experience in each of the notational styles; not only the various styles called on in realistic professional situations in large-, mixed chamber- and percussion ensemble literature, but experiential learning in a safe environment may build an appreciation for the art form and develop problem-solving and critical thinking skills.

While an overview of its history and available resources will help position the modern percussionist, a comprehensive survey of twentieth century composers' notational innovations and the various perspectives of the scholarly research is well beyond the parameters of this study. Interested readers should refer to resources from Schick and the edited collection by Kevin Lewis and Gustavo Aguilar for a more comprehensive history on how early composers of multiple percussion addressed notation. ${ }^{46}$ Despite issues of clarity resulting from the handwritten score, Stravinsky's line-staff notational style for Histoire du Soldat is remarkably readable. Similarly, although John Cage's 27’10.554” likely lacks any semblance to a musical composition at first glance, the score becomes quite intuitive upon an understanding of its structures. These early works for multiple percussion suggest that composers' intuition paralleled their ingenuity within the notational realm.

\footnotetext{
${ }^{46}$ Schick, The Percussionist's Art. Kevin Lewis and Gustavo Aguilar (eds.), The Modern Percussion Revolution: Journeys of the Progressive Artist (New York: Routledge, 2014).
} 


\section{Standard Notation}

From a pedagogical perspective, I hypothesize that percussionists should seek fluency in standard notation first, before progressing to unconventional notation devices. A pragmatic reason for such a curriculum is that percussionists' time and attention also address the other three dimensions simultaneously: equipment and setup, technique, and conceptual performance. Another benefit of firstly building standard notation fluency is the depth of representative repertoire that supply a vast amount of variation, many of which account for complex and mature pieces in the repertoire. Since standard notation repertoire spans all difficulty levels, it can be used to challenge the percussionist regardless of skill level in the other dimensions. Lastly, the use of standard notation in ensemble literature at large suggests its fluency for success in the professional realm.

The foremost convention for standard notation lies in the treatment of rhythm. The standard rhythmic notation allows the percussionist to quickly understand note speed and note durations, particularly with how those durations relate to one another. DeLancey articulates instructions through typical symbols in note length, meter, and tempo (Fig. 3.1). He also incorporates conventional symbols apart from the rhythmic components, such as dynamics and mallet type to be used at specific moments.

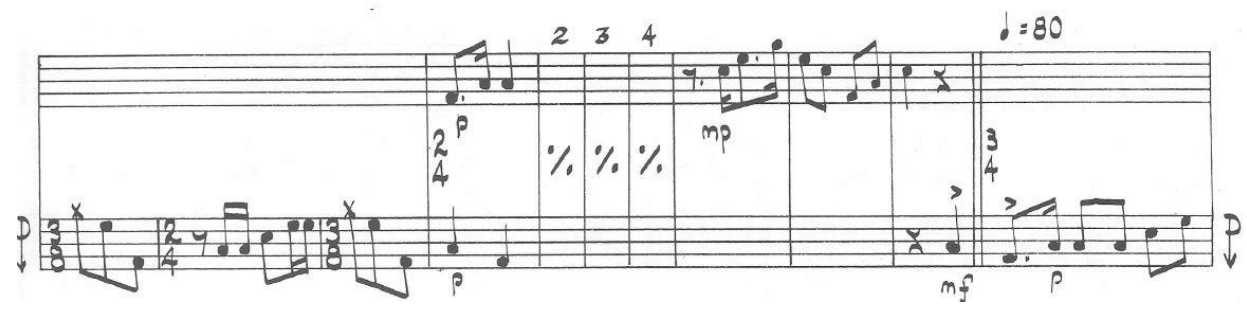

Fig 3.1 The Love of L'Histoire, standard notation

Composers have developed ways to creatively incorporate standard rhythmic values while manipulating other devices to individual taste. Udow and Watts omit the time signature for 
etude XII, a seemingly trivial change that grants the performer freedom to decide how note durations relate to a beat, if at all (Fig. 3.2). The lack of bar lines in the piece furthers the performer's choices concerning metrical hierarchy. Since there are no intrinsic accents provided, the performer must rely on phrase groupings and the agogic accents of longer notes to bring musicality to the work. The wide range of tempi indicated further influences the numerous interpretations that are possible for XII, despite its use of conventional rhythmic note values.

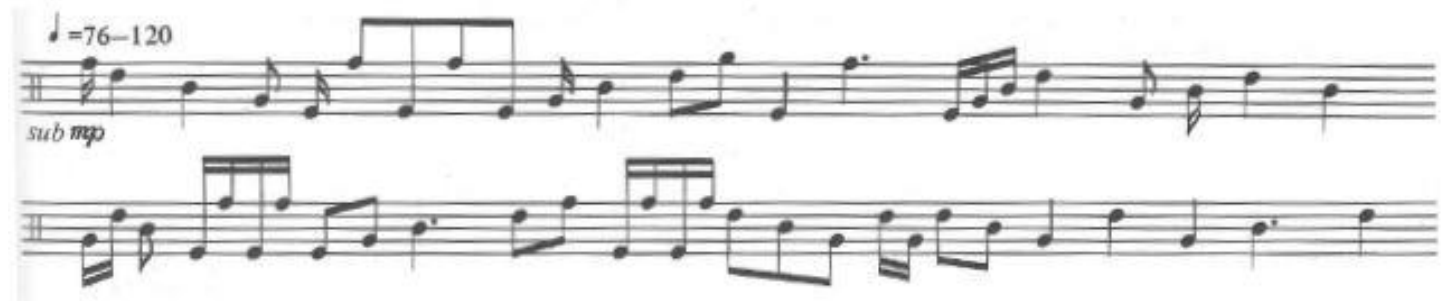

Fig. 3.2 XII, standard notation without time signature and bar lines

The "sliding scale" of standard conventions in modern notations, representative by the rhythmic treatment of XII, led Julian-Jones to conclude that "numerous inconsistencies in the designations of these notational systems...[have] presented challenges in clearly classifying notational systems." Despite the difficulties of classification, works with individual variations of standard notation can be opportune for building learning outcomes specific to the student. For example, incorporating pieces that maintain as many standard notation conventions as possible may serve to help reinforce sight-reading, music theory and ear training skills for students struggling in those areas. Contrastingly, repertoire with standard notation conventions can be used for students that excel in music theory, but perhaps need more development of skills with the equipment and setup, technique, or conceptual performance dimension of percussion. Within the realm of notational fluency, repertoire with standard notation conventions can also provide the student with a known base in which notational variations can be introduced. 
In addition to rhythmic instructions along the horizontal $x$ axis of a musical score, the unique instrumentation of multiple percussion works generates an inherent divergence in treatment along the vertical $y$ axis. Students that firstly build on their familiarity with the fiveline stave will (a) reinforce musical skills and concepts from other contexts and (b) have a stronger knowledge base. With a stronger foundation, the student will likely have more confidence in their progress and performance, as well as be more apt to notice details in notational variation and detail.

However, the five-line stave poses significant reading challenges when applied to nonpitched percussion. Theoretically, the five-line stave is best utilized for a piece of eleven instruments, occupying the lines and spaces fully without the use of ledger lines. Pieces that use less than eleven thereby occupy an inappropriate amount of space, at the very least, which may make navigating the score quite laboring. Similarly, pieces with tremendous instrumentation make deciphering the excessive ledger lines difficult. Aside from the appropriateness in five-line scale usage, Cook recognizes that "for years, the standard five-line staff has sufficed for a great amount of percussion music," making its fluency absolutely critical. ${ }^{47}$

The convention of pitch traditionally applied to a five-line stave, in which notes positioned higher in the stave indicate a higher pitch and vice versa, often do not apply to solo multiple percussion. The instruments of varying timbres typically do not have a clear continuum of pitch, making reading its rendering on five-line stave a difficult task. Beginner and intermediate pieces should be chosen that use either the lines or the spaces. A next logical step in the progression might incorporate both lines and spaces, each of which are regulated to a similar timbre. The similar timbre will help emphasize the melodic relationship between the instruments

\footnotetext{
${ }^{47}$ Gary Cook, Teaching Percussion with DVD, $3^{\text {rd }}$ ed. (Belmont, CA: Thomas Schirmer, 2006), 80.
} 
in that group, making a direct correlation between the notation, instrumental setup and sound. In the intermediate-advanced repertoire, DeLancey idiomatically positions two different five-line staves with unique clefs that indicate wood and drum groups for Love of L'Histoire (Fig. 3.3). The creation of two staves allows for easy deciphering of the notated instruments, while the separation of the two primary timbres allows for a perception of pitch between instruments that share the same timbre. For example, the performer is not lured to perceive the pitch relationship between a wood instrument and a drum instrument in this notation, which is a helpful strategy to negotiate a rather large setup for an intermediate-advanced piece.

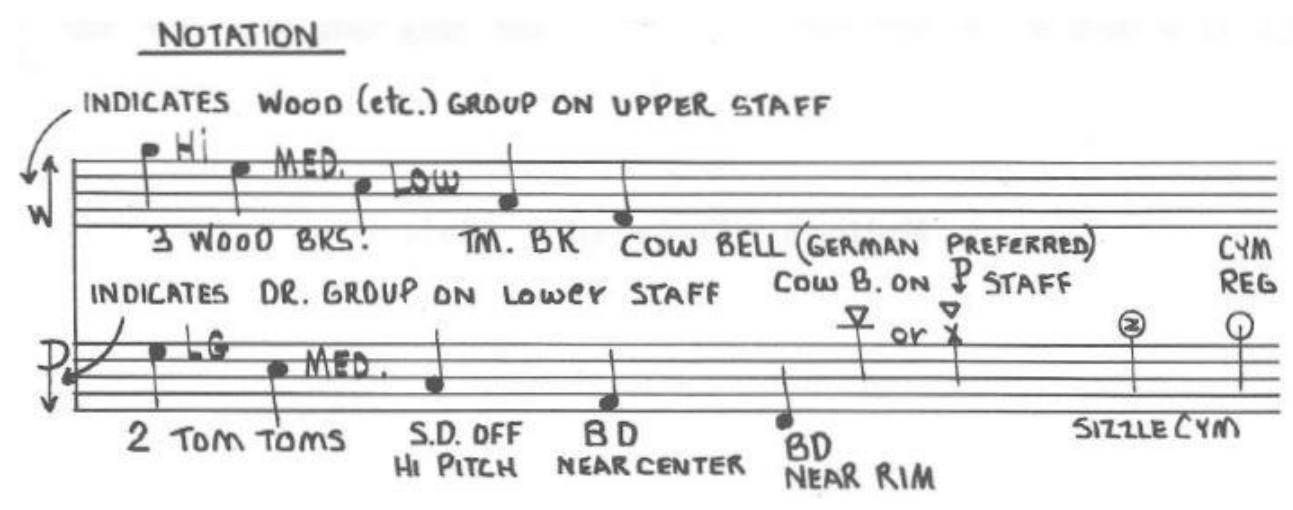

Fig. 3.3 The Love of L'Histoire, key legend

Five-line stave usage spans all levels of difficulty in the solo multiple percussion repertoire, so the percussionist can be challenged and progress in other dimensions while keeping with the notation system. Beginning students should familiarize themselves with Morris Goldenberg's Studies in Solo Percussion, a collection of twenty-six successively difficult etudes, all of which use the five-line stave. ${ }^{48}$ Seven solos in The Contemporary Percussionist utilize the five-line stave, whose heavy presence at the end of the book may suggest that technical difficulty

\footnotetext{
${ }^{48}$ Morris Goldenberg, Studies in Solo Percussion (New York City: Chappell, 1968).
} 
and notation are not innately interrelated. ${ }^{49}$ The contributions of William Kraft reinforce intermediate reading skills, developed into substantial solo pieces appropriate for a recital. The opening of French Suite contains various articulation symbols, changes of meter, and syncopation (Fig. 3.4). Using French Suite as a case study, Davis describes how educators who formulate theoretical terminology and concepts into their instruction "can enhance the learning process and ultimately lead to performances of higher quality." ${ }^{\circ 0}$ The concepts of rhythm and meter, accents concepts, metrical dissonance, phrase rhythm, and transitional techniques found in French Suite are not particularly unique to this piece and consequently should be applied to other solos within a developed percussion curriculum.

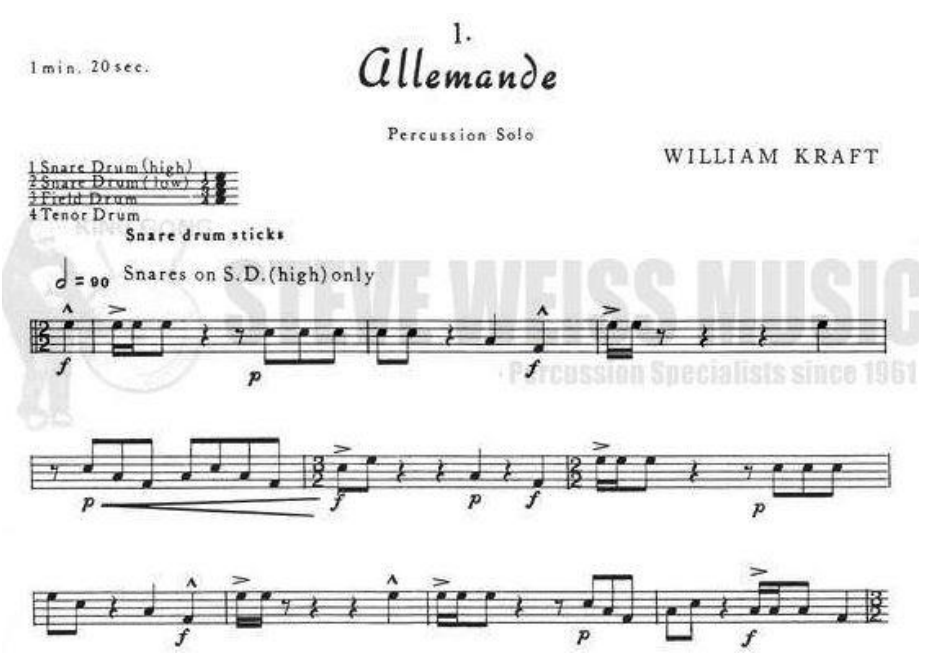

Fig. 3.4 French Suite I. Allemande, intermediate five-line stave

Iannis Xenakis Rebonds $A / B$ is an advanced solo that calls on a relatively standard notation that uses convention rhythmic notation throughout. Interestingly, Xenakis constructed a very innovative graphic notation ten years prior for Psappha (described below), so the composer

\footnotetext{
${ }^{49}$ Solos VIII, XII, XIV, XVII, XVIII, XIX and XX from Michael Udow and Chris Watts, The Contemporary Percussionist: 20 Multiple Percussion Recital Solos (Fort Lauderdale, FL: Meredith Music Publications, 1986), 20 - 21, 27, 30, 35 - 40.

${ }^{50}$ Christopher Davis, "Theories of Rhythm and Meter and Their Pedagogical Implications for Non-Pitched Percussion Music with an Analysis of William Kraft's French Suite," (DMA document: University of South Carolina, 2011), 49.
} 
was clearly aware and even fluent in applying innovative concepts to this dimension of multiple percussion. The melodic structure of the work may have been a potential influence for the conservative treatment of the Rebonds notation, especially considering its stark contrast to the polyphony and brashness of Psappha. Rebonds A is scored for nine membranophones, each occupying a space on the five-line stave (Fig. 3.5). One ledger line below the stave is used, with the lowest bass drum inhabiting the space below. Since Xenakis treats the homogenous instrumentation in a melodic way, the five-line stave intuitively imitates conventions of pitch such as contour, phrasing, and cadence.

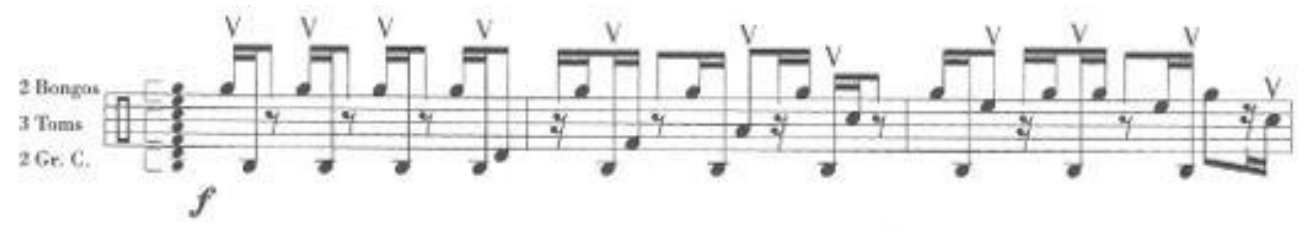

Fig. 3.5 Rebonds A, notation and key

Expanded staff notation "employs two or more staves that are bracketed together, [enabling] the composer to write for more instruments than the single conventional staff allows." "51 A parallel can be drawn between Rebonds B and The Love of L'Histoire, as each incorporates two five-line staves that are delineated by material group. Rebonds $B$ utilizes ten instruments across the two staves, bracketed together as a grand staff only at the onset of the piece and when instruments from both groups sound (Fig. 3.6). The separation of the instruments by material and the instrumentation of five instruments in each group make the score relatively easy to decipher and to read in real time. Xenakis provides further clarity by using spaces for the drums in the bottom staff and lines for the woodblocks on the top staff.

\footnotetext{
${ }^{51}$ Rachel Julian-Jones, "Notational Nomenclature for Multiple Percussion," Percussive Notes 46, no. 2 (April 2008): 18.
} 
The five-line stave and expanded staff are appropriate systems that portray the melodic structures of both Rebonds movements, which serves to reinforce the melodic perspective for the learner. Furthermore, Xenakis also uses different stem directions in Rebonds B to help differentiate notes that serve different musical functions as they occur. For example, stems up helps the performer conceptualize the rhythmic drone function of the high bongo drum, a separate voice from the ostinato in the lower-pitched drums, notated with stems down. Lastly, the similarities in notational style between both movements should be welcomed by the percussionist seeking to learn the entire piece. Ultimately, Rebonds $A / B$ presents an accessible notational task with a short learning curve, though its chief obstacles derive within the technical dimension.

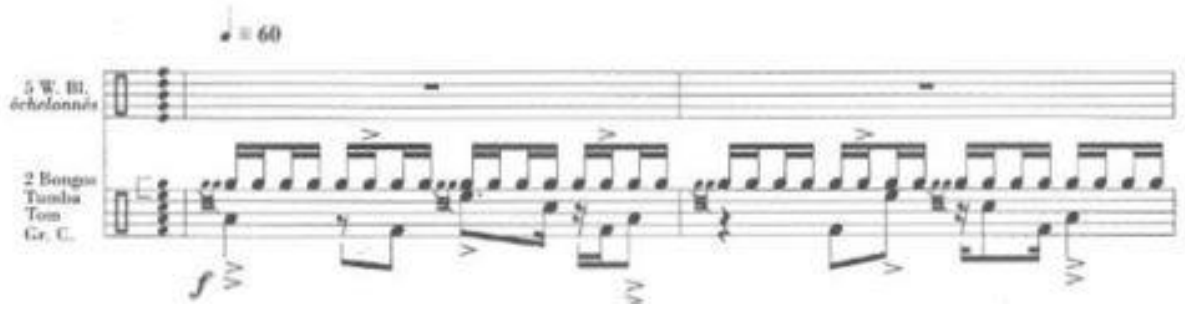

Fig 3.6 Rebonds B, notation and key

\section{Timbre Staff Notation}

Timbre staff notation applies pitch conventions of the five-line stave to multiple percussion. The timbre staff assigns each instrument to a pitch of the chromatic scale, thereby utilizing accidentals to indicate up to twenty instruments on a five-line stave. In this way, the timbre-staff applies Western melodic structures to the percussion staff, a primarily timbre-based medium. The primary benefit to the timbre staff is its incorporation of previously learned Western melodic skills, with the assumption that certain skills and concepts may transfer and thereby present shorter learning curves. 
Michael Udow was the prime figure in the development of the timbre staff system, which he incorporated in the last three solos in The Contemporary Percussionist, perhaps suggesting an advanced difficulty in its execution. ${ }^{52}$ For Solo $X X$, Udow and Watts suggest brake drums for the accidentals while various drums occupy all the naturals except a lone opera gong for \#12, the rightmost instrument (Fig. 3.7). It is unclear if the pitches of the brake drums should actually be between the two surrounding drums. In fact, the wide variance in timbre between the instruments in the solo would suggest that pitch is not the foremost factor for the composers.

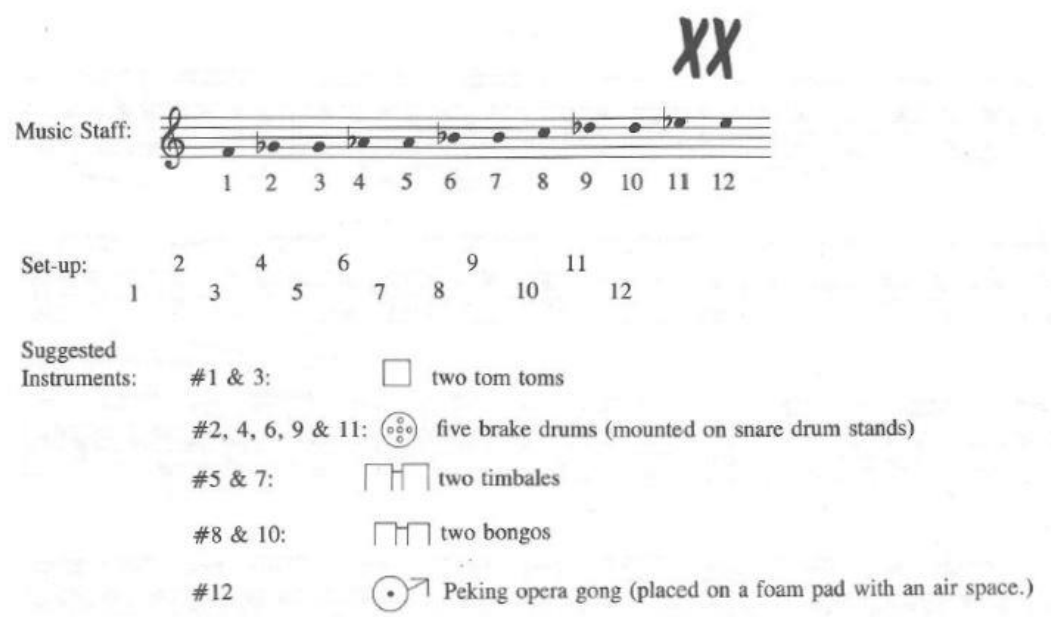

Fig. 3.7 XX, timbre staff key legend

As illustrated in the setup provided by Udow and Watts (Fig. 3.7), there is an inherent relationship between the timbre staff notation and the corresponding instrument setup; readers are meant to mimic the keyboard layout by positioning the instruments on two manuals. Percussionists simply recognize accidentals as those among the second manual, facilitating easier reading and potentially transferring skills and concepts with other musical studies. Another benefit to readability comes from the direct visual correspondence between instruments' placement and their position on the staff; instruments move to the left as staff position descends,

\footnotetext{
${ }^{52}$ Solos XVIII, XIX, and XX from Udow and Watts, The Contemporary Percussionist.
} 
and instruments move to the right as staff position ascends. An additional justification posited by Udow is the potential for the notation system to be applied to a wide repertoire of various instrumentations; percussionists experienced in the timbre staff may have progressively shorter learning curves when preparing future compositions. Instructors and students may choose to rewrite other works for the timbre staff to gain first-hand experience with its advantages and disadvantages. Udow argues that rewriting to timbre staff notation is a worthwhile and practical endeavor for select pieces such as Charles Wuorinen's Janissary Music (1966) and Herbert Brün's Stalks and Trees and Drops and Clouds (1975). ${ }^{53}$ Julian-Jones finds that timbre staff notation "can be conceived of as a truly universal approach to notating for multiple percussion." 54

However, the timbre staff system does have significant limitations in terms of how it relates to the equipment and setup dimension. The types of instrument shapes and sizes that can be used to create an effective, manageable instrument design are limited. Suspending, hanging instruments are particularly troublesome to manage and are difficult for the performer to perceive as occupying the same plane as resting instruments. The timbre staff presupposes two horizontal planes of instruments, the second of which (accidentals) alternates between groups of two and three instruments, yet many setup designs would utilize more planes and different groupings for maximum efficiency.

Although compositions utilizing the timbre staff are few, I do suggest its didactic instruction be integrated into the curriculum to create experiential learning for the student. It should be articulated to the student that such an endeavor serves primarily for historical

\footnotetext{
${ }^{53}$ Michael Udow, "Visual Correspondence Between Notation Systems and Instrument Configurations," Percussive Notes 18, no. 2 (Winter 1981): 21 - 26.

${ }^{54}$ Julian-Jones, "Notational Nomenclature," 24.
} 
understanding and appreciation, as well as an exercise in problem-solving and critical thinking, rather than a notational skill with wide application. Composer Dave Hollinden has incorporated timbre staff notation into several solo pieces that are accessible and appropriate for collegiate recitals.

A prime pedagogical contribution from the pieces of Hollinden are their position to develop skills and concepts within all four dimensions of multiple percussion. The most commonly programed is Cold Pressed (1990/94), which utilizes the timbre staff throughout (Fig. 3.8). Hollinden depicts the timbre staff clef as "a treble clef with an ' $X$ ' through it," which is the same symbol he used previously for Player 3's small percussion setup in the percussion quartet The Whole Toy Laid Down (1988). Both Udow and Hollinden find advantages in the timbre staff from a composer's standpoint, primarily in its close communication with the performer in terms of instrumentation and setup. Furthermore, aspects of musicality can also be communicated, apparent in Cold Pressed as phrase markings are present throughout. ${ }^{55}$

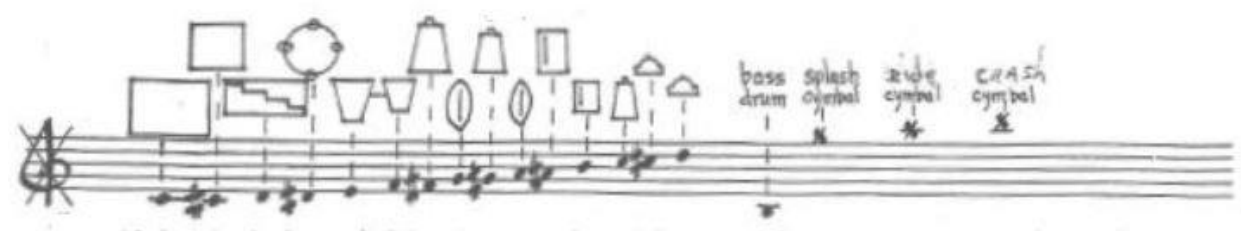

Fig. 3.8 Cold Pressed, notational key

The timbre staff contains advantages for composers and performers in all phases, from learning to finalization. From the instructor's perspective, the timbre staff serves as an appropriate learning tool that simply expands on familiar notational devices. The timbre staff can be an appropriate stage in one's curriculum that incorporates larger and more varied instrumentations and explicitly instructs the student on musical phrasing.

\footnotetext{
${ }^{55}$ Udow, 28: Julian-Jones, “A Survey of Multiple Percussion Notation,” 37.
} 


\section{Line Staff Notation}

Upon fluency of the five-line staff and experience reading the timbre staff, students should seek to gain familiarity with line staff (line score) notation. Line staff typically employs a single line to position instruments, where up to three instruments can occupy (a) below, (b) on, and (c) above the line. The application of a single line staff for the first seven solos in The Contemporary Percussionist may suggest it is more intuitive and readable than five-line. The ease of readability is especially prevalent for smaller instrumentations; the first three solos utilize two instruments, followed by three solos that utilize three instruments. ${ }^{56}$ It is not until Solo VII that a second line appears, consequently accommodating a fourth instrument (Fig. 3.9). Line scores ultimately account for fourteen of the twenty solos in the collection.

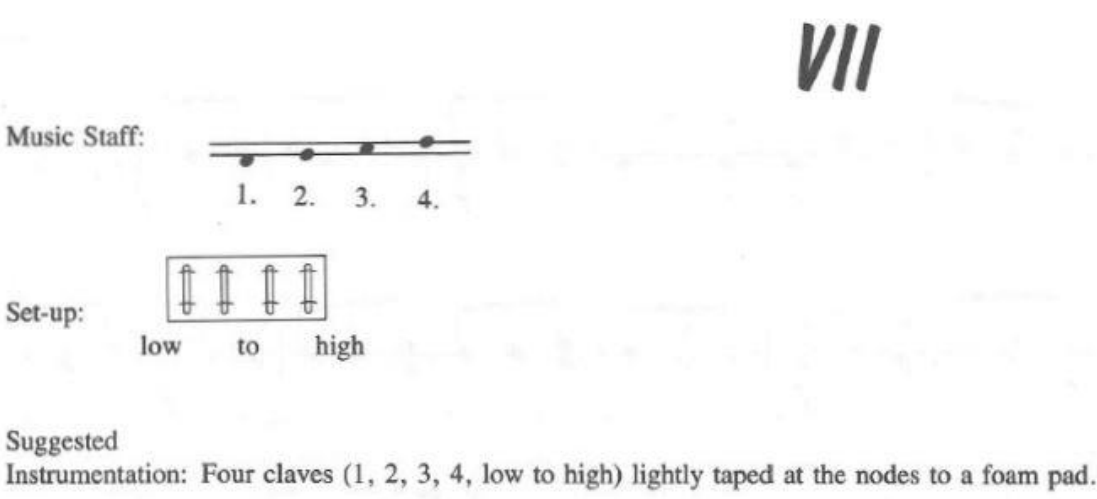

Fig. 3.9 VII, line staff with two lines

Line scores, multiple line staves grouped together by similar instruments, may pose particular challenges for the percussionist. The groups of instruments, typically classified by material, are differentiated from each other on the score by (a) larger spaces between the line groups and/or (2) each group being individually bracketed. For the short interlude that introduces “Song V" in Songs I - IX (1980 - 82), Stuart Saunders Smith utilizes a line score to visually

\footnotetext{
${ }^{56}$ Solos I, II, III and Solos IV, V, VI from Udow and Watts, The Contemporary Percussionist, 6 - 17.
} 
differentiate the maraca and table (instruments) from the voice (Fig. 3.10). The notation of multiple voices sounding simultaneously in this style might present a "slight disadvantage... [due to] the need for rests on each instrumental line or lines to account for all metrical beats." ${ }^{" 57}$ While Smith proves that this can be easily solved with proper vertical alignment and spacing, not all scores are printed as such, which can present reading challenges for performers.

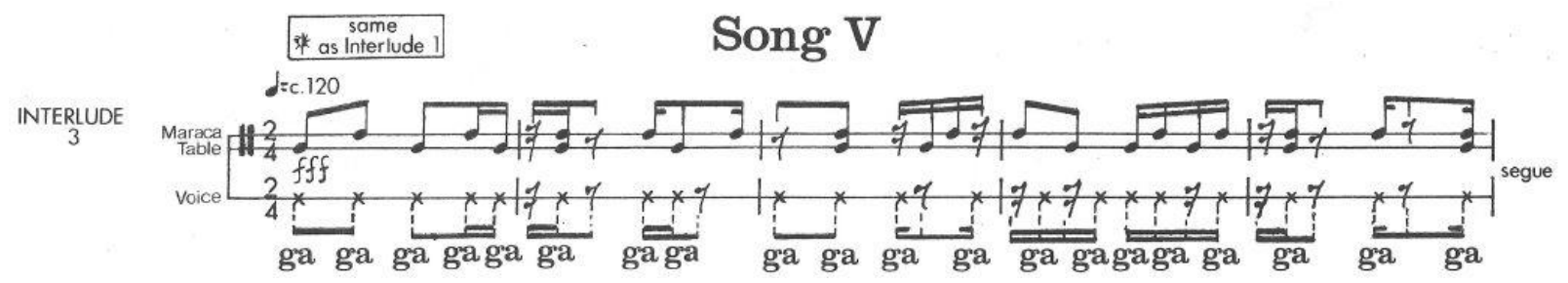

Fig. 3.10 Songs I - IX, “Song V" excerpt, line score

The line staff may provide an easier reading experience for the percussionist, but like the five-line staff, may also influence the percussionist's comprehension of the structures of the piece. Smith's notation that groups the instruments and the voice with a space between them and their separate beaming suggests that the percussionist should conceive and ultimately portray the two groups as having independent musical lines. In general, melodic and rhythmic material can be easier identified by the percussionist when notes are beamed together, which William Ortiz does for unison figures in his two-line staff system in Tamboleo (1986) ${ }^{58}$ Intuition would interpret the separation of the two pairs of instruments as two independent musical lines and perhaps each limb being responsible for one of the lines in the score (Fig. 3.11). Furthermore, the quick juxtapositions of reading rests for separate rhythms and beamed unison notes presents additional challenges for the percussionist's reading and realization of the work.

${ }^{57}$ Cook, Teaching Percussion, 81.

${ }^{58}$ Julian-Jones, "Notation Nomenclature," 20. 


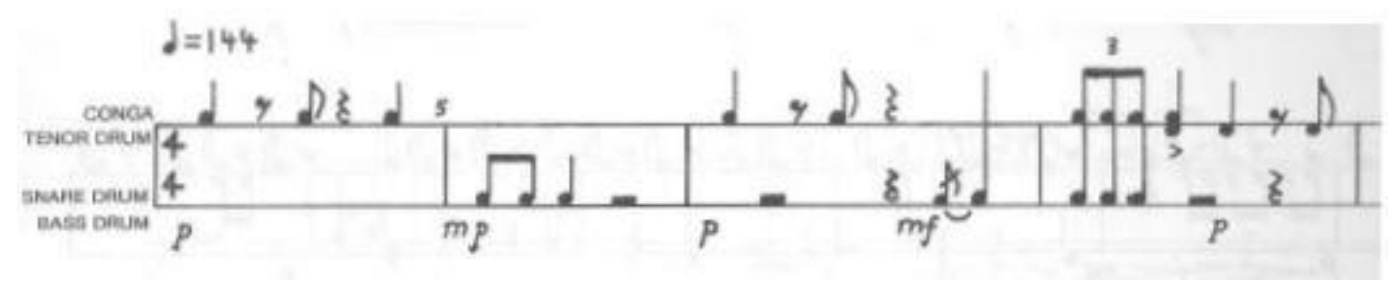

Fig. 3.11 Tamboleo, simple line score

For literature with larger instrumentation, Julian-Jones notes that line score "takes up more space on the page than other forms of notation...[, which] means more pages overall.." This is particularly apparent if each line is present even when an instrument does not sound on a stave, which results in disjunctive space between the top and bottom of the staff. Difficult reading problems and the music being printed on numerous pages can result. Ishii's Thirteen Drums is exemplary of the advanced challenges with reading line score notation for larger instrumentations (Fig. 3.12). The piece contains equally spaced lines for the twelve drums played by the hands, sticks, and mallets. A larger space is used to differentiate Drum \#13, a bass drum operated via a foot pedal.

The staves within Thirteen Drums are fully intact throughout the piece, a challenge for reading during the learning phase. Despite the challenge, this is an appropriate approach because the homogenous instrumentation emphasizes their pitch relationships and resulting melodic structures. Visually corresponding the vertical placement of the drums within the 12-line bracket with precision is quite difficult, especially in the middle and right of the stave, farthest from the drum indicators provided on the left. During my learning phase of the piece, I made a mark that indicated the drum right above each note.

Upon learning the piece, I sought to shift my perspective from simply relying on measuring the distance from the top or bottom of the staff or shifting my sight to the left to locate 
the indicators for each drum, to perceiving the "intervals" between the drums within the musical material itself. Despite the long learning curve, understanding the intervallic relationship between the instruments ultimately made reading easier and even illuminated intervallic and harmonic structures, in the music that I might have missed otherwise, arguably allowing myself to play more musically

The reading of rhythmic patterns, note durations and rests also present a challenge. Ishii chooses to beam for much of the piece, which is helpful in deciphering the rhythmic composite between the drums. However, there are instances in Thirteen Drum where unbeamed rhythms and staff-wide sixteenth rests pose reading problems (Fig. 3.12). The omission of a time signature, predominance of grace notes and rolls in this excerpt presents advanced problems common throughout much of the solo repertoire, suggesting that the structure of meter be abandoned for more rhythmic, gestural and interpretive freedom.

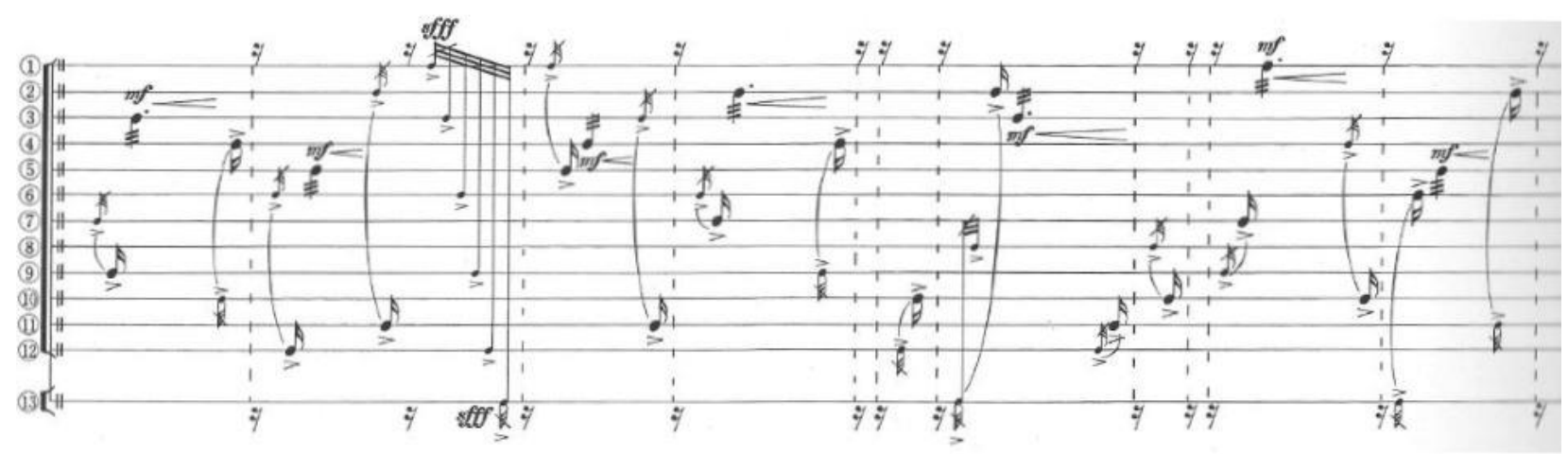

Fig. 3.12 Thirteen Drums, unbeamed excerpt

In Parson's Piece (1968), William Hibbard imparts an innovative solution to meter issues in line score. Hibbard notates the "attack rhythm" on the top of each stave so that the rhythmic composite is readily available without the need for the percussionist to measure notes and rests on multiple lines (Fig. 3.13). The rhythmic composite isolated on the top of the staff also encourages exact placement of simultaneously sounding strikes, regardless of orchestration and 
dynamic balance provided on the bottom four lines. The separation and individual manipulation of rhythm, orchestration, and dynamics is a necessary technical skill that can be developed using the unique notation in Hibbard's piece.

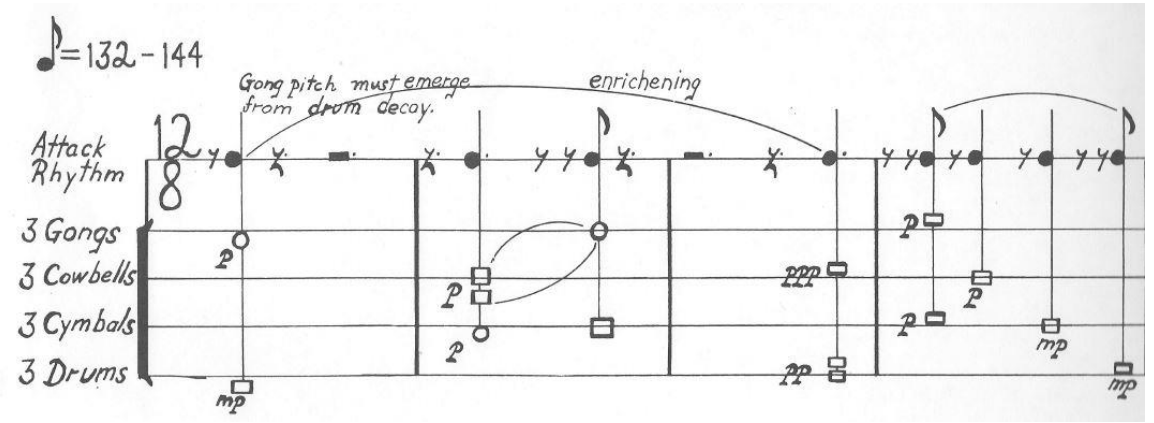

Fig. 3.13 Parson's Piece, line score with rhythmic composite

\section{Real-time Symbols}

The percussion student must be prepared to read a variety of symbols that communicate real-time information. Of course, percussionists should be fluent in reading expression symbols common to all modern Western scores, such as changes of tempo, dynamics, and phrase markings. Further real-time symbols are rather particular to multiple percussion notation, such as explicit instructions to change implements. These instructions manifest via text descriptions, abbreviations or shorthand, and pictogram symbols.

In Latin Journey (1998), Mancini provides short instructions of the necessary implement changes specific to each hand, as well as other performance techniques such as turning snares on or off (Fig. 3.14).

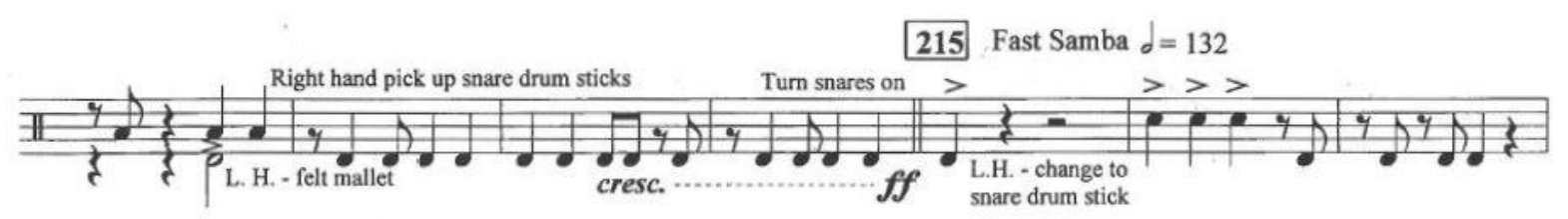

Fig. 3.14 Latin Journey, real-time descriptive instructions

In Inspirations Diabolique, Tagawa poses a different solution to a similar problem by integrating unique symbols in the score with footnotes (i.e., "1," "2," etc.) that direct the reader 
to the bottom of the page for performance directions (Fig. 3.15). Integrating these directions takes a degree of score study, as well as either memorization or integration of their own shorthand that can be read during performance.

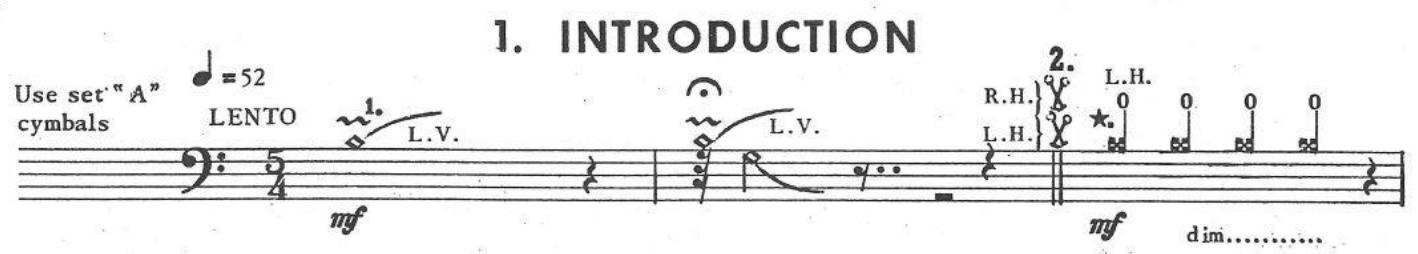

Fig. 3.15 Inspirations Diabolique, extended technique, implement, and note-head symbols

The percussionist also must be aware of various note-heads, which composers often use to indicate a different playing spot or even multiple instruments that occupy the same position on the staff. In The Love of L'Histoire, the space above the drum group staff is used to indicate the cowbell, sizzle cymbal, and regular cymbal, albeit with differing note-heads (Fig. 3.3). The percussionist must pay particular attention to the cowbell in the score, as the one instrument has two positions on the drum group staff as well as one position on the wood group staff, each notated with a different note-head. Inspirations Diabolique contains the common technique of using pictograms to indicate implement changes; the symbols above the double bar line indicate that four "wound musser mallets" are to be held (Fig. 3.15). Larger and varied instrumentations typically utilize written abbreviations or pictogram symbols that indicate a change of instrument or instrument group on the score as it occurs in real time. A percussionist's ability to navigate these symbols is imperative due to their prevalence in chamber and large ensemble works.

However, there are performance challenges that emerge from text abbreviation and pictogram symbols. Julian-Jones notes challenges in deciphering text abbreviations due to potential language barriers between the composer and performer, but she also recognizes that 
pictograms are not universal, either. ${ }^{60}$ Percussionists might benefit from the research of Gardner Read, who collected and analyzed hundreds of scores and performance notes in works published between 1950 and 1989 to create comprehensive lists of various pictogram symbols. ${ }^{61}$ One of the many difficult aspects of the notational dimension in Stockhausen's Zyklus No. 9 (1959), a graphic and spatial score, lies in recalling the meaning of the pictograms as they occur (Fig. 3.16). Since the music can be flipped in either direction, the pictograms are placed on both sides of the musical information that appear on staves of varying construction. The large, abrasive, and likely unfamiliar shapes that dominate the page pose significant reading problems for those that confront learning the piece.

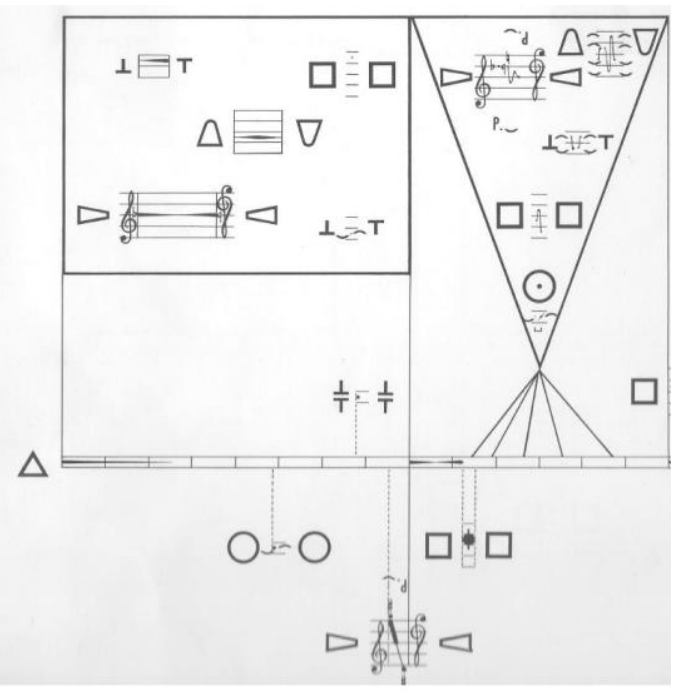

Fig. 3.16 Zyklus, pictograms on the score

Five-line, timbre staff, and line score are typically considered standard notations if they use conventional Western rhythmic values. These notational devices may serve well as a foundation for students as they progress through the music program. Interestingly, percussionistscholars have debated the appropriateness of rewriting works into another standard notation, one

\footnotetext{
${ }^{60}$ Rachel Julian-Jones, “A Survey of Multiple Percussion Notation with an Emphasis on Timbre Staff Notation and Setup” (DMA document, University of Nevada, Las Vegas, 2005), 9 - 10.

${ }^{61}$ Gardner Read, Pictographic Score Notation: A Compendium (Westport, CT: Greenwood Press, 1998).
} 
that is more effective, efficient, or simply preferred by the individual. Cook offers the same etude written in six different notation styles "to give the percussion student the knowledge and option to re-notate certain passages, to make performance more expedient, and/or to improve composition." ${ }^{62}$ By "promoting the appealing aspects of timbre staff notation and setup," we would infer that Julian-Jones - as well as Udow, for that matter - would suggest re-notating music onto the timbre staff whenever possible. ${ }^{63}$ On the other hand, Petrella discourages the practice of re-notating music, choosing to trust the composer's intentions and directions. ${ }^{64}$ Despite their opposing perspectives, each of these scholars recognize the pedagogical view that encourages students to gain experience in all types of notation, and certainly discourage the reliance on just one. They appear to be in agreeance that rewriting scores should not be applied to unconventional notational systems such as graphic, spatial, and text.

\section{Graphic Notation}

Graphic notational devices apply a system of abstract symbols that stress implicit directions that are typically specific to each piece. Graphic scores may resemble visual art rather than a musical notation per se, a movement Julian-Jones claims commenced with Earle Brown's Folio (1952 - 53). ${ }^{65}$ To this extent, composer Herbert Brün claims that these "graphic displays turn into scores as soon as the interpreter translates their structural characteristics into the instructional code of another medium.. ${ }^{.66}$ Despite their artistic merit and lack of explicit directions, improvisation is typically an "irresponsible approach, at best." ${ }^{67}$ The subjectivity of the notation inherently encourages individuality by relinquishing select compositional freedoms

\footnotetext{
${ }^{62}$ Cook, Teaching Percussion, 80.

${ }^{63}$ Julian-Jones, “A Survey of Multiple Percussion Notation," 61.

${ }^{64}$ Petrella, The Multiple-Percussion Book, 10.

65 Julian-Jones, “A Survey of Multiple Percussion Notation,” 23.

66 Julian-Jones, "Notation Nomenclature," 22.

${ }^{67}$ Applebaum, "Multi-Percussion Performance Problems," 70.
} 
to the performer, but Brün emphatically stipulates that the interpreter is "asked not to improvise. He is asked to compose." 68 To successfully develop the skills of being a part-composer, such as negotiating indeterminacy and improvisation through visual representation, they should be nurtured within the percussion curriculum.

In graphic scores, musical information is indicated by various shapes, lines, dots, numbers, and countless other artistic creations that have varying levels of instructions attached to them. The Multiple-Percussion Book introduces readers to graphic means by blending unconventional lines of various weight and angles with conventional symbols such as breath marks, fermata, and quarter rests. The Graphic by Design etude uses precise staves that are read in the traditional left-to-right and top-to-bottom manner (Fig. 3.17), which is a welcomed introduction to graphic notational devices since the serious repertoire represents extreme undertakings. Interestingly, Udow and Watts do not offer a solo with graphic means in The Contemporary Percussionist, choosing to notate sounds with conventional note heads throughout the collection.

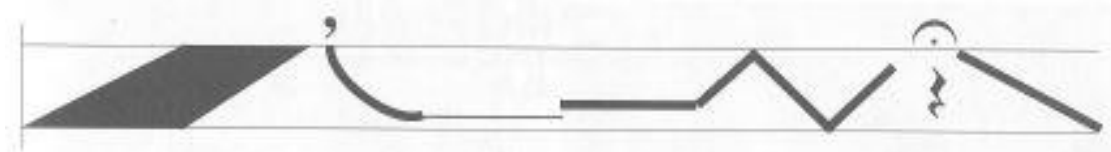

Fig. 3.17 Graphic by Design hybrid symbols

While it is simply infeasible for any written notation to capture the complexity and detail of a music performance, graphic notations impose additional problems that are not present in standard notational symbols. The question of transferability is a legitimate one, the fact that standard notational conventions generally transfer among pieces in the Western art form is less overt among pieces in graphic notation. Although generalizations are illusive due to their

\footnotetext{
${ }^{68}$ Julian-Jones, “Notation Nomenclature," 20.
} 
individuality, a degree of indeterminacy and the performer's responsibility to act as partcomposer is inherent with any realization. According to Applebaum:

It is essential that a performer playing a work that incorporates notational practices understand how much freedom that system allows. Without an awareness of the mechanical and interpretative aspects of the notation system, a performer will probably distort the composer's intent... ${ }^{69}$

Score study in the realm of interpreting the composer's intentions and how facets of indeterminacy are controlled may be relatively foreign concepts to percussionists who only have experience in reading standard notation. It should be noted that a degree of interpretation and translation is necessary in all styles of written music, from interpreting and improvising Baroque ornamentations to deciding on the number of repeats for patterns and cells among the $20^{\text {th }}$ century minimalist style presented by composers like Terry Riley, Frederic Rzewski and Steve Reich. Therefore, the development of sympathetic, empathetic translation skills should be a student outcome of any collegiate percussion curriculum.

Precise rhythms are rarely specified, and instead performers are guided on to other musical elements such as instrumentation, implement, dynamics, density, or texture. In the King of Denmark (1964), Morton Feldman constructed a grid that poses reading problems that the percussionist must seek to solve immediately. The relative register of the instruments is notated on the corresponding low, middle, and high rows of the staff (Fig. 3.18). Only in select moments does the composer explicitly specify which instrument material is to be used for a specific passage. The tempo of the piece is also semi-structured, each box representing between 66 and 92 beats per minute. Feldman retains control of the material's rhythmic density by specifying numbers that indicate the number of sounds to be played in each box. Roman numerals are used to depict simultaneous sounds, while integers are used to indicate "single" sounds played in a

\footnotetext{
${ }^{69}$ Applebaum, "Multi-Percussion Performance Problems," 70.
} 
rhythm. Other symbols are used to depict a quality, duration, or number of sounds, but the performer is consistently responsible for composing the rhythm and exact placement of the strikes within the box. The close relationship between the notation and the indeterminate instrumentation, as well as the resultant problems with setup, are compounding performance problems that make The King of Denmark a monumental, advanced work for solo percussion.

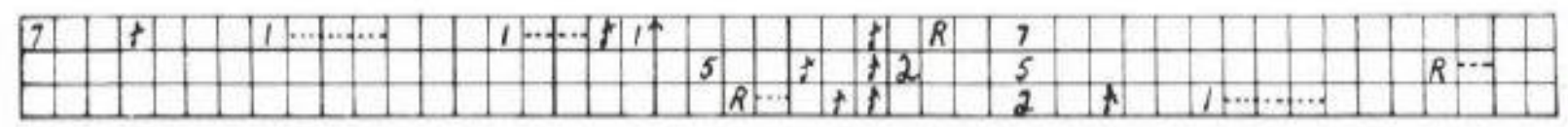

Fig. 3.18 King of Denmark graphic score

The importance of the performance notes is the primary avenue for (a) composers to provide descriptive instructions and (b) for the performer to receive tangible information. Foregoing analysis of the performance notes would have dire consequences when preparing a graphic work. In addition to the traditional instructions such as setup design and instrumentation, Stockhausen provides detailed instructions on the eight notational structures that occupy the pages of Zyklus (Fig. 3.16). Without comprehensive understanding of the further performance suggestions the composer provides, a realization of the piece is arguably meaningless if not utterly unattainable. Since this information is typically provided in prose form in the composer's first language, the attention to detail in a student's translation of these instructions into English is paramount. More detail regarding the graphic score for Xenakis' Psappha is provided at the end of this chapter.

\section{Spatial Notation}

Spatial notational techniques use the physical space on the page to communicate aural variables to the performer. Julian-Jones notes that spatial notation is "often combined with 
graphic characteristics" so that the differentiation between the two is easily blurred. ${ }^{70}$ Spatial aspects limit the precision of a certain value used to measure a specific parameter. An optimal introduction to spatial notation would limit just one parameter, such as Solo XVIII from The Contemporary Percussionist, which implicitly instructs rhythm (Fig. 3.19). To limit temporal parameters, the composers omit the time signature and bar lines. Note attacks, durations, and composite rhythm are merely implied by the placement of dots and the relative proximity to one another. In addition to the dynamics provided below the staff, the composers maintain meticulous control over the pitches and the order the pitches occur in a performance, while the performer has the responsibility of determining their exact rhythmic relationship, i.e., the space. Interestingly, this is the only solo in the collection in which Udow and Watts utilize spatial notation. $^{71}$

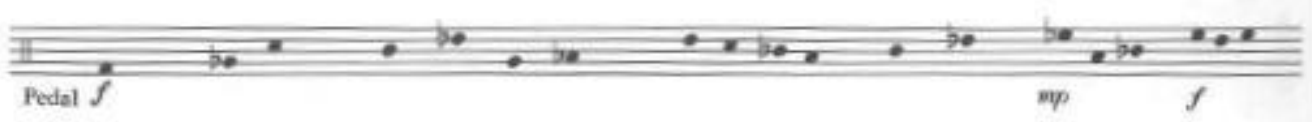

Fig. 3.19 XVIII, spatial treatment of rhythm

For Spatial Choices, Allemeier provides the performer with instructions for instrumentation: a minimum of three woods, three metals, and three membranes, which are explicitly notated on the top, middle and bottom row of the score, respectively (Fig. 3.20). The performance notes further instruct that while "relative pitch is indicated by the vertical placement of a note head in an instrumental line...dynamics are indicated by the size of the note head, e.g., a larger note head corresponds with a louder dynamic." ${ }^{72}$ Despite this nontraditional notational device, the score does progress in the traditional manner, left-to-right and top-to-bottom, in

\footnotetext{
70 Julian-Jones, "Notation Nomenclature," 23.

${ }^{71}$ Solo $X V$ also omits bar lines and conventional rhythmic values. However, since each dot is instructed to be 208 $\mathrm{bpm}$ and there are no other durations (or rests), the performer is explicitly instructed on the rhythm in the work.

72 Petrella, 73.
} 
which each stave lasts between 40 - 60, assumingly, beats per minute (bpm). The performer must compose precise rhythms based on the approximate placement in relation to each other and to the grid. In this way, the composer explicitly describes the instrumentation, pitch, dynamics, and rhythmic parameters, albeit those directions must be interpreted by the performer via implicit spatial locations in the score.

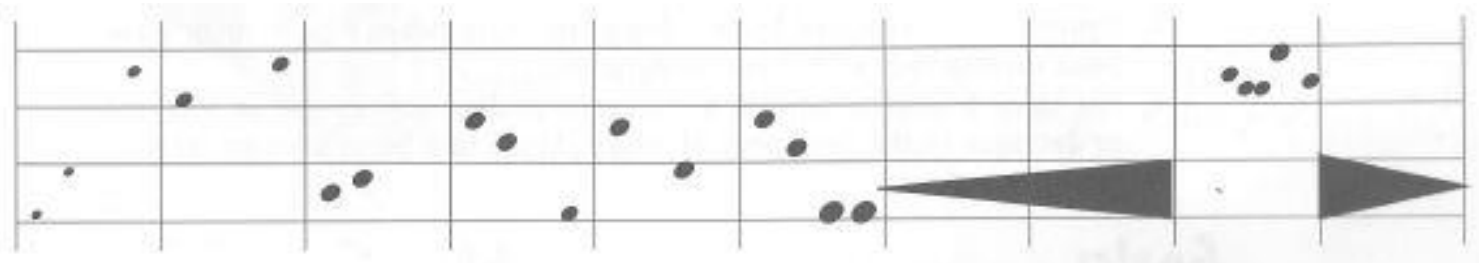

Fig. 3.20 Spatial Choices, spatial treatment of pitch, dynamic, and rhythm

The piece 27'10.554' by John Cage is exemplary of spatial notation within full-length, serious works (Fig. 3.21). Cage provides one page of performance notes to accompany the score, in which he notes that "a correspondence between time and space is made so that each page $=$ one minute."73 Symbols for the sound occurrences are provided on the score, yet, much like the previous graphic and spatial examples, their exact placements require interpretation. Integers above the staff aid performers by indicating the total seconds into the performance, but since vertical lines are not provided, the responsibility of its rhythmic realization is ultimately left to them.

The percussionist who seeks to perform 27'10.554" must navigate the explicit and implicit instructions provided by Cage. Facets of the instrumentation are implied by the different symbols that occur in the score, one such being longer durations represented by lines that move left to right. Its distinct symbol might suggest that single dots are sounds of extremely short duration, which would be aesthetically appropriate given the dense texture where these symbols

\footnotetext{
${ }^{73}$ John Cage, 27'10.554" for a Percussionist (New York, C. F. Peters Corp., 1960), notes.
} 
are dominant. A survey of the entire score is needed to illuminate that an instrument that can produce longer durations is required within each group. The metal group (M) contains the longest duration, a 12-second sound in which both crescendos and diminuendos occur. This is likely to be orchestrated most easily on a vibraphone, which maximizes usage as it can also produce short, articulate sounds.

Counter to the convention of relating the vertical $y$ axis to pitch, Cage applies the relative dynamic to the vertical orientation. Each group's single line in the staff represents a mezzo-forte dynamic. As lines indicate sounds with extended durations, an angled line instructs a change in dynamic throughout a sound's sustain. For sounds that are notated between two different instrument group lines, Cage uses a stem attached to the dot to help determine its position. Despite the stems, there are still times when the performer must decide whether such a sound is depicted as (1) an extremely loud sound of the group notated below or (2) an extremely quiet sound from the group notated above.

Cage combines explicit and implicit instructions for the performer to realize $27^{\prime} 10.554$. The composer supervises the performer in instrumentation, temporal and dynamic decisions, but the performer still has much responsibility in those parameters. Cage disregards informing the pitch component in both performance notes and score notation, but the performer should still consider pitch, rather than ignoring it completely. The listener would instinctively perceive the pitch component, so a performer should integrate pitch relationships into their realization as well. Many performers take up Cage's suggestion for "the aid of a recording," alleviating some of the restraints presented by note density, instrumentation, and extended note symbol restraints. ${ }^{74}$ Percussionists may find that using pre-recorded and/or electronic audio to accompany their live

\footnotetext{
${ }^{74}$ Ibid.
} 
performance will relieve equipment setup and technical demands, but the notational undertaking of interpreting this spatial and graphic score remains at a very high level.

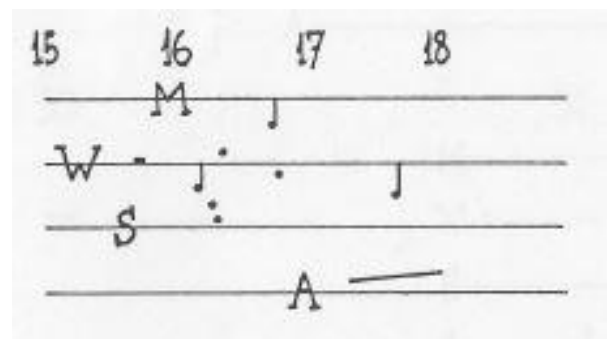

Fig. $3.2127^{\prime} 10.554^{\prime \prime}$, spatial notational devices

Herbert Brün presents a graphic and spatial score demanding the highest caliber of interpretive skills in Touch and Go (1967). Standard notational conventions are all but abandoned for this work, with Brün instead choosing a variety of different graphic symbols to communicate very specific information. A quadrangle is used to represent the sonic information, while thirteen different note-head symbols are used to represent the different implements or part of the hand. Spatial devices are also innovatively used and applied to each of the symbols. Firstly, a quadrangle's placement along the y-axis (“distance") indicates such factors as pitch and sharpness, an interesting marriage of sound properties. The placement of the note-head (implement) along the "distance" axis indicates the sound's dynamic. Furthermore, the angle of the stem on the implement note-head indicates the "action of arm and hand" used for the performer's attack (i.e. fingers, wrist, arm or combinations), while the size of the implement symbol indicates the "flight-path" and resulting resonance of the sound (straight and dead vs. circular and resounding). Further performance notes describe the treatment of silence durations when moving from page to page in the score to create phrases; sentences whose time between statistically decrease through a performance. These details suggest that a realization of Touch and Go requires expert navigation of both explicit and implicit instructions; while Brün provides 
a complex notational system that governs a copious amount of details, the performer claims many compositional and interpretive freedoms.

\section{Text Notation}

Written prose descriptions and incorporations of text serving as musical instructions provide a unique set of performance problems for the percussionist. Text notations account for a vein of musical compositions that pull influence from the American performance artist groups surrounding John Cage in the second quarter of the twentieth century. Written prose scores may resemble the "performance directions" section of a conventional multiple percussion piece, in which there is no other accompanying score. Of course, the percussionist must read through the entirety of the score before beginning its preparation, stressing creative problem-solving at an early stage of learning a piece. The act of performance may also present a different style entirely, as performance of these works are far removed from reading a detailed instructional script during performance.

Pauline Oliveros's The Single Stroke Roll Meditation (1988) is an appropriate introduction to the style, in part because the printed words are legible and the instructions both descriptive and articulate. ${ }^{75}$ The limit of the piece to a single snare drum also allows the percussionist to focus on satisfying Oliveros's instructions, fully exploring the timbral possibilities and performing with purposeful direction and virtuosity.

John Cage's Composed Improvisation for Snare Drum (1987) represents a more advanced notational task. While the score is quite legible, it does not contain quite as descriptive or articulate language as Oliveros. Compounded variables structure the work, demanding close attention to detail as well as empathy for the composer's intentions. The performer must carry

\footnotetext{
${ }^{75}$ The Single Stroke Roll Meditation from The Noble Snare vol. 4 (Sharon, VT: Smith Publications, 1990).
} 
out chance operations to determine these variables, which may occur as late as the onset of the performance. ${ }^{76}$ Publicly determining integral aspects of the performance challenges the performer's command of their improvisation vocabulary and exposes them to an audience that now has structural knowledge of the piece. Cage's Child of Tree (1975) contains a similar style, as a prose description informs the performer of the chance operations that structure the improvised performance. However, the illegibility of the handwritten style and the ambiguity of Cage's corrections, such as crossed out and inserted words, makes interpreting this score and creating an informed realization a challenging task.

Other examples of textural notational devices in the advanced percussion literature integrate text without the structural confines of prose, thereby representing a form of poetry. In Songs I - IX, Smith portrays sections in strict poetry that are void of musical instructions. Select passages in the work also integrate poetry with symbols attached that instruct musical qualities to the recitation. Elaborate performance directions are provided (a) in the "general directions" and "performance directions" that are isolated from the score, (b) attached to the written staves and other symbolic notations, and (c) aside from the musical score in the form of "directions," "further directions," and footnotes. These textual components are integral to a realization of the piece, such as the further directions for "Song VI, \#D" that differentiate the performance based on the performer's gender:

...if the performer is a man, then he performs, "Oh yeah... of you" in a falsetto, i.e. imitates a women's voice. But if the performer is a woman, she performs, "Because... of me!" in an exaggerated low masculine voice."77

\footnotetext{
76 John Cage, "Composed Improvisation for Snare Drum," in The Noble Snare, vol. 2 (Sharon, VT: Smith Publications, 1990).

${ }^{77}$ Stuart Saunders Smith, Songs I - IX (Sharon, VT: Smith Publications, 1981), music score.
} 
Other textual devices use elements of language as the primary system for indicating musical directions. Stuart Saunders Smith uses various notational styles to synthesis text and music throughout his compositional output, in which percussionists must consider diction and the musical details of spoken language. Solfin discusses the two styles of his texted works within Smith's repertoire: (a) text that suggest a narrative and (b) speech-songs, whose text retains a portion of proper syntax or semantics while abandoning others. ${ }^{78}$ Recitation of texts that abandon proper linguistic structures can be a challenge for the performer's establishment of spoken rhythm, pitch, and cadence, as well as an interference to concentration, memorization, and dramatic presence.

The works of Stuart Saunders Smith are exemplary of the poetic style of text notation, but they commonly blend conventional, graphic, and spatial devices as well. All four notational types are used in the various songs of Smith's Songs I - IX, which utilizes narrative text, speechsong, and nonsensical vocables. While attention to detail was taken on the part of the composer and Smith Publications to maximize legibility, Songs I - IX remains a notational tour-de-force. Smith's blend of poetry and text notation with various graphic symbols that informs the percussion accompaniment in Tunnels is described in detail under the lens of performance conception in Chapter 5.

Instructors would do well to establish text notations into their curriculum, but linguistic practices may be completely foreign to the percussionist. These types of notational devices are becoming increasingly common in solo and chamber ensemble literature as well. Given the increasing presence of new music ensembles, it is our job as percussion instructors to prepare the student for these experiences. Without the confines of reading precise notes that can be

\footnotetext{
${ }^{78}$ Paraphrased from Elizabeth Solfin, “Text as Music, Music as Text: Stuart Saunders Smith's Works for Percussion and Spoken Word," (PhD diss., The University of Arizona, 2017), 21 - 23.
} 
interpreted as "right or wrong," realizing graphic, spatial, and text scores may intuitively increase the percussionist's sensitivity to interpretation, dramatic acting, and delivery, which are aspects of the conceptual performance dimension that should be integrated into percussion curriculum as well (see Chapter 5).

\section{Iannis Xenakis, Psappha (1975)}

Performed on April 13, 2014, DMA Recital in Falbo Theater, West Virginia University. Iannis Xenakis composed Psappha for Sylvio Gualda in 1975. The instrumentation is indeterminate; the performance notes specifies six instrument groups split into three categories: skins, woods, and metals. Xenakis details the instrument groups, their relative register, and provides possible instruments in the key legend provided in the back of the score, albeit in French, which will require careful translation. Instrument groups are given an uppercase letter via the order of their entrance in the piece, groups A, B and C are groups of either wood or skin and groups D, E, and F are groups of metals. Except for the single E instrument, each group calls for three instruments in a group, whose stave assignment intuitively correlates with the high/middle/low of their register (i.e., relative pitch). Referenced in Chapter 2, Sallak makes detailed conclusions regarding how the piece's technical demands inherently affect instrumentation choices. $^{79}$

The notation of Psappha presents a barrage of challenges for the percussionist. Understanding the vertical $y$ axis was my first objective in learning the piece because it could be done completely detached from the constraints of time. Xenakis assigns each of the piece's sixteen instruments to its own horizontal line, which are then bracketed within each group, designated by an uppercase letter. The spaces between the horizontal lines are not used, which

\footnotetext{
${ }^{79}$ Bill Sallak, "Informed Indeterminacy."
} 
probably renders the score much larger than necessary, although not unprecedented (see Thirteen Drums, Fig. 4.16). The large staves are coupled with the large physical size of the score, which renders photocopying difficult, unfortunate because making extra copies is likely desired to make interpretive marks or to place duplicate copies of sheets at different locations in the setup. However, the large score did prove beneficial, as it was easily readable despite being positioned far from me because of an extensively large setup design.

To preserve some vertical space, Xenakis eliminates instruments and instrument groups from the score that do not sound on a staff. Hiding the empty staves changes the size of each system, requiring the percussionist to double-check the staff designations at the left alignment for every system. The instrument assignments shift in the finale of the piece, when $\mathrm{F}$ is suddenly positioned above C (Fig. 3.22).
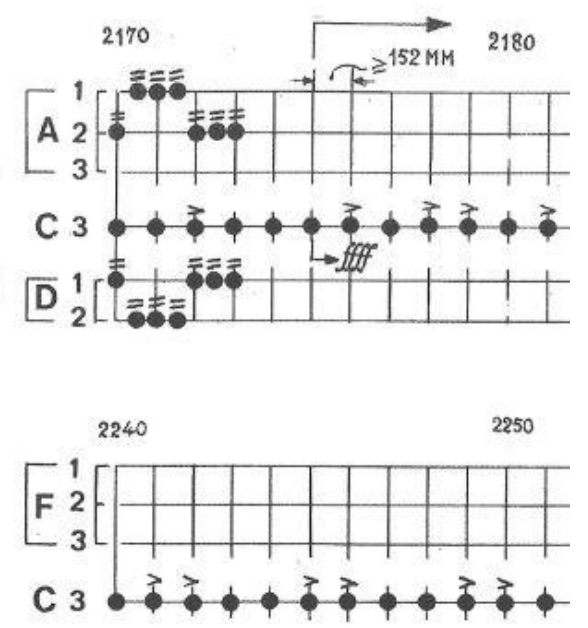

Fig. 3.22 Psappha, final staves

The piece progresses in the traditional manner (left-to-right and top-to-bottom).

Movement along the horizontal $x$ axis uncovers vertical lines that represent an equal duration of time. Each vertical line represents a unit of time that is related to a traditional metronome marking, albeit the tempos provided by Xenakis are described as minimums. A performer might 
interpret the progression of each vertical line as the fastest pulse, likely perceived as downbeats or quarter notes, in which case the tempo of the work is quite fast. Sounds are indicated by dots that appear in one of two relationships to vertical lines on the score, those on the vertical line and those directly between two vertical lines.

The uniform weight and size of the vertical lines might suggest a perception of an even, fast pulse, but the performer is free to translate the dots into any standard rhythmic value or combinations of values throughout the piece. Equating dots on the vertical line to quarter notes would certainly give the piece driving motion and energy. However, the performer is free to change their conception at will, as there are no implied meters throughout the piece. While the sound entrances are explicitly prescribed, itself a stark contrast to King of Denmark, the performer chooses how they may conceive of their groupings and subsequently whether to portray meters in performance. The choice is immediately relevant, as the performer can choose to interpret the accents at the onset of the piece (a) as downbeats in changing meters or (b) accented syncopations (Fig. 3.23). In the former interpretation, the first note of the piece would be considered a pickup, as it is an unaccented note followed by an accented note.

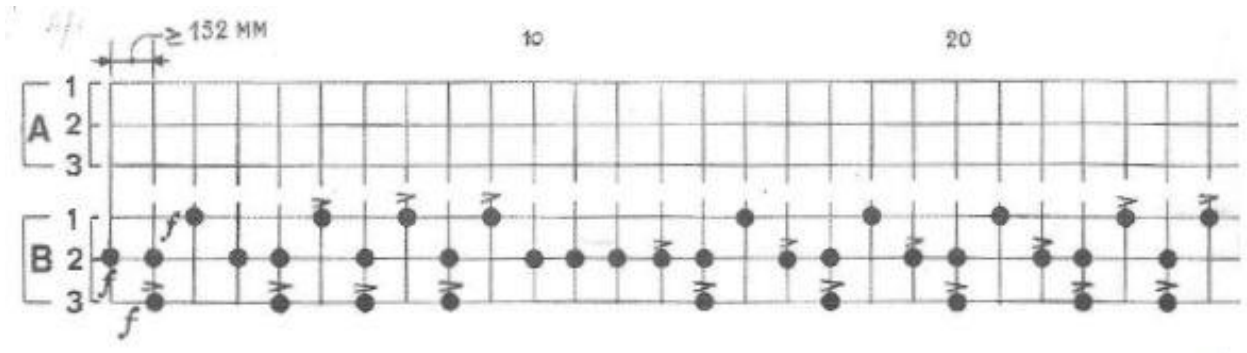

Fig. 3.23 Psappha, opening

A similar method of notation was developed by Phillip Harland and James Koetting, who sought an effective system for notating traditional African music to help their students in world 
music ensembles at UCLA. ${ }^{80}$ Koetting details the time-unit box system (TUBS) as having distinct representative advantages to traditional African music, one being its visual representation of subjective meter. In the emic perspective, traditional sub-Saharan African drum ensemble musicians consider the relationships between their patterns. An individual pattern might have a downbeat unique to the other patterns of the ensemble, a process A. M. Jones called staggeredentry. ${ }^{81}$ Similarly, Chernoff used the term subjective beat to describe the traditional Africanism that grants performers their own interpretation of meter for the different patterns of the ensemble or even the same individual pattern, for that matter. ${ }^{82}$

In addition to the freedom of perspective, TUBS notation also more effectively portrays interrelated rhythmic patterns. In Fig. 3.24, Koetting uses the 3:2 polyrhythm in TUBS to display the correlation, arguing that "Western notation, in treating the drum ensemble patterns as relatively self-contained units, fails to represent adequately their close relationship." 83

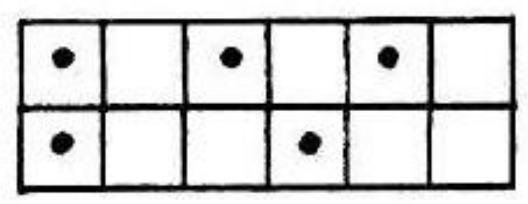

Fig. 3.24 TUBS 3:2 Polyrhythm

The grid score makes the interrelationships between the patterns easily legible; sounds that strike simultaneously simply appear as dots on the same vertical line. Painstaking calculations often arise to determine rhythmic placement conventional notation due to inaccuracies of vertical alignment and note spacing in publications. These are compounded in the

\footnotetext{
${ }^{80}$ James Koetting, “Analysis and Notation of West African Drum Ensemble Music," Selected Reports in Ethnomusicology 1, no.3, 115 - 146.

${ }^{81}$ A. M. Jones, “African Rhythm,” African 24 (1954): 41.

${ }^{82}$ Waterman quoted in John Miller Chernoff, African Rhythm and African Sensibilities (Chicago: University of Chicago Press, 1979), 50.

${ }^{83}$ Koetting, 128.
} 
latter half of measures due to rests and the need to work backwards. Since vertical alignment is intact throughout and there are no implied meters, these reading and comprehension problems characteristic to standard notation are not present in the graphic score of Psappha.

Durations of sounds are not prescribed in the score; all sounds are indicated by black dots. The performer is thereby granted the freedom to interpret the length of sounds. Equipment choices, technical tactics, and performance approaches to executing and portraying sound duration are described in their respective chapters. In the notation dimension, the percussionist must recognize if, when, how and why the composer provides implicit or explicit directions onto sound durations. Figure 3.25 shows a particularly open section of the work, whereas the performer may choose (a) a disjunct or (b) fluid interpretation.

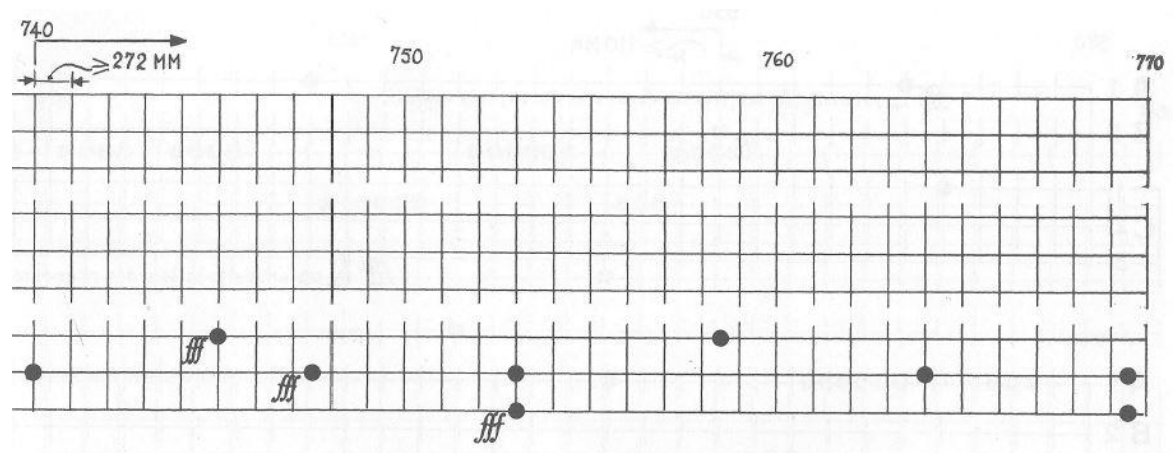

Fig. 3.25 Psappha, mm.740 - 770

Xenakis does not provide phrase markings in the score. The musical relationships between sounds must be at the performer's attention if the work is to become music. A performer may choose to isolate musical phrasing within the group, localizing phrase structure regardless of the happenings in other groups during that time. To portray interweaving of the groups and via musicality between them, the performer may choose to make local musical sense out of the rhythms, regardless of the assigned instrument(s). Figure 3.26 depicts a moment in the piece 
where groups A, B, and C intersect with (a) their own melodic interest that can be played independently or (b) as a melodic composite between the three groups.

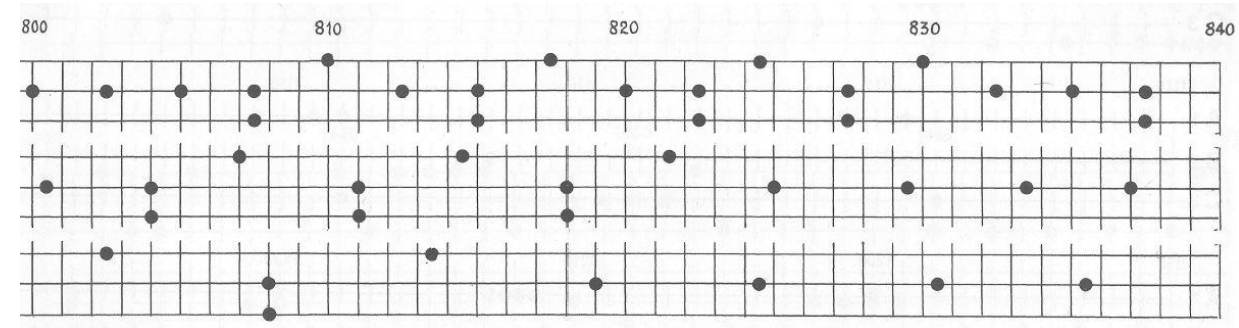

Fig. 3.26 Psappha mm. $800-840$

Xenakis provided standard notation symbols used in the score, another aspect that suggests that he was interested in the playability of the work. The dynamics of the score are explicitly directed in a traditional manner ( $p, m p, m f, f$, and $f f)$, with the sole dynamic changes occurring between MM. $380-518$, which are depicted via the traditional shapes as well as the written "cresc sempre" instruction. Different lines of musical material occasionally have different dynamic marks, which represent a difficult technical task but a notation that is easily perceivable. In these moments, courtesy dynamic markings are provided in parenthesis. The traditional two slashes that accompany select note heads beginning in box 2023 are used to depict "2 or 3 strikes per stroke," a familiar notation despite the serious technical consideration and skill needed to fulfill Xenakis' request.

The only articulation mark used is the accent, whose symbol legibly hovers in the conventional way over the corresponding note-head. If the performer chooses to interpret the accents only as a louder stroke, then this notation presents no advanced problems. However, as Sallak translates, Xenakis models five ways in which the performer can interpret accents: “(1) a louder stroke; (2) a different, but similar instrument; (3) a different stick or mallet, (4) the 
simultaneous striking of two instruments, or (5) any combination thereof." 84 If the performer chooses either of the latter four models, then a learning curve will result from a different translation needing to occur immediately upon seeing the familiar accent symbol. Freedom to act as part-composer is thereby granted on the performer with practically endless permutations, which might manifest in the equipment and setup dimension via instrumentation and/or implement changes, or the technical dimension via louder strikes, harder strikes, and/or doublestops orchestrated between two instruments.

Despite its distinctive notational qualities, Psappha is an intuitive score to read. Instrument assignments and rhythmic relationships are easily distinguishable once a level of literacy is established. Upon re-writing a few short figures into variants of standard notation, I am convinced that the grid score is a much more efficient portrayal of Xenakis' composition. The conventional symbols for dynamics, accents, and sustained rolls recollect previous reading experiences for the percussionist. Although the coordination demands are high, and the material is quite rhythmically dense, I found that reading the score during performance was possible and even preferred over memorization.

${ }^{84}$ Sallak, 55. 


\section{CHAPTER 4: TECHNICAL DEMANDS}

There are a variety of techniques that are needed to traverse the advanced solo multiple percussion literature, with a wide breadth of demands based on variables such as musical style, diversity of instrument material, and size of the setup. Nonetheless, technical skill in the multiple percussion medium should be primary objectives in typical university program curricula within the lens of core instrument instruction. The basic stroke types, proper tone production, and the application of rudiments and stickings are just some of the techniques likely practiced elsewhere that directly apply to multiple percussion. Conversely, technical skills predominantly stressed in multiple percussion also apply to other percussion instrument families, such as four-way limb coordination and independence, handling implement changes and negotiating specified playing spots. Gould recognizes the interrelationship between technical skills and musicianship as he details six "techniques needed to advance musical nuance" in multiple percussion: stroke, sticking, dynamic control, tone production, polyphonic playing and musical gesture. ${ }^{85}$ I present this chapter to describe how the technical dimension poses its own unique set of problems that can be isolated and developed within advanced study of the multiple percussion repertoire.

\section{Dynamic Control}

Dynamic control is a universal percussion technique that can be honed with slight modification for the multiple percussion context. Gould poses two large categories in dynamic control: externalized concepts and internalized technical facility. The former category recognizes that instrument and implement choices have effects on dynamics. The latter category refers to three techniques the percussionist can develop for dynamic control: stroke, playing area, and

\footnotetext{
${ }^{85}$ Michael Gould, "Advanced Multiple Percussion Techniques: An Analysis with Musical Approached to Performance Problems in the Music of David Hollinden," (DMA document: University of Kentucky, 1999), 1.
} 
accent control. ${ }^{86}$ Gould suggests dedicating specific muscle groups to strokes within dynamic bandwidths: fingers for piano, wrist for mezzo, and forearm and shoulder for forte. In relation to playing area, membranophones will have a louder possible dynamic in the center or just-off center. Idiophones will also have ideal playing spots in terms of dynamics. In my perspective, while both aspects contribute to overall volume, muscle group attribution also alters articulation and musical gesture, and playing spot also alters overall timbre. In this way, percussionists should not rely on muscle group or playing area to be the sole arbiter of their dynamic range, but rather should use them in conjunction with stick height, as well as instrument and implement choices, to achieve their desired sound.

Softer dynamics will require further training than louder dynamics, especially for passages that move between surfaces; it is very difficult to quickly traverse a large distance while maintaining a piano dynamic. One consideration is to harness the natural rebound of one stroke to propel the implement horizontally, rather than the typical vertical rebound. One may prepare for this rebound by moving in the direction of the next surface during the previous stroke, similar to how a marching tenor player might prepare a sweep technique. Careful attention should be paid to the overall dynamic across the setup, as instruments will produce varying dynamics.

\section{Stroke Types}

Fluency with the basic stroke types is a requisite skill in the advanced literature. Michael Gordon's $X Y$ (1997) for five tuned drums consists of constant full strokes throughout its fifteenminute length, requiring fluidity, consistency, and comfort from the percussionist. Gradual dynamic changes occur in each hand independently, so that percussionists must apply stick velocity for rhythms to stay consistent regardless of stick height (Fig. 4.1). The absolute mastery

\footnotetext{
${ }^{86}$ Ibid., 107 - 116.
} 
of rebound control and endurance makes the piece an excellent choice for developing this most important technique for percussionists.

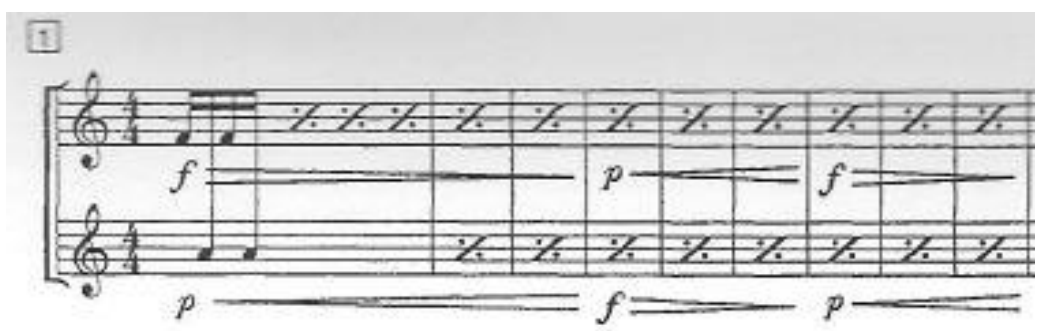

Fig. 4.1 $X Y$, opening

For Xenakis' Rebonds A, up- and down-strokes are emphasized for accents to effectively emerge from the musical texture. Tap strokes are also evident within the texture, notably for those passages where one hand contains a constant rhythmic drone. Although stickings are not specified, an example of one hand executing taps with occasional accents occurs on the high bongo in Rebonds B (Fig. 4.2). Tap and accent control skills may transfer from rudimental drumming to the solo multiple percussion realm, but the latter notably requires further rebound control between surfaces of vastly different material and tension, as well as musical elements such as pitch and timbral difference via a wide orchestration.

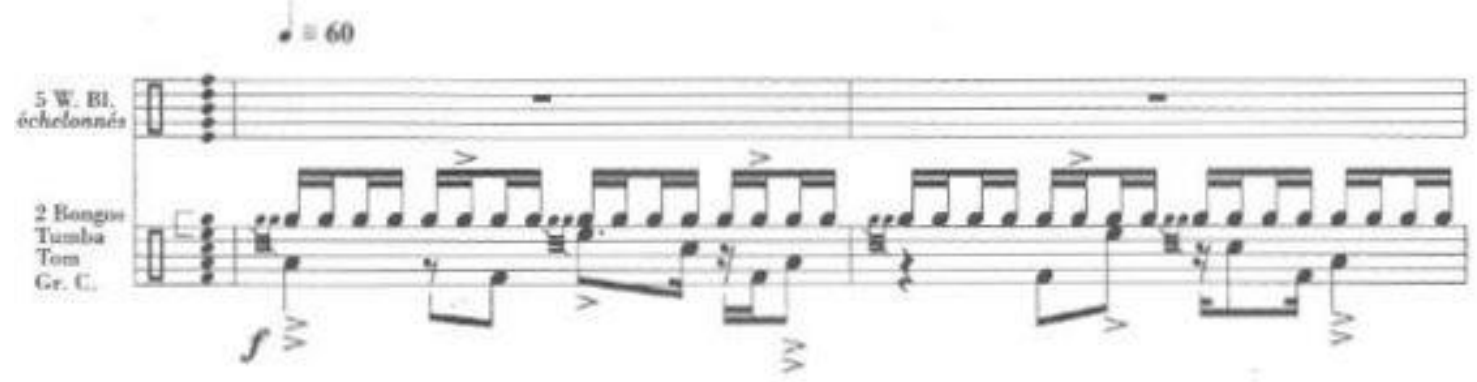

Fig. 4.2 Rebonds $B$, opening

In addition to the basic single stroke types, double-stroke (stroke and bounce) and multiple-stroke types are also significant techniques within the advanced multiple percussion literature. Both stroke types are rarely notated explicitly by the composer, but often result from a performer's choice. For example, double-strokes may eliminate motion between instruments of a 
great distance, improve musical phrasing, or create an expressive gesture. An exceptional instance where the composer prescribes double-strokes appears in Benjamin Finley's Blade (Fig. 4.3).

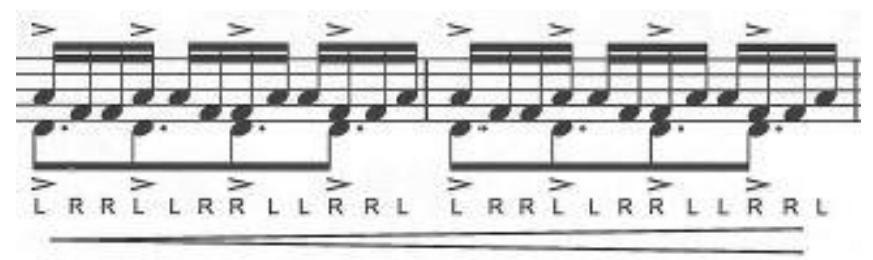

Fig. 4.3 Blade, double-stroke passage

Multiple-bounce rolls are necessary to create a smooth sustained sound on short-sounding instruments, particularly those with a high pitch, and are therefore a requisite skill throughout the repertoire. Joseph Tompkins references Ionisation themes in his solo To Varèse, consequently prescribing the orchestral rolls and single-handed press strokes. Tompkins clearly differentiates between allowing the performer to apply sensitivity to create the sustained sounds here with his explicit specifications for various tuplets and open rolls used elsewhere in the piece. Shin-Ichiro Ikebe's A Snare Growls, and He Flies is a piece for snare drum, snare stand and floor that utilizes exceptionally long orchestral rolls with subtle manipulations that traverse the entire dynamic spectrum (Fig. 4.4).

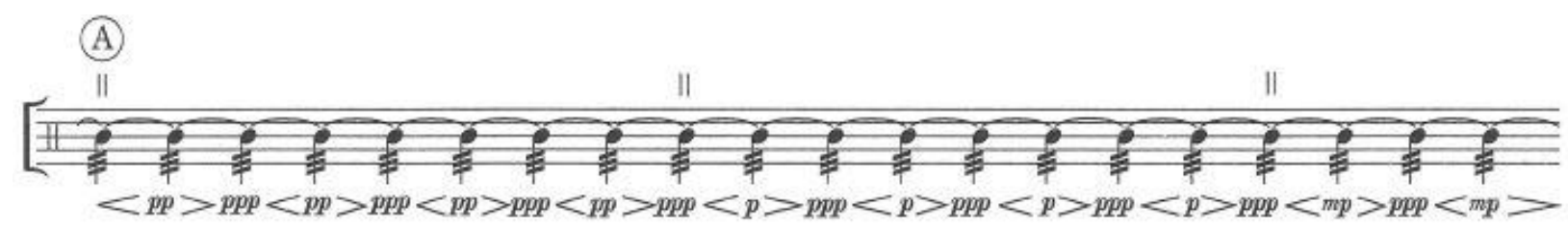

Fig. 4.4 A Snare Growls, and He Flies, multiple-bounce fluency

Gould also describes alterations to the general stroke to develop advanced control and create extended techniques into one's vocabulary. The author describes details of finger control, referring to how digits can either work individually or together as a unit. Gould also describes dead strokes, which can be realized with pressure and weight distribution with the fingers or the 
arm. The dead stroke is an extended technique called on with regularity in multiple percussion (i.e., Thirteen Drums), as well as marimba (i.e., Keiko Abe's Variations on a Japanese Children's Songs), timpani (i.e. Elliot Carter's Improvisation), and other percussion genres. The crawl technique is also detailed, which "moves the pivot point forward after each successive stroke." ${ }^{87}$ Gould articulates how additional arm weight "will add emphasis in tone, add a marcato effect, or help with agogic accent." 88 The author uses these techniques to describe advanced interpretations of two pieces by David Hollinden: Cold Pressed and Six Ideas for Snare, Bass, and Cymbal.

\section{Multiple Stops}

The double-stop is a technique where two or more notes are struck simultaneously, a necessary technique in keyboards and timpani, as well as multiple percussion. In the keyboard and timpani contexts, a double-stop refers to having one implement in each hand strike two surfaces simultaneously to produce a "chord." In multiple percussion, the distance between two instruments are variable. Thereby, "interval” control and playing spot recognition via visual, aural, and kinesthetic means are necessary skills. Instruments may lay at different heights in multiple percussion as well, so the percussionist must be aware of the flight path, stick height, and stick velocity in order to strike the desired surfaces simultaneously.

Advanced techniques are needed to highlight one voice or musical line within a series of double-stops, such as altering playing spot, grip or stick height. In the latter, the primary voice is approached using a higher stick height, in which case velocity must be applied to the stroke to cover the extra distance. Ultimately, all the implements need to strike their respective surfaces

\footnotetext{
${ }^{87}$ Ibid., 82 .
}

${ }^{88}$ Ibid., 83. 
simultaneously. She Who Sleeps with a Small Blanket (1986) by Kevin Volans contains fast passages of double-stops in the "In Speech Rhythm" section (Fig. 4.5)

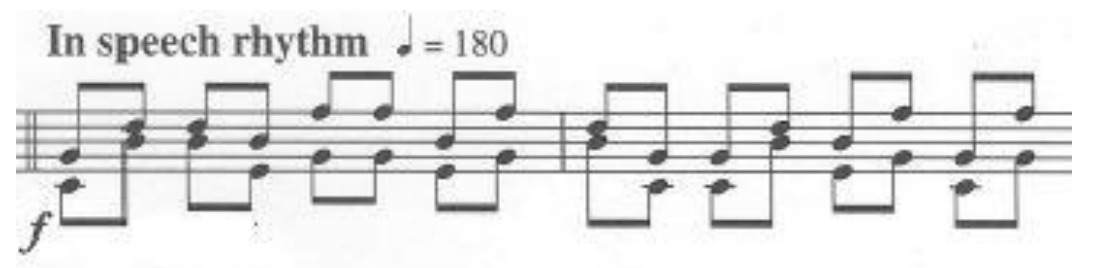

Fig. 4.5 She Who Sleeps with a Small Blanket, double-stop passage

Gould notes that "there are four modes of polyphonic playing which involve from two to five separate lines... two-way, three-way, four-way, and five-way." ${ }^{89}$ While Gould refers to the feet and voice creating the rich polyphonic textures, Inspirations Diabolique, Movt. II. Dance by Ricky Tagawa serves as an example of four voices that result from a four-mallet grip only (Fig. 4.6). Using the voice in conjunction with the limbs poses additional problems, which is discussed in Chapter 5.

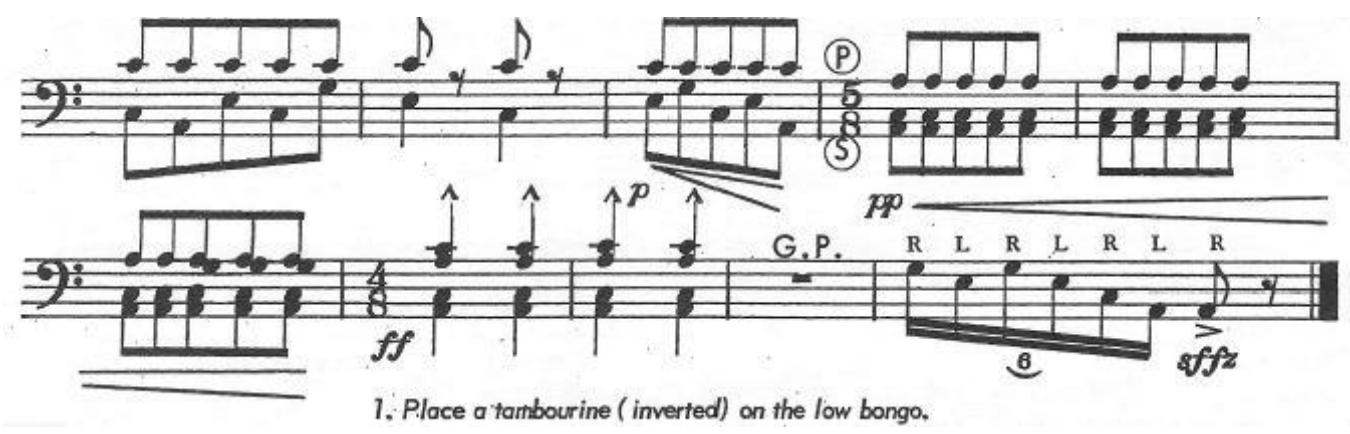

Fig. 4.6 Inspirations Diabolique, Movt. II. Dance, multiple-stop passage

The addition of the feet poses particular challenges for classical percussionists, as those limbs are not responsible for producing sound in the other core instruments. Percussionists would do well to address their foot pedal technique and develop their coordination, evenness, and strength via drum set method books such as Dahlgren and Fine's 4-Way Coordination or Reed's

\footnotetext{
${ }^{89}$ Ibid., 151.
} 
Progressive Steps to Syncopation for the Modern Drummer. Percussionists might choose a heeldown technique, which offers more body support and balance while sacrificing strength and weight of the stroke. The heel-down technique typically has the reverse effect. The detriments of each technique are expounded when the percussionist must remain in a standing position, as is often the case in multiple percussion. Michio Kitazume's Side by Side (1979/2005) and She Who Sleeps with a Small Blanket are excellent introductions to using the feet because only one foot pedal is used and the relatively few notes in that voice only accentuate certain notes already played in the hands.

Both feet performing simultaneously is an additional challenge not only in the precision of their rhythm, dynamic, and tone production, but also overall balance and poise that inherently affects facility of the entire body. The finale of David Lang's The Anvil Chorus requires navigating between five foot pedals, the speed of which will likely influence the percussionist to use both feet (Fig. 4.7). Lang reserves simultaneous strikes with both feet to just one moment in the final full measure of the piece, perhaps recognizing the difficulty of such a technique.

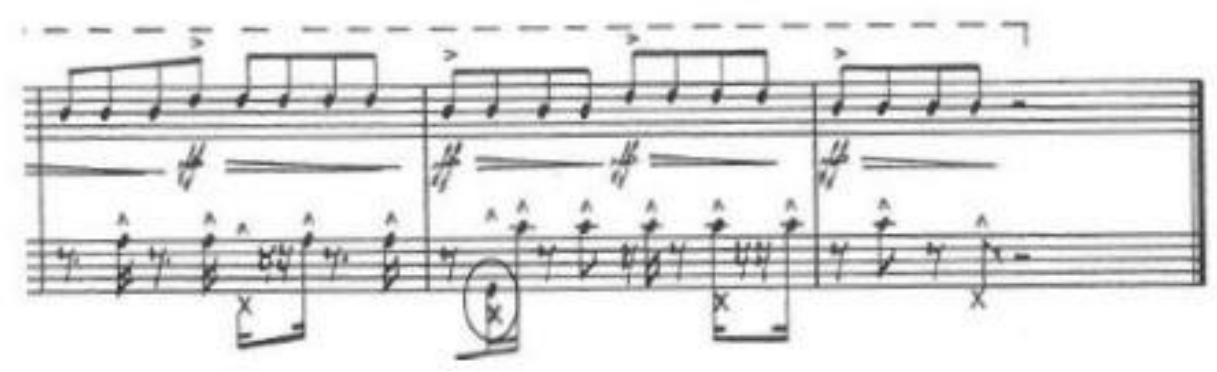

Fig. 4.7 The Anvil Chorus, finale (double-stop in the feet circled)

\section{Coordination and Independence}

As already implied in the following description, limb independence is a crucial part of performing in the multiple percussion medium. Like drum set playing, significant instances in the repertoire call for an isolated limb of the percussionist to be restricted to select instruments. 
This can manifest in a regular pulse or an ostinato pattern in which the percussionist must also provide additional musical voices with their other limb(s). A simple introduction to this technique involves a passage from The Love of L'Histoire where one hand plays straight sixteenth notes on the mounted tambourine while the other hand performs a simple rhythmic counterpoint in the drums (Fig. 4.8).

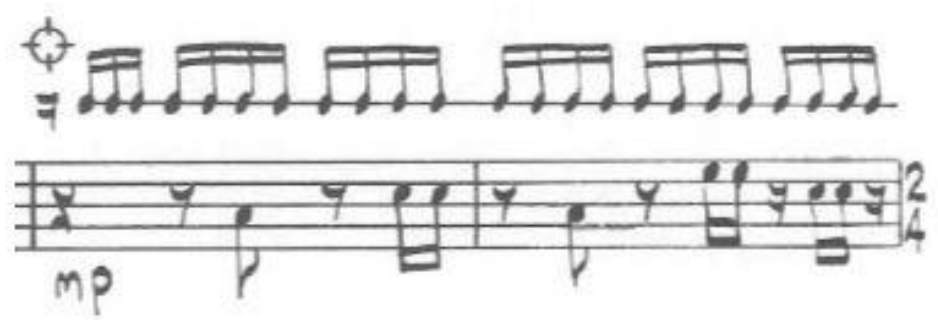

Fig. 4.8 The Love of L'Histoire, tambourine ostinato

Along with other pieces in his offerings, Casey Cangelosi's Evil Ernie contains extensive passages where this type of intermediate independence is highlighted, the first such example containing straight sixteenth notes on the metal plate (Fig. 4.9).${ }^{90}$ Fast interjections of the ostinatos that require both hands and later ostinatos on different instruments make the independence in Evil Ernie a slightly more difficult task.

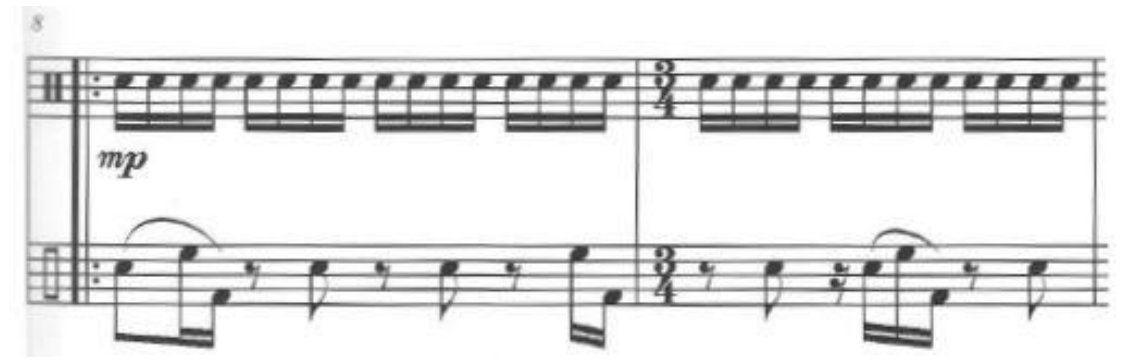

Fig. 4.9 Evil Ernie, simple independence

Increased independence of limbs is needed as one progresses through to the advanced literature. Changing the playing surface of the ostinato without a change of rhythm is a logical progression, such as the straight eighth notes in The Anvil Chorus resonant pipe theme (Fig. 4.7,

${ }^{90}$ Casey Cangelosi, Evil Ernie (Princeton, WV: Cangelosi Publications, 2009). 
top stave). Another progressively difficult task to independence utilizes accents, such as those found in Rebonds B (Fig. 4.2). To Varèse by Joseph Tompkins incorporates an ostinato in the feet, resembling a typical drum set pattern, underneath longer and more varied patterns played with the hands (Fig. 4.10). The piece requires the nondominant foot to change from foot-cabasa to the mounted tambourine, a small technical task that needs to be developed in order to execute the foot pedal work of The Anvil Chorus.

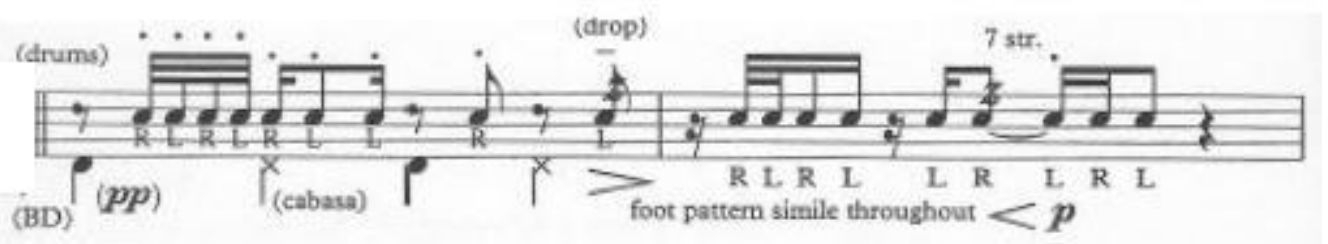

Fig. 4.10 To Varèse, feet ostinato

The advanced repertoire tends to utilize longer patterns and more difficult polyrhythms that challenge the percussionist's coordination and independence. The percussion curriculum may serve to isolate the two different planes in which independence can manifest: (1) the horizontal plane, which equates to changing surfaces, and (2) the vertical plane, where simultaneous polyrhythmic independence is needed. Changing playing surfaces is a stark challenge to the finale of Rebonds B, where the orchestration of five woodblocks over the four drums creates a lengthy and coordinated pattern, even though the vertical rhythm between the hands is the simplest 1:1 ratio (Fig. 4.11).

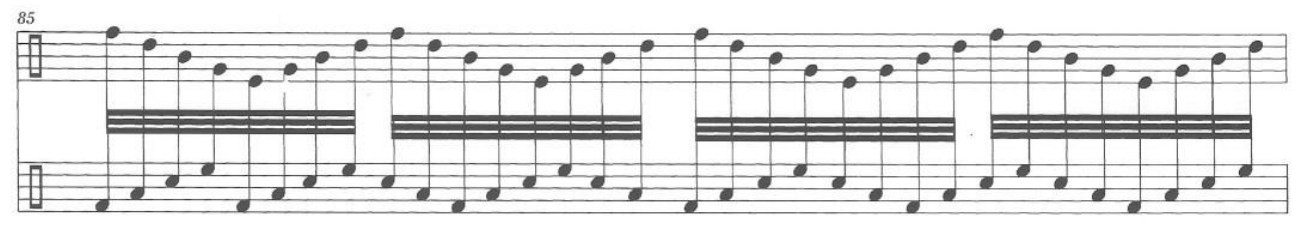

Fig. 4.11 Rebonds B, advanced orchestration independence

Contrastingly, $X Y$ presents numerous difficult passages in the vertical plane, even though the orchestration is relatively simple. The section beginning with measure 413 marks four-note 
patterns in both hands, but one hand playing sextuplets while the other playing quintuplets (Fig. 4.12). An additional vertical problem in this work is the independent treatments of dynamics between the hands, often reverse of each other. The three different treatment of dynamics for each of the three repetitions of the 8-bar section demands great independence in coordination.

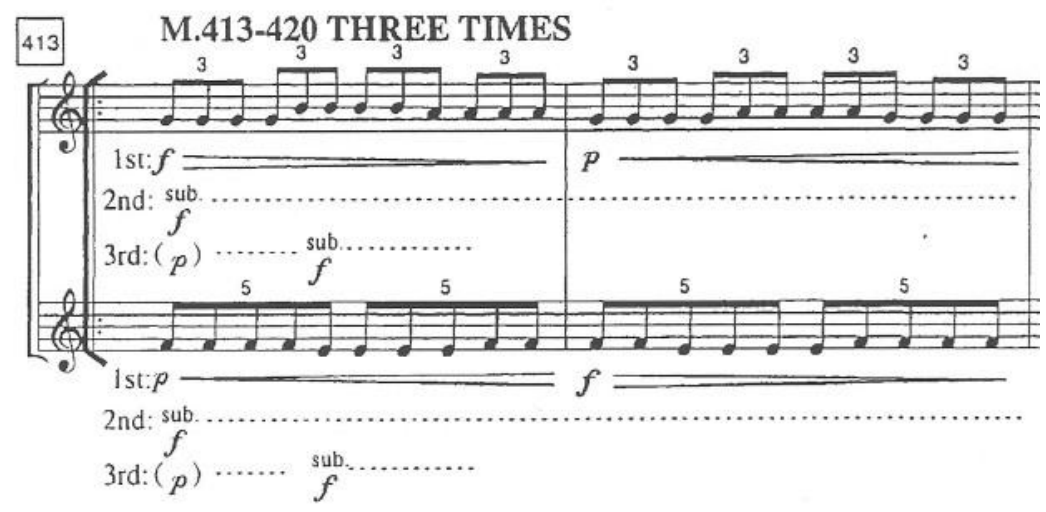

Fig. 4.12 XY, advanced polyrhythmic independence

\section{Four-Mallet Grip}

Although typically considered a technique within marimba and vibraphone curricula, fluency in a four-mallet grip is pivotal for the advanced multiple percussion literature as well. Each grip has their advantages and disadvantages; interested readers should consult Glassock for comparison of the grips. ${ }^{91}$ As described by Nancy Zeltsman, the natural leverage and power awarded, being able to grasp the mallets anywhere on the shafts, and the ability for quick mallet changes are all benefits to the multiple percussionist. ${ }^{92}$ Multiple percussion solos that require one hand to hold two mallets simultaneously include She Who Sleeps with a Small Blanket, Rogośānti (1986), and Psappha. In one particular passage of Psappha (Fig. 4.13), one hand must navigate between four metal instruments quickly, an exceptional case where the importance of independent interval changes would make the Stevens grip advantageous.

\footnotetext{
${ }^{91}$ Lynn Glassock, "Four-Mallet Grips," Percussionist 11 (1973): 6.

92 Nancy Zeltsman, “Traditional Four-Mallet Grip,” Percussive Notes 33, no. 4 (1995): 54.
} 


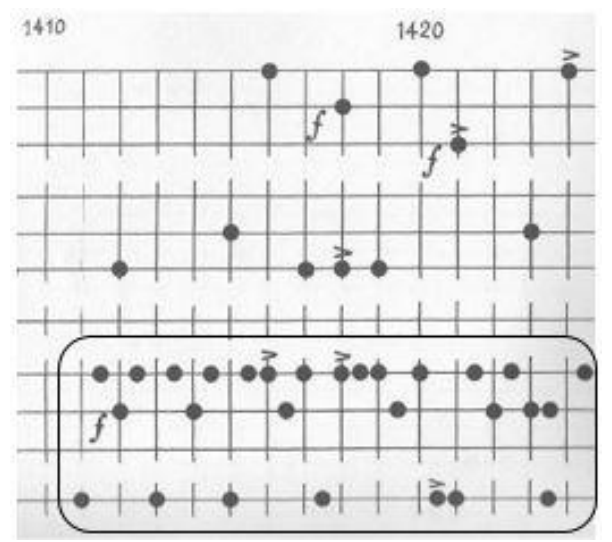

Fig. 4.13 Psappha, excerpt utilizing 4-mallets

While decisions concerning the choice of implements falls into the purview of the equipment and setup dimension, the coordination of physically making implement changes is a technical facility. Independence control is necessary to change one implement while the other continues playing with precise rhythm and a consistent playing spot, which manifests throughout Stockhausen's Zyklus, among other works. Percussionists need to develop their ability to quietly and accurately place the implement on the trap stand, especially if that implement is needed again later in the piece. Regardless of grip, percussionists may find switching implements an intricate procedure in a four-mallet grip. Depending on which mallet needs to be switched, it is often easier to drop both mallets and pick up the desired mallets from a neutral position. Without the practice of precise choreography, implement changes might ill-affect musical flow and treatment.

\section{Motion and Gesture}

Movement in general is an idiom with discourse within both technical and conceptual dimensions. Technical examination encompasses the motion between instruments as a job that requires certain tasks to be executed properly, while the conceptual might examine how movement may be utilized, exaggerated, or added to moments to provide drama to a musical performance. 
Motion between instruments is a significant technique that is influenced by the equipment and setup dimension, as well. The size of the instrumentation and the setup design have direct implications to the difficulty of technical movement. The concept of shifting is one that can be developed within the multiple percussion repertoire. The most efficient movement between different surfaces includes a stroke that rebounds in the direction of the next instrument for that particular limb. One might even attempt to rebound directly over the next surface to be struck at the height to produce the correct dynamic. The percussionist's efficiency of movement and quality shifting is tested in Stonewave, which requires traversing the entire massive setup (Fig. 2.7) with thirty-second note and indeterminate flourishes, with one passage quickly traveling between stations: from Drum Setup 2 to Drum Setup 1 and finally to the Metal group (Fig. 4.14).

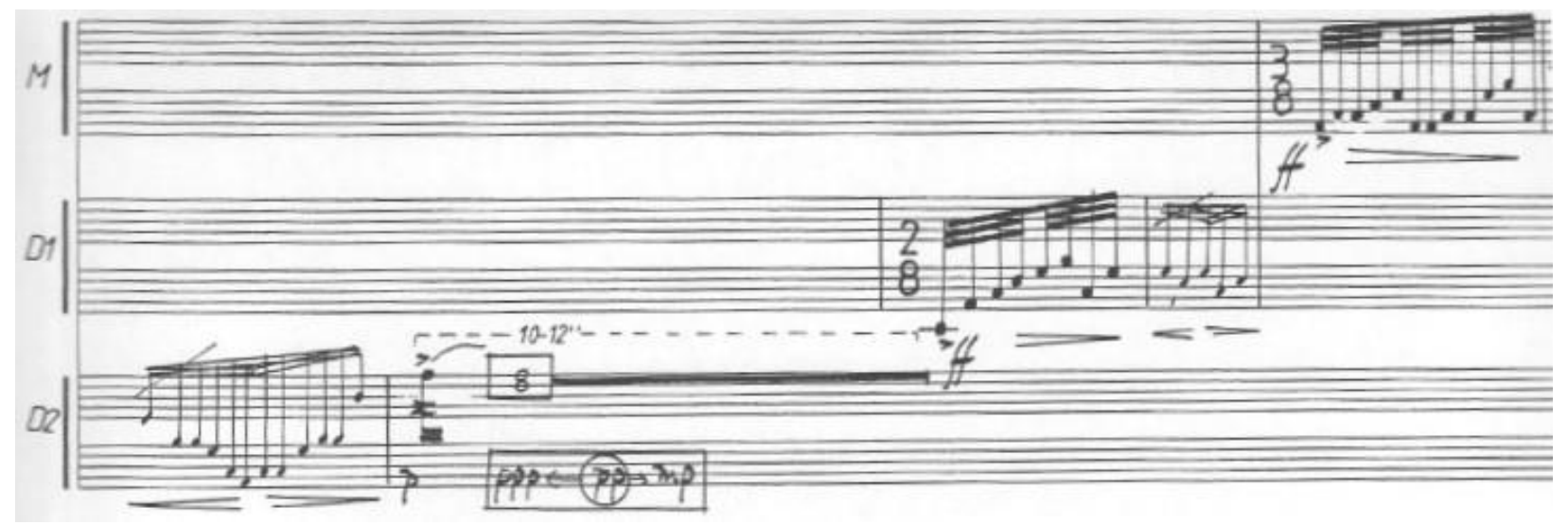

Fig. 4.14 Stonewave, quick motion across large set-up

Movement and gestures should also be considered within the context of instrumentation. Smaller instrumentations require gestures to become smaller and more subtle if they are to be perceived appropriately. Instruments of little resonance will similarly take smaller gestures, and a percussionist should avoid a disconnect that would result from a grand gesture that produces a small, short, or thin sound. The opposite is also true, as gestures need to be exaggerated if the audience is going to see them over a bulky setup. Setup design also plays a role in gestures 
because the flight paths of the sticks/mallets need to be clear in order to execute a gesture with virtuosity; the percussionist cannot miss the target area or graze another instrument in their strokes, and the best performances have no fear of such occurrences.

As a technique, a gesture is the distribution of the percussionist's energy to a larger muscle group (forearm, upper arm) to execute a note or passage. Schick describes the basis of a general gesture technique in percussion:

"Most percussive gesture consists of two concurrent bodily tempi that are typified by two very different types of motion: fast movements necessary to playing individual notes are usually angular motions, but slower preparatory movements involving ball-and-socket joints are often curving., 93

In my perspective, the larger muscle group inherently moves slower than smaller muscle groups (fingers, wrist), so a gesture implies that the motion will be more fluid and grander. A gesture may service the musical phrasing by placing more weight onto a note, allowing for the musical attribute of tenuto. A gesture may also serve the purpose of creating more torque at the onset of a double-, triple-, or multiple-bounce stroke. The torque that is created with the large muscles can then be manipulated by the smaller muscle groups (wrist, fingers) to execute subsequent notes. A common school of practice utilizes the same process for the multiple-bounce stroke, in which the initial movement comes from the arm. These advanced techniques can aid in the realization of multiple percussion scores and provide further articulation vocabulary for musical interpretations, which can be developed in multiple percussion or any other core instrument curricula. While the technical aspect of motion and gesture is necessary for all professional percussionists, its development within the multiple percussion medium can incorporate variables of instrumentation sizes and setup design, as well as fluency of all axes: $x$,

\footnotetext{
${ }^{93}$ Schick, 142.
} 
$y$, and $z$. Of course, dramatic elements can be created via the performer's motion and gesture, which will be discussed in Chapter 5 .

Maki Ishii, Thirteen Drums (1985)

Performed on April 8, 2017, DMA Recital in Falbo Theater, West Virginia University. A percussionist aspiring to perform Maki Ishii's Thirteen Drums (1985) will encounter several technical challenges. The instrumentation is fairly straight forward: thirteen drums, one being a bass drum operated by a foot pedal. Charles found the piece to be "one of the more athletic pieces...requiring the performer to execute rapid passages across a massive span of drums. ${ }^{" 94}$ Instrument design is significant to overall movement, stickings and possible tempo, especially considering rapid passages and flourishes. However, a single ideal setup is rendered impossible because of Ishii's nuance in orchestration and performer preferences. Positioning the smallest, highest-pitched drums closest to me was a logical choice that created a consolidated setup and minimized the span needed for outward motion (Fig. 4.15). Placing the lowest, longest instruments farthest away from the performer makes the physical gestures required to access them match the sound they produce.

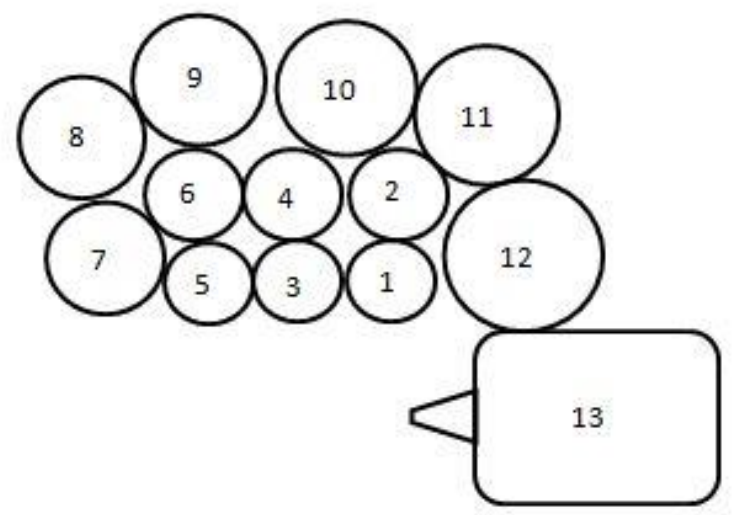

Fig. 4.15 Thirteen Drums, Greco setup design

${ }^{94}$ Charles, 44 - 45. 
While this setup had the advantage of instrument proximity, it did impose challenges. The highest six drums descend counter-intuitively in two rows, moving "up" and then right to left. To make matters worse, the progression switches from drums 7 to 12 , moving in one row from left to right. Another challenge in navigating the setup and score relationship emerges from their divergence in distance. For example, drums 1 and 12 are physically very close to each other in my setup, while drums 7 and 11 are quite far. However, those relationships are reversed on the score. All the notes for the bass drum in the right of the setup were played by my right foot. A more logical position may have been in the center of the setup, so that the notes could be distributed among the feet when appropriate. However, I did not have access to the necessary hardware at the time I was learning the piece. Using the right foot was more natural, given my experience with drum set. Truthfully, this nonlinear setup did cause a learning curve in reading the score, although it proved advantageous technically. Interested readers should consult Berry's description of three different instrument designs and their performance implications for the space-time concept. ${ }^{95}$

There are two main types of rhythms in Thirteen Drums: determinant rhythms that are precisely notated and indeterminate grace-note rhythms that are left to the performer to fully realize. Before exploring the advanced techniques for the indeterminate gestures, I will firstly address techniques for the determinant passages in the piece. Paramount is an absolute command of single alternating strokes. For speed and gesture, single alternating strokes are highly encouraged for the piece's opening figure (Fig. 4.16). One may choose to give a slight agogic accent in the four-note groupings in this gesture, or to play perfectly even. No matter which interpretation is chosen, the consistency of phrasing and articulation is key throughout the

\footnotetext{
${ }^{95}$ Mark Berry, "Thirteen Drums for Percussion Solo, op. 66: Interpreting in Concurrence with Make Ishii's 'SpaceTime' Concept," Percussion Notes 47, no. 5 (2009): 50 - 59.
} 
dynamic change. One could adopt Gould's crawl concept and move their fulcrum forward throughout the diminuendo, especially toward the end of the fade. This same figure occurs on each of the odd-numbered drums $(5,3,9,1,11,7)$, and each undoubtedly will have different physical and sonic responses. When striking the lower drums that are looser and offer less natural rebound, one might bring their middle finger slightly more around the stick and increase its contribution of energy to putting the stick in motion.

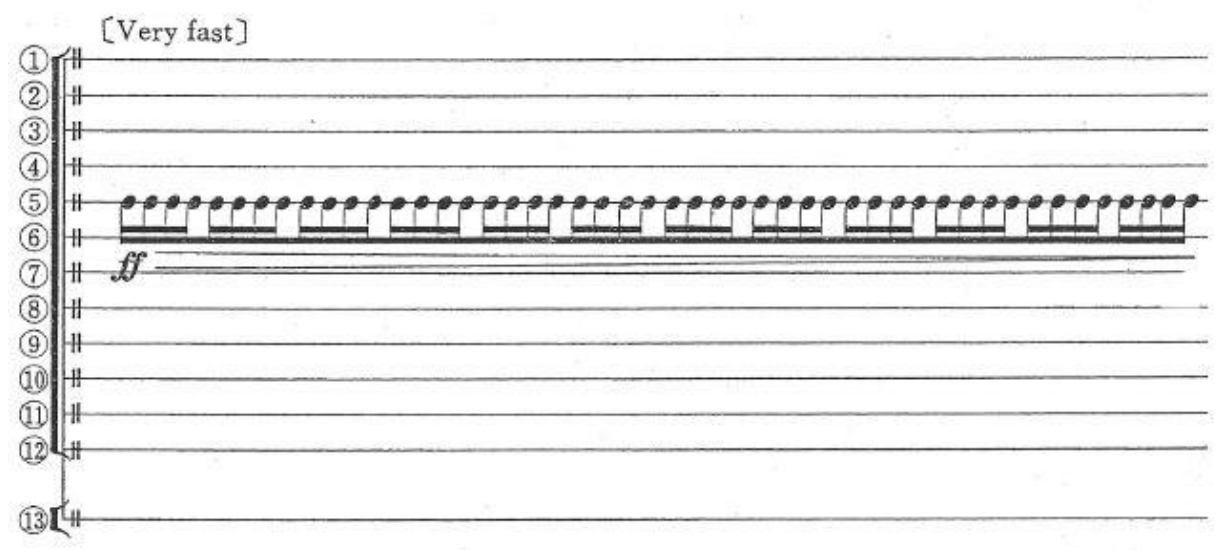

Fig. 4.16 Thirteen Drums, opening figure

Beginning in the fourth figure of determinant rhythm, Ishii orchestrates select sixteenth notes on other drums, forming a melodic phrase that will be repeated and expanded on throughout the piece. The melody expands from its first three-note statement on drums 5-3-11 to the final statement of the section, which traverses each of the odd-numbered drums (minus the bass drum) 5-3-11-1-9-7-5-7-9-1-11-3-5, the middle 5 acting as the initiation of the phrase's retrograde. Consistency is key in this section not only to create fluidity in the melody, but also in tone production to produce a drone effect on the stationary drum. The playing spot on the drone drum must be maintained while the player traverses the other drums in the setup.

Sticking is a primary issue for this section, of which there are three general solutions (Fig. 4.17). Depending on one's setup and preferences, all provided stickings may be reversed. To construct uniformity between the two gestures, percussionists may choose to maintain the 
alternating sticking from the diminuendo figure. Both hands will be required to move to the melodic drums depending on the partial of the beat on which the note occurs. The hand that travels will consistently have one eighth note to do so, a welcomed expectation. Yet, a challenge arises because there are varying distances needed to travel between the drums in that same amount of time. Since the percussionist performs the drone on each even-numbered drum within the sequence 8-4-10-6-2-12, those who choose an alternate sticking will need to make use of cross-sticking and "under-stickings" to strike the melodic drums. Cross-stickings need to be aware of where a cross is positioned on the arm/wrist to enable proper motion, as well as the angle that the mallet-head/stick-bead strikes the surface for proper tone production. Understickings require the arm of the droning hand to swing out, providing access to a drum that is positioned closer to the body. Cross- and under-sticking can be avoided if the percussionist orchestrates select double stickings. By doubling on the droning drum immediately preceding melodic notes when necessary, each hand can be accorded intuitive movement that is free of obstruction. When the double occurs, the stick is also given more time to travel: the equivalent to a dotted-eighth note. The doubles need to sound just like the alternating sticking, which I found to be especially difficult because of their infrequent occurrence. Preparation is key to achieve consistency. 


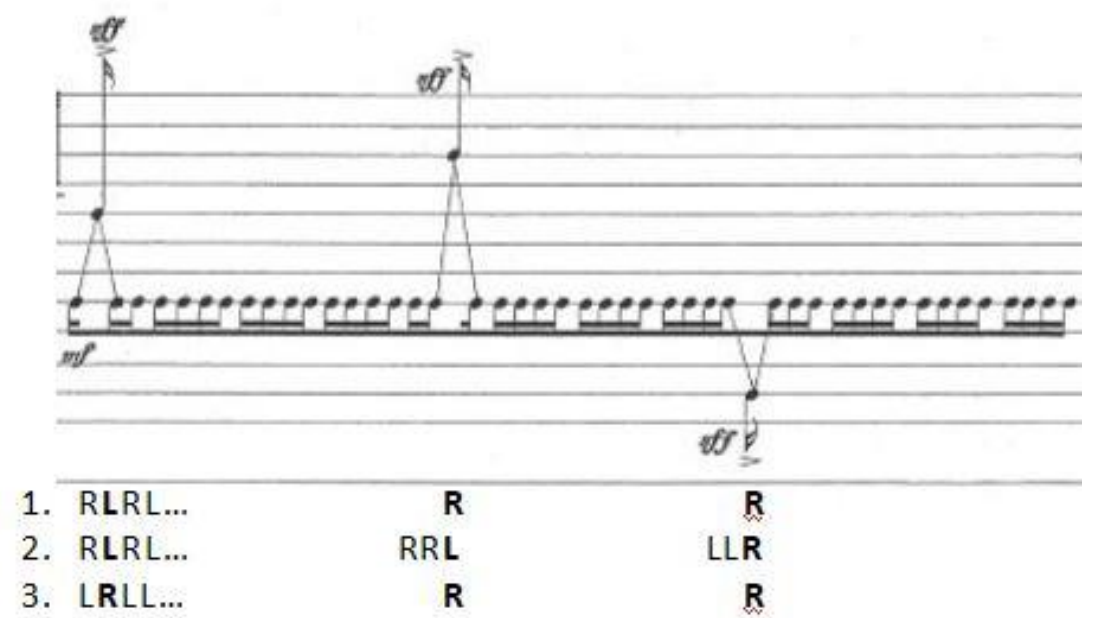

Fig. 4.17 Thirteen Drums, first melodic phrase with three sticking models

Ultimately, I chose to regulate each hand to either the drone or the melody for individual figures in this section. I employed either the left or right stick to perform taps on the stationary drum, depending on the location of the drum in my setup. Performing single-hand taps was challenging to reach and maintain the desired speed in each passage, especially for the nondominant hand. I sought to use primarily finger motion to help to facilitate speed and ease. A technique that uses the arm without raising the mallet-head/stick-bead is also helpful to create torque that can be applied to future strikes without changing dynamics. Drum set players may find familiarity with these techniques in their dominant hand ride and hi hat figures. To help build my chops in the nondominant hand, I practiced exercises and grooves with an open stance on the drum set, placing my nondominant hand on the hi hat while using the other limbs to help push along the tempo. I would occasionally switch back to the more comfortable right-over-left position to compare the sound and feel.

A common detriment of using finger motion is the relatively thin sound produced because the stroke is not being supported by weight (forearm, upper arm, shoulders). However, this was little consequence given the lower dynamic (mf) and its secondary interest in comparison to the melody. A keen advantage is the consistency that results from using one stick 
for each voice; subtle differences in grip, weight of the stroke, and playing spot, as well as mallet/stick attributes, are not at play. The single-handed sticking gave me an exorbitant amount of time to navigate the melodic notes with one hand, a technique I used primarily for musicality and expression: a grandiose physical gesture will highlight the significance of the melody and can help embody fluidity and connection between the melodic notes, despite long durations between their soundings. Upon developing the taps technique and trusting my kinesthetic and aural awareness to maintain a consistent sound, I used my visual awareness to increase my playing spot accuracy on the melodic drums.

The following section, "molto accento," requires further demands on the player's technical facility. The new melodic phrase experiences ornamentation and rhythmic displacement with each statement, prompting sticking changes as the section progresses. Drum 5 serves as the sixteenth-note rhythmic drone and begins the section by sounding simultaneously with the melodic notes. Accents on both melodic drums and drum 5 are called for in the first two statements, executed by an up-and-down stroke in sixteenth-note succession. I called on a Moeller technique for these notes and many others like it throughout the piece, where upward movement with the elbow brings the stick down for the note prior to an accent. This is followed by the wrist whipping up as the elbow then moves down. Lastly the wrist falls from a high height for an accented sound.

The precisely choreographed double-stop technique on drum 5 slowly gives way to grace notes that ornament the melodic notes from other drums. Attention must be paid to the exact timing of the grace notes, striving for consistency regardless of distance traveled. Since their sound activates and dies out quicker, an advanced technique may put those grace notes on higher-register drums slightly closer to the primary note. An audience may not hear the grace 
notes exactly as they sound or feel from the player's perspective, in which recording yourself in the performance space is an extremely valuable enterprise. In my opinion, grace notes among lower drums should not be so far that they sound like a rhythm, rather the same quality should be maintained throughout the setup. Conscientious movement must take place when the two grace note figures appear, especially since I decided to single stroke all of them. If the grace notes are to be lower in volume than the primary notes, then they must be approached by down strokes in the vertical axis, yet full or up strokes in the horizontal or outward/inward axis, depending on setup. The movements must also be made easy and flowing if the marked accelerando is to be achieved. Excess tension and trying to get it right will not help one perform this passage. I suggest practicing and becoming comfortable with the grace note and primary note figures in isolation without the droning drum 5, which will illuminate the necessary motions and their melodic component without distraction.

The performer must also navigate creating rolls within different contexts in the piece. All rolls contain slashes across the stem, or below the note-head for notes without stems. Some rolls also contain a wavy line that starts at the note-head and proceeds to the right for the note's duration. While this symbol typically indicated a trill in the baroque music, it is doubtful that Ishii desired a distinction between the two symbols at all. Instead, the wavy line appears to indicate longer rolls that endure a change of dynamic or that other notes are to be played while the roll sustains through. The performer is therefore free to realize the rolls as they see fit. In my interpretation, single-stroke, double-stroke, and multiple-bounce stroke rolls were orchestrated to support my musical interpretations of specific sections and passages.

A performer might choose a single-stroke roll for a variety of reasons, in which I found two interpretive advantages in my realization of Thirteen Drums. First, if the sustain involves 
two or more instruments, alternating single strokes helps smooth out the sounds and creates the effect of both sounds melding into one. The second factor comes from a choice to borrow from musical and percussive traits common to Japan. Single-stroke is the primary roll technique in traditional forms of Japanese music in theater contexts, gagaku court music and the more recent virtuosic drumming displays in kumi-daiko. In these genres, percussionists use single strokes to provide larger movement and choreography, an equally important facet to the performance.

The technique also facilitates the manipulation of the speed of the roll, an important facet to exploring Japanese musical traits. One such trait is the concept of $m a$, described by Kite as "the Japanese concept of the fullness of silence and space." 96 Those acquainted with Japanese and select other Asian theater-music styles will recognize regular pulsating percussion that speeds up for increased drama. Ishii explicitly notates a speed change during a roll in select passages (Fig. 4.18), but the concept can appropriately be applied elsewhere as well. Although gradually changing tempo in a single stroke roll is relatively easy, it has limitations. Depending on the size and tautness of the drum, the single stroke may always sound pressing and agitated, similar to when stringed instruments produce a tremolo. Of course, if one slows too much, then the listener will not perceive the sustain. Rapid single-stroke rolls are generally difficult to perform in either extremely soft or loud dynamics. The maximum possible speed will differ depending on the facility of the performer and their chosen instrument, but a move to the doublebounce and subsequently to the multiple-bounce stroke will extend one's speed parameter. I utilized rolls that traveled from single-stroke to multiple-bounce (and vice versa) in select passages of the piece. When transitioning from one stroke to another during a roll, I utilized double-strokes for a split moment to help with the transition.

\footnotetext{
${ }^{96}$ Rebecca Kite, Keiko Abe: A Virtuosic Life (Leesburg, VA: Alfred Music, 2014): 203.
} 


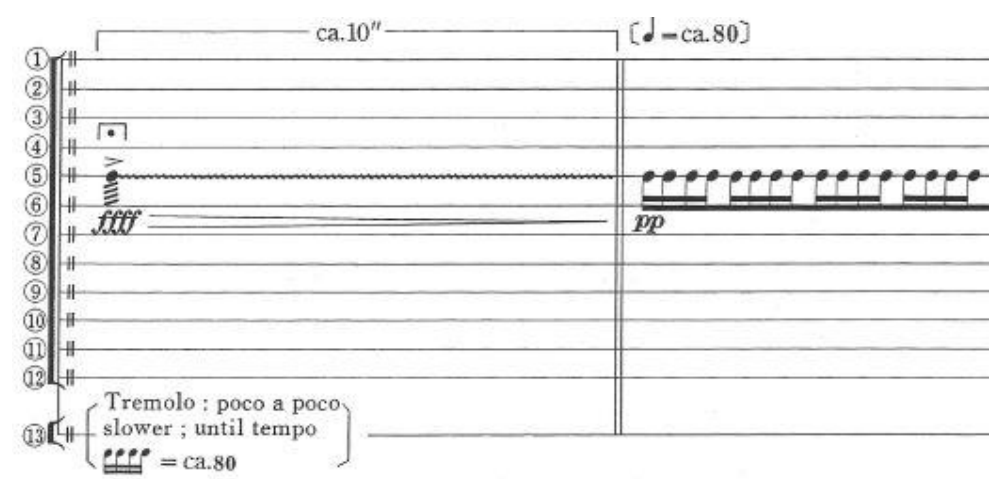

Fig. 4.18 Thirteen Drums, roll speed variation

Smooth and inconspicuous rolls are better executed with a multiple-bounce technique.

Due to the wide range of drums and dynamics called for throughout the piece, the multiplebounce rolls might be as open as a triple-stroke or as closed as completely choked/buzzed. I typically started realizing a passage using the triple-stroke roll in the lowest drums and progressively used more weight from my arm to close the roll as the register of the drums rose. The smoothness of a multiple-bounce roll is particularly advantageous when there is more important material occurring elsewhere. Whereas a single-stroke roll would cover up the sound and visually blur the importance of melodic notes orchestrated during the roll, a multiple-bounce roll allows the sonic space for the articulate color to be heard over the sustained drum. The visual divergence that results from performing a single-stroke on a different drum during a multiplebounce roll also helps the audience conceptualize the two as having separate musical functions.

Thirteen Drums calls for several extended techniques, which ultimately provide very significant timbral changes considering the homogenous instrumentation. Ishii indicates that a note-head with an " $\mathrm{x}$ " through it is to be played "without resound," an interesting vernacular that I interpreted as meaning a dead stroke. I aimed all of the dead strokes for the very middle of the drums, emphasizing the articulation and lessening the resonance. A dead stroke is also called for in this passage on the bass drum, in which I carefully dropped the weight of my leg, pressing the beater into the bass drum while still maintaining the pianissimo dynamic. At a lower dynamic, 
the dead stroke is performed most easily by simply closing the hand, allowing the fingers to provide the motion and restricting the mallet/stick's rebound. The dead stroke technique will require grip and motion adjustment as the tempo and dynamic increases in these passages, requiring more arm weight to get more sound.

There is only one passage that explicitly directs the percussionist to change implements. After indicating the first passage to be played with fingers, Ishii indicates a tied (fluid) roll that melodically moves from drums 5 - through - 9 to be played "with mallet." I speculate that Ishii desires a yarn or felt mallet here. The percussionist has likely chosen to play most of the piece with sticks, or perhaps a wooden mallet, considering the overall dynamic, density, tempo, and energy required. I suggest a timpani mallet to provide a distinct timbre. To help with fluidity in tied rolls, I altered the roll speed so that I had the correct hand responsible for traversing to the next drum first. The percussionist must be fluent and comfortable with odd-numbered groupings to produce the articulation and phrasing desired, regardless of which hand starts the roll or the roll speed. Traveling across an individual drum head during a roll to position the mallet heads closer to the next drum is another advanced technique that emphasizes the fluidity of such lyric passages.

A driving force in the realization of Thirteen Drums is the treatment of indeterminate rhythms. In effect, the exact placement of the grace notes in the "molto accento" section, described previously, may serve as an introduction to them. Berry describes how one's interpretation of indeterminate rhythms is paramount in a successful portrayal of the space-time concept. ${ }^{97}$ The author uses the grace note grouping in the opening sixteenth-note section to warn against two potential misinterpretations: aural inversion and rhythmic distortion. An aural

\footnotetext{
${ }^{97}$ Berry, "Thirteen Drums," 50 - 51.
} 
inversion would perform the grace notes louder than the sixteenth-notes, while a rhythmically distorted performance of the figure would do so unevenly. Both manifestations would be counter to how the figures are notated in the score. Berry analyzes the notation to lead to his interpretation:

"From a visual perspective, the grace notes look lighter and have smaller noteheads, thinner stems, and thinner beams. This would imply a faster flourish of notes. Three slashed beams are used, again implying a quicker gesture than the surrounding sixteenth notes." 98

These detailed parameters by Berry may mean reading the notation a bit too closely for some musicians' taste, as the performer is subsequently restricted to interpret only tempo. One may also notice that the first grace note is marked sff, which is the loudest dynamic marking up to that point. While the notes certainly appear evenly spaced, one must question whether that was the publisher's choice to make the score look neat and clean, rather than the composer's choice to influence a performer's musicianship. Despite this difference in opinion, Berry does mention the paramount consideration in my interpretation: gesture.

A gesture is one motion with the body that has a natural preparation, action, and release. A natural gesture follows an organic rhythm of the body, such as the human lung's inhale and exhale. Any number of actions can be taken during this gesture, like how a wind player may perform many notes with one breath. Similarly, the dancer has numerous combinations that manipulate their upper body while they prep a jump, are actually off the ground, and finally land. Japanese taiko drumming gestures include the preparation, performance, and rebound of a stroke, and this choreography directly applies to Thirteen Drums.

The grouping of the indeterminate rhythms implies a gesture. The size of the note-heads, stems, and number of slashes are not irrelevant, but those notions should be subservient to the

\footnotetext{
${ }^{98}$ Ibid., 51.
} 
gesture. The percussionist has one arm motion in which to hit all the notes prescribed in indeterminate groupings. Those notes can also be manipulated linearly, they can crescendo or diminuendo, but not both. Separately, the notes can accelerando or ritardando, but not both. As long as the player maintains a connection with the manipulation, it will be perceived as one gesture. For the first gesture, I decided to accelerando and diminuendo. Sound waves produced on a low-pitched instrument need slightly more time to develop, causing me to start the gesture slower and then accelerando. The accelerando also propels the gesture forward and gives the appearance of "very fast," regardless of actual tempo.

Maki Ishii's Thirteen Drums is a technical tour-de-force. Since descriptive tempo markings are given, the performer may never fully realize the piece to its completion. Several different kinds of strokes are involved in its performance, and seamless transitions between stroke types give the rolls life and effectively incorporates the Japanese concept of ma. Physical motion and endurance are challenged throughout the bombastic work, while sensitivity and grace in sections will provide contrast for an effective performance. Kinesthetic awareness to gesture will grant the performer expressive context in which to manipulate indeterminate rhythms. The figures discussed within Thirteen Drums provide examples of how technical facility is intertwined with the other performance dimensions in multiple percussion. 


\section{CHAPTER 5: CONCEPTUAL PERFORMANCE}

The conceptual performance dimension consists of various means of presenting formal markers, expressive interpretations and extramusical features, which encapsulates a significant aspect of the contemporary multiple percussion practice. Mid- $20^{\text {th }}$-century artistic circles were attracted to the holistic elements of artistic performance, such as its intersection with dance and movement, the innately primordial act of striking (among other actions) objects, and an emphasis on timbre and rhythm, all of which marked a definitive break from the Classical-Romantic conventions of Western musical performance. Fluxus and the early minimalists are examples of holistic art explorations, now most commonly generalized as early innovators of performance art. We can trace certain ideas and beliefs to John Cage, and even the Dadaists and Futurists that came before, all of which ultimately suggests that the foundation of the Western percussive art form was set by those that were not really percussionists, per se, but artists that blended theater, dance, and music, among others. By surveying the breadth of multiple percussion solo repertoire, with hindsight we see that individual works range stylistically from musical theater to modernism, experimentalism to minimalism and everything in between. The refinement of percussive expertise is simply assumed for the working percussionist today, but it may be the student's ability to navigate conceptual elements, moving beyond those instructions that are purely sound, that are requisite for their success in multiple percussion.

Strom suggests the term theater percussion be applied to the subgenre that combines percussion with extramusical components, a style of music that "has been alive since the midtwentieth century." ${ }^{99}$ She provides a short sample list of relevant theater percussion works

\footnotetext{
${ }^{99}$ Julie Strom, "Theater Percussion: Developing a Twenty-first-century Genre Through the Connection of Visual, Dramatic, and Percussive Arts," (DMA document: University of Northern Colorado, 2012), 5 - 6.
} 
totaling forty-four pieces, a number of which are no longer in print or were never published. ${ }^{100}$ In addition to a subgenre that encompasses a wide range of styles, we may notice that it presents any number of demands in the other dimensions of multiple percussion. Equipment and setup, notation, and technical facility will always be integral aspects of these works, and while conceptual performance is interrelated to these dimensions, certain demands require distinct skills and concepts.

While Strom's focus is on identifying and naming the genre and the validity of doing so, her findings may apply to our focus of percussion education as well. The author argues that "labeling a style of music encourages composers, audiences, and musicians alike to compose, perform, and listen to theater percussion - an alternative form of purchasing a product." ${ }^{101}$ In addition to encouraging its survival and marketability to the different "consumers" of theater percussion, Strom also suggests that creating the label will allow audiences to categorize as well as ultimately critique and evaluate these works. If one is to believe in an audience's ability to evaluate, the highest Bloom taxonomy, then performers would certainly be able to accomplish less complex objectives: to synthesize performance skills and appropriately apply them to other works they perform in the future. Considering elements are widespread in the growing repertoire, skills developed in the realization of one piece will transfer to the next, and consequently, a curriculum can be posited that would prove beneficial for both instructor and student.

For Strom, contributions to the theater percussion repertoire include those in which the composer extrinsically instructs various extramusical components. However, Mason notes "through detailed study and performance of pieces such as these, a performer can integrate concern for visual and theatrical elements into future performances of theatrical music and also

\footnotetext{
100 Ibid., 17.

101 Ibid., 13.
} 
of absolute music (in this case, with no [prescribed] extra-musical or theatrical elements)."102 This may suggest that integrating extramusical, theatrical performance features into works without those facets instructed on explicitly by the composer "provides the audience with a more complete and encompassing artistic experience," ultimately creating a more individual interpretation. ${ }^{103}$ Therefore, theater percussion perhaps should not be its own subgenre of multiple percussion, as Strom suggests, but rather a dimension of the performance practice that, although stressed in certain works of multiple percussion more than others, should appropriately be applied to all works in the solo multiple percussion medium.

The inclusion of this chapter is meant to suggest that the contemporary multiple percussionist much reach beyond effective fluency of the other dimensions if they seek to master our performance practice. For instructors, the development of efficiency in equipment and setup, notational reading and interpretation, and technical prowess does not constitute a comprehensive program; the curriculum needs to incorporate the development of students' conceptual performance skills. Facets of a conceptual performance are gesture and movement, memorization and internalization, speech and other vocalizations, acting, and staging. Representative pieces in the repertoire were chosen for their explicit instructions on these facets, which an instructor may intuitively use to create learning objectives. Providing students with tangible instructions to build meaningful, practical experiences in the dimension may best prepare them to construct their own conceptual performances for works that do not explicitly prescribe these features.

\footnotetext{
102 Mason, "The Synthesis of Artistic Elements in Works for Theatrical Percussion," 4.

103 Ibid., 1.
} 


\section{Gestures and Movement}

According to Schick, "physicality and gesture in percussion music are powerful tools of communication."104 Movement constitutes both technical and conceptual aspects in the multiple percussion performance practice. Within the technical perspective, movement is viewed as tasks such as efficiency of movement and direct shifting that can be objectively critiqued. The performer should seek to execute these techniques properly, because ignoring them will restrict their overall technical capabilities. However, other movements require the conceptualization of the performer as interpretations of their realization. These, in turn, are subjectively judged by the audience, depending on a complex series of attributes such as their "background, education, experience, lifestyle, and personal taste ...not to mention location in the room, where the person is looking, and even attention levels throughout the performance."105

According to Schick, "the way a percussionist looks and moves on stage is among the most important and highly personal aspects of percussion playing." 106 These are rarely directed by the composer or score: in fact, there may be no direct link between the musical passages, written or aural, and the choreography of gestures and other movements. A rare exception appears in Sen VI (1993), in which composer Toshio Hosokawa details the performer's slow, deliberate and excessively large preparatory motions in the score. ${ }^{107}$ Attention should certainly be paid so that there are not technical or musical consequences for these dramatic movements, Schutz even proposes that "beautiful gestures cannot compensate for incorrect notes or a lack of phrasing... [and] cannot counteract a lack of preparation or improper technique."108

\footnotetext{
104 Schick, 140.

105 Tyson Voigt, "Hearing What You See: A Case for the Use of Ancillary Gesture in Individual Percussion Performance" (DMA document: University of Miami, 2016), 34 - 35.

106 Schick, 141.

${ }^{107}$ Ibid., 147.

${ }^{108}$ Michael Schutz, "The Mind of the Listener: Acoustics, Perception, and the Musical Experience: Do Longer Gestures Make Longer Notes,” Percussive Notes 47, no. 5 (November 2009): 26.
} 
The awareness of one's spatial orientation has direct implications to how movement can add to a musical performance and influence the audience's perception. Petrella suggests striving for fluidity and efficiency of motion between instruments, saying that percussionists "imagine that they are performing within a three-dimensional grid." 109 In my interpretation, each stroke has movement across the horizontal $x$-axis, vertical $y$-axis, and the forward/backward $z$-axis. The z-axis requires specific attention because percussionists may not innately consider what is happening behind them, i.e., what we cannot see as performers but is readily accessible to the audience.

In addition to the execution of a technique, gestures may also play a role in a performer's conceptual performance. What kind of gestures will best service a musical moment? The performer should consider how a gesture will occupy the space and the perception of the audience, i.e., time and space. The perception of one's audience is abundantly conceptual because we never really know their perception. In the preparatory stage, the performer can only imagine how one will perceive the events. In the act, the performer must be sensitive and receptive to real-time feedback from the audience, but cultural taboos limit the audience of Western art music to being passive. The performer therefore must be extremely sensitive if they are to modify their performance based on immediate feedback.

Strom finds that "only a select few forms of musical art...are entirely response-reliant on a live environment - theater percussion being one of them." ${ }^{110}$ Donald Martino's Augenmusic (1972) serves as Strom's exemplar work, which requires live performance for effective visual context. This component is alluded to in the title, as Augen is German for "eyes." Composer notes state that the "female [percussionist] strips her clothing through the piece, an action that

\footnotetext{
${ }^{109}$ Petrella, 24.

${ }^{110}$ Strom, 25.
} 
would undoubtedly provide a connection... between audience reaction and the performer's interaction." ${ }^{" 111}$ While it could be argued that any audio recording stripped away from the visual context lacks affective, intrapersonal, and experiential elements, the physicality of percussion and the structural importance of the performer-audience dialogue in theater percussion, specifically, intensifies the necessity for live performance.

Schutz's empirical research relating to audience perception has led to appropriate hypotheses for the multiple percussion performance practice. One such research study used video and audio samples of different ancillary gestures provided by well-known percussionist Michael Burritt, measuring the resultant length of notes (a) scientifically and (b) the ways in which three groups (non-musicians, musicians, and percussionists) perceived them. Schutz concluded "definitively that gestures are an effective technique for controlling musical note duration."112 However, it was found that longer gestures to do not produce longer sounds in terms of their physical properties, nor do they influence how listeners heard the sounds apart from the visual context. Since most modern multiple percussionist performance is done live, we must consider the perception of the audience. Voigt conducted similar research, but he makes an interesting observation: "stroke types affect the perception of articulation [emphasis added] as much visually as they do aurally. ${ }^{" 113}$ While performance technique can affect perceived articulation, Voigt makes no reference to note duration. Both authors found that the visual component of gestures can effectively mold perceptions in the minds of our audience, which heightens their importance beyond technique and into the realm of drama. According to Strom:

"Even with the natural immediacy of sound decay that percussion instruments possess, a single drum stroke can be perceived short or long solely based on the

\footnotetext{
${ }^{111}$ Ibid.

${ }^{112}$ Schutz, 26.

${ }^{113}$ Voigt, "Hearing What You See," ii.
} 
performer's physical movement, which is achieved in the motions prior to, and immediately upon release of the stroke. The visual component to most non-toned percussion instruments is absolutely necessary for one to appreciate a performer's musical purpose." 114

A gesture may serve to preface a note, subsequently calling attention to the note and activating a specific muscle group, as well as imitate the shape of a musical phrase and influence the perception. In standard notation, a musical phrase is notated by a phrase marking or slur line, a symbol that resembles a simple arc. The plot points of the long gesture can clearly imitate the conventional phrase marking, referencing such an association to the phrase with the performer's fluidity and focused engagement throughout the procedure.

Fluid gestures are an integral concept in William Hibbard's Parsons' Piece (1968), which calls for twelve large, resonant instruments whose "vertical arrangement...means that every instrument is clearly visible to the audience." 115 The work is inherently physical because the percussionist is required to make large motions just to navigate the setup. However, there are longer and grander notes in the piece, so the performer must negotiate when to make grand gestures and when to subdue those instincts to embody a subtler stance. In this regard, Schick warns against exaggerated, extraneous gestures on small instruments, so as not to appear "false" or "pretentious." 116 Charles states that "the gestures created become an integral part in this piece, allowing it to become somewhat of a meditative dance for the performer, tapping into new elements of motion unseen in more compact pieces." 117

Body movement at the highest level would manifest in a work where choreographed dance is primary, perhaps pushing the musical qualities to a secondary role. Vinko Globokar's

\footnotetext{
114 Strom, 23 - 24

115 Schick, 144.

116 Ibid., 147.

${ }^{117}$ Charles, 50.
} 
?Corporel (1985) is a representative work in the multiple percussion repertoire, a piece of much discussion in scholarly discourse and that continues to be performed regularly for recitals. The work is scored for no instruments but the human body, and "in fact, the movement of the body comprises a greater proportion of the piece than the sound component."118 The performer is to be semi-nude and put in various positions such as seated and laying on the ground, all of which are counter to typical Western performance and potentially uncomfortable for performer and audience alike. The performer is instructed to make a series of non-lexical sounds and to hit, scratch, and rub themselves, all of which evoke a primordial character. The piece contains an unclear narrative and only a single quote by René Char toward the end of the piece: "I recently read this remark: The history of mankind is a long succession of synonyms for the same word. It is a duty to disprove this." ${ }^{\prime 19}$ In this way, the performer must contemplate the little spoken poetry and very minimal background information provided by the composer to determine the conception and narrative, if any, of this highly choreographed and theatrical work.

\section{Memorization}

Memorization and internalization should be integrated as a definitive part of the percussion curriculum. There are applications in various professional contexts, such as jazz, popular, world music and free improvisation, where reading sheet music on a music stand would be uncharacteristic of the style. Of course, memorization can also add to one's presentation of select advanced solo multiple percussion literature. Techniques that describe how to memorize efficiently go far beyond the scope of this document, but interested readers should refer to Schick, who describes his process of "chunking" and other tools for effective memorization. ${ }^{120}$

\footnotetext{
118 Strom, 80.

${ }^{119}$ Vinko Globokar, ?Corporel (New York City: C.F. Peters Corp., 1989), score.

${ }^{120}$ Schick, 126 - 127.
} 
The focus of this section is the concept, when, and why one might seek to engage in memorization/internalization and incorporate it into the percussion curricula.

Steven Schick has stated that he memorizes all solo works, but he recognizes other perspectives and avoids a pretentious stance on the subject:

"For most performers of contemporary music who choose not to memorize, playing from score is a philosophical and not a practical decision. They do not avoid memorizing to save time or out of the fear of forgetting. At best their performances are as profound and deeply felt, as grounded in reflection, intuition, and indeed muscular memory as an Horowitz interpretation of Rachmaninov. No, theirs is a decision to store musical information on the stable medium of paper rather than in the human body..."121

In fact, there are various detrimental aspects of memorization. Schick warns his readers of the "affliction of memory," the basic natural human tendency to warp memorized music over time. He also argues that memorization leads to a degree of ownership, which comes with freedoms that are both advantageous and disadvantageous. I must admit having felt a certain level of authority when the sheet music became unnecessary to perform a work, but the tendency for the brain to mold information puts the percussionist at odds.

There are many advantages to bypassing memorization in favor of reading the score during performance. Using sheet music will counteract mistakes and the warping of music information in the learning process, as well as rendering the fear of forgetting in performance virtually powerless. Audiences may consider score reading as external proof that the "player is not simply inventing on the spot," thereby validating the performance as credible." ${ }^{122}$ The percussion instructor should seek to establish a culture in the studio where memorization is not viewed as "better" or "more impressive," nor an additional "extra credit" task a student should

\footnotetext{
${ }^{121}$ Ibid., 121.

${ }^{122}$ Ibid., 120 - 121.
} 
take after a piece has been learned. Rather, memorization should be a conscious decision made for valid reasons.

There are many possible scenarios in which memorizing should be a real objective. Strom posits that the ability to dedicate one's sight for increased "spatial orientation and accuracy" as a prime factor for memorizing a work. ${ }^{123}$ In comparison to other musicians, the fact that we do not typically touch our instruments provides further validity for memorizing, thereby freeing our eye sight. Memorization may be inappropriate for instrumentations with imprecise or large playing spots, such as the large cymbals and tam-tams in Parson's Piece. Although select passages would benefit from memorization, it should not be a point of major emphasis for Thirteen Drums. Instead, the drums closest to the music stand should be located within the player's peripheral vision while looking at the score, while the drums closest to the player should be located primarily though muscle memory.

In terms of the conceptual performance, the percussionist should ask themselves if and how memorization can add to the performance. Conversely, how can memorization actually detract? These questions should be asked as early as repertoire selection, which would allow memorization to be applied to the learning process, à la Steven Schick’s process. The visual freedom that Strom posits contains increased dramatic options as well as technical accuracy. One could look directly at an instrument to transmit the importance of a given note or moment, for example. Coupled with facial expression, closing the eyes during a passage aids greatly in presenting a moment of reflection or even fear. The dramatics can be applied to memorized passages but would be even more effective if executed in a piece with the full score memorized

\footnotetext{
${ }^{123}$ Strom, 50.
} 
because their nuance would be more perceivable if the physical music stand and sheet music were not obscuring the view of the audience.

For those that may have not played a piece from memory, an appropriate choice is one that is built on structures and process. In this way, the student must only keep the structural components in mind and is not preoccupied with playing right or wrong notes at a micro-level. With this curriculum in place, fear is minimalized in favor of expression and virtuosity, and the student builds their confidence in memorization. Examples of this type of process structure are Pauline Oliveros's The Single Stroke Roll Meditation, John Cage's Child of Tree, and James Tenney's Having Never Written a Note for Percussion.

Students that have some experience in memorization should be guided to solos that are built on repetitive patterns and variations. David Mancini's Latin Journey offers multiple occasions where interesting changes are relegated to one hand, which occurs over an established ostinato in the other. The very opening of the work contains a two-measure ostinato in the woodblock, which is repeated for the first sixteen measures (Fig. 5.1a). After two such iterations of the woodblock, the player's right hand enters with cowbell half notes for the next four bars. For the last eight measures of the section, the cowbell changes from half notes to a typical cascara pattern. Although notes do not change, the musical crescendo should be used to help distinguish the last four measures of the section, such a structure point also aiding in the memorization process. The next section utilizes four-bar phrases as well, built upon easily recognizable call and response (Fig. 5.1b). The two-bar call is repeated at the beginning of each four-bar phrase, which diminishes the amount of material that the player must learn and recall in performance. Although each response is different, the fourth response (measure 32) is simply the second response (measure 23) with rests in lieu of the material in the first two beats of the second 
measure. The regular phrase construction, repetitive patterns, isolated patterns for each hand, and definitive dynamic changes serve as structural points that can aid in the player's memorization of the material.

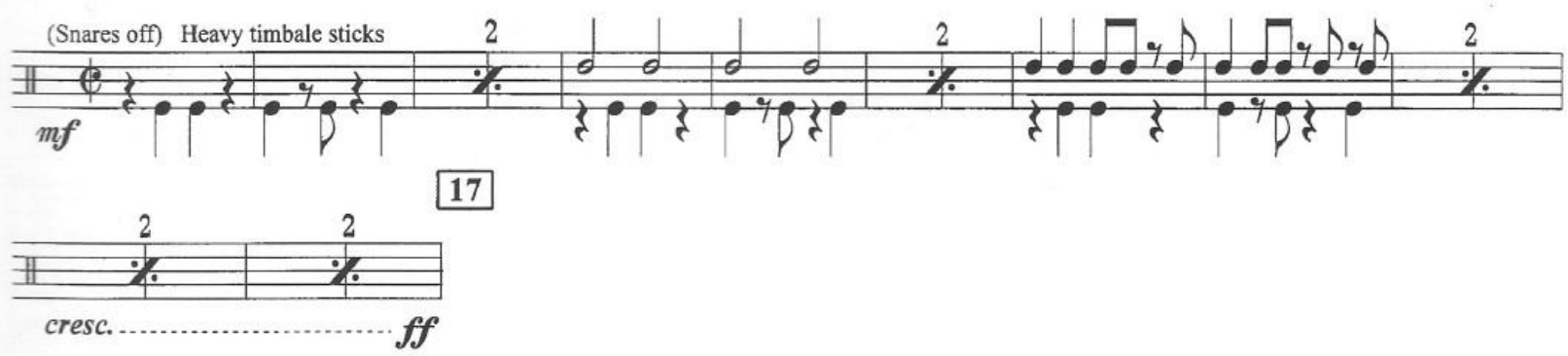

Fig. 5.1a Latin Journey, left hand ostinato

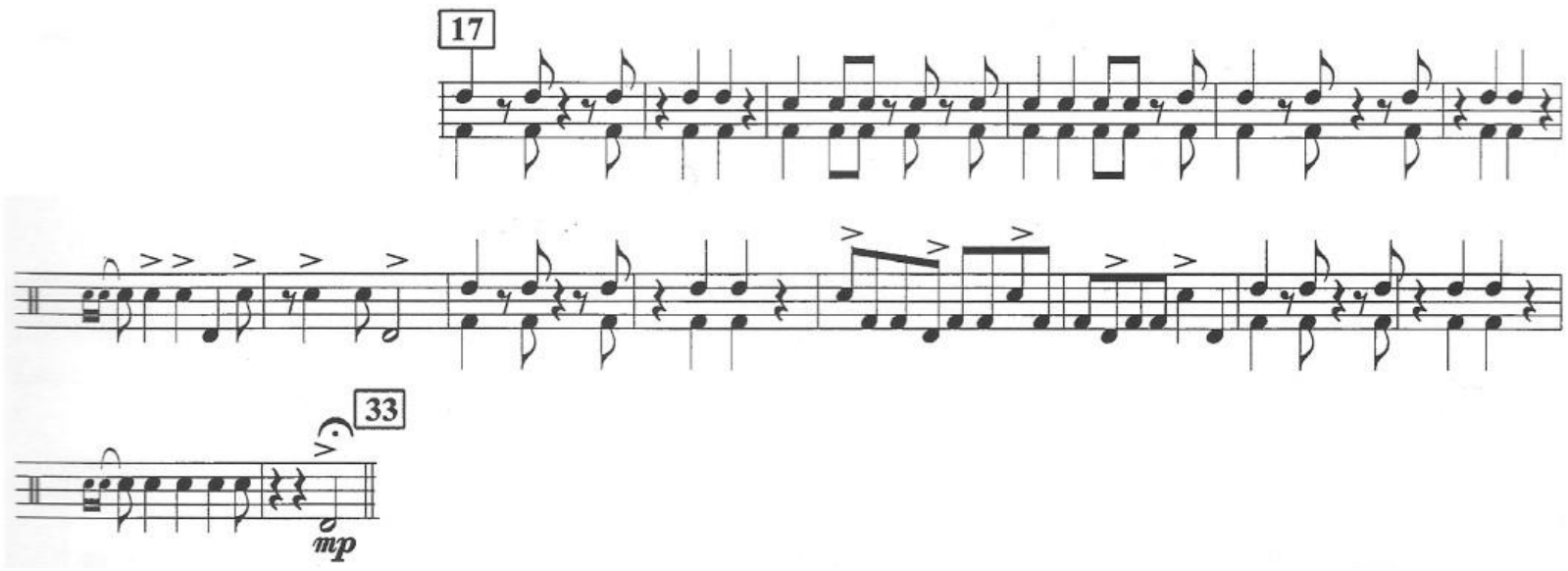

Fig. 5.1b Latin Journey, call and response

Despite its repetition, Michio Kitazume's Side by Side presents an added difficulty to memorization. The piece contains modules that are to be repeated a various number of times (Fig. 5.2), ultimately diminishing the amount of material in the work. In addition, the player may choose to rely on muscle memory to continue a pattern after it has been established, thereby allowing themselves to think ahead even audiating the next material before moving to it in their performance. These modules range from the shortest of a single note to the longest, lasting dozens of notes. Plus, repetitions are directed to last from a single repetition to forty-six, making 
for an uneven memorization task. In addition to some modules being indicated with a range of repetitions (ex: 7 - 9), Kitazume suggests these to be viewed as guidelines, allowing the performer the freedom to change repetitions to "create his or her own sense of rhythm." The performer can create repetitions that are more easily remembered, based on numerical patterns or other numbers that are meaningful to them. The freedom to alter repetitions of the material may also create a failsafe in a performance situation: if a memory slip occurs, the player can simply continue the ostinato while they regain their composure and place in the music. Although the piece requires the performer to play constantly for its approximately ten-minute duration, the repetition and the player's freedom creates an easier task than works that are more throughcomposed.

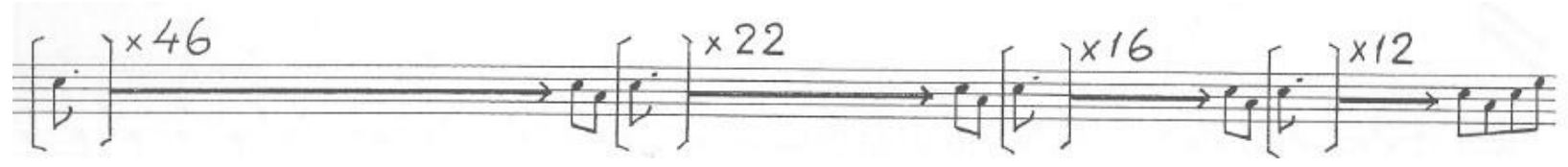

Fig. 5.2 Side by Side, opening modules

The level of difficulty in the memorization task and the capabilities to incorporate desired theatrics should influence one's decision to memorize. However, factors of staging, such as the layout of the venue, should also impact memorization and other conceptual components.

\section{Staging}

The live nature of the percussive art form brings elements of staging to the forefront of a multiple percussion performance. The venue of the performance will influence other choices that should be made. Certain programs may require students to perform degree recitals in a designated venue: a formal recital hall. However, students and instructors might consider other creative options, even seeking an exemption to program requirements when applicable. The theater and dance department may have a venue with a thrust or arena stage, or a black box 
theater. Performances in these types of venues will create logistical problems and performance problems that the student should creatively solve with the guidance and mentorship of the instructor. The education system should strive to prepare students for performing in different spaces and different kinds of venues, considering that is what the vast majority experience as professional musicians. Gaining firsthand experience in promotion, logistics, and performance in an outside venue will help prepare students in the professional realm.

Stage placement and logistics are obligatory skills for the percussionist. The conceptual and creative process and dramatic performance can intertwine with the equipment and setup dimension. These are often solely the percussionist's responsibility in the solo realm, Acoustics will influence a setup's position up or down the stage, but attention should be paid to the sightlines and the rake (grade of the seats) of the theater's house as well. Placing a setup stage right or stage left may or may not affect overall acoustics, depending on the construction of the venue and the piece's setup design. Larger multiple percussion setups that wrap around the performer will demand special attention because certain instruments will inherently be placed closer to the audience than others. Consider placing larger instruments upstage and smaller instruments downstage so that the audience will have sightlines for all instruments. Be aware that instruments that are closer may resonate longer and sound louder from the audience's perspective. It is advised to have colleagues or stagehands check for these various components, sound checking from different vantage points from the house. In general, recording yourself from a single device will not suffice in getting an accurate picture of the audience's experience.

Costumes and props mark another staging component for theatricality and drama with multiple percussion performance. These visual elements can aid the auditory and provide a medium in which the percussionist can make a performance unique and relay their individual 
interpretation of a work. Costumes and props may help create a narrative or a story, "introduc[ing] a certain specificity to an otherwise metaphorical performance - a more detailed impression of who, what, when, and where." ${ }^{124}$ Strom describes John Cage's Living Room Music (1940) as a monumental use of theatrics with particular attention to costumes and props. Cage directs the players to use "household objects or architectural elements" rather than conventional instruments that are not to be struck with conventional beaters. The dramatic acting "is a result of the environment instead of a product of dramatic/choreographic directions expressed by Cage." ${ }^{125}$ Performers often choose to stretch the unconventional nature of the work by leaving formal concert attire behind, using pajamas or business attire to portray 1940s' homelife. As the props are also the musical instruments in the work, their visual properties should be considered equally with their sonic properties (see Chapter 2). The management of the size, shape, and color of each prop adds to the individualism. The audience must be pulled to focus their attention on the desired props at any given moment, so distractions from the other musicians must be limited, downstaging for clear sight lines, and the blocking for each prop and musician on stage should be considered. For example, the massive size of the newspaper can greatly add or detract from the theatrics of Living Room Music. It can be positioned to display a particular section or headline that can be seen by the audience, thereby associating the performance with a particular time and place, suggesting a sociopolitical stance, and even portraying the character on stage. Although Cage does not prescribe its directions, the prop instruments, costumes and the oral tradition of this commonly performed work have created standard theatrical practices in performances of Living Room Music.

\footnotetext{
${ }^{124}$ Strom, 28.

${ }^{125}$ Ibid., 72.
} 
In the solo realm, Stuart Saunders Smith ...And Points North (1990) significantly uses staging to convey theatrical drama and the conception of the piece. Smith indicates that "all the elements of performance - movement, sound, text, theater - should all be consciously considered." Some even consider the work a "percussion opera in three movements" due to its holistic integration of theatrical elements. ${ }^{126}$ In the performance notes, Smith specifically suggests the performer's facial gestures, stage logistics and setup, lighting, and costumes. Additional notes are provided by Steven Schick, who further suggests changes throughout the piece in terms of character theatrics and gestural treatment, such as mechanical versus fluid movement.

Stuart Saunders Smith provides the performer with the inspiration and conception of the narrative that is built on three scenes, each of which are to be performed in its own space on stage. Wadley suggests that sets "take advantage of the full depth of the stage" to add dramatic effect and simulate the passage to various cultural concepts of "north." 127 The performer must recite poetry in both English and Native American Passamaquoddy. Smith provides the phonetic instructions to aid in pronunciation, but the meanings of its translation must be internalized if the theatrical expression is to be conveyed to the audience. Two trees are the center of the second movement setup, which the performer must sensitively play while the acting, movement, and recitation can all be viewed by the audience (Fig. 5.3).

\footnotetext{
${ }^{126}$ Lee Hinkle, “Theatrical Music for Solo Percussion” (DMA document: University of Maryland, College Park, 2010), 7.

${ }^{127}$ Darin Wadley, “...And Points North by Stuart Saunders Smith: An Examination of Musical Influences and a Performer's Guide” (DMA document: University of Arizona, 1998), 105.
} 


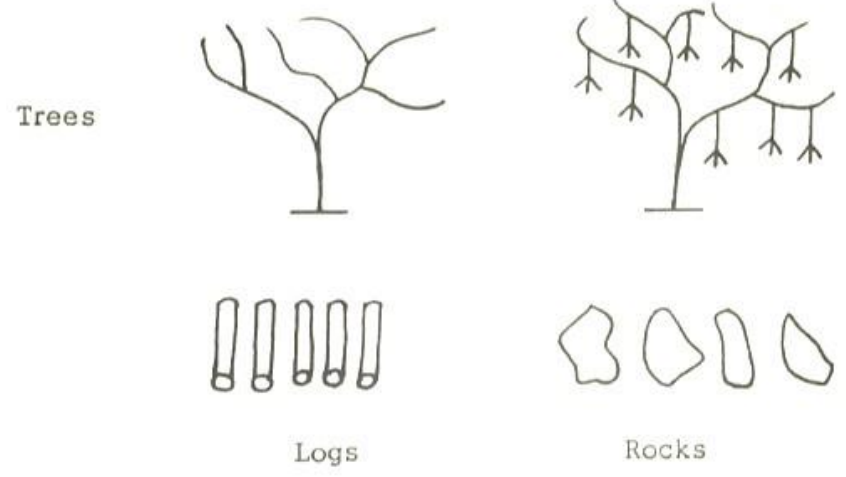

Fig. 5.3 ...And Points North, Movt. II, setup diagram

Lighting is another aspect of staging that demands specific attention in select pieces of the repertoire. Smith provides a simple direction for lighting in ...And Points North: "Lighting should be designed to create shadows and pools of light to bring out the visual poetry of the set." ${ }^{" 128}$ In order to create shadows and highlight the specific scene of the narrative's continuum, specific setups should be illuminated, while other portions of the stage are dark. Wadley suggests lighting the final gestures of the piece with only the "silhouetted shadow of the percussionist visible" and that ending with a slow fade would help portray the character as "he/she transcends the physical world and enters the spiritual world."129

Casey Cangelosi provides explicit lighting directions for Theatric No. 7 (2010), in which a metal lamp provides the only light for the performance. In the work, Cangelosi gives precise timings for the performer to click an on/off foot switch directly in the musical score. In this way, the lighting is completely choreographed and the full responsibility of the performer in real time. Since the percussion music continues even in moments when the lamp is turned off, memorization of select passages is required in the work.

\footnotetext{
${ }^{128}$ Stuart Saunders Smith, ...And Points North (Sharon, VT: Smith Publications, 1990), music score.

${ }^{129}$ Wadley, 107.
} 
Dorothy Hindman's Tapping the Furnace (2006) is an advanced theatrical piece for speaking percussionist. The piece contains many technical challenges, such as a fast 7/16-time signature, specific playing spots, and an array of extended techniques. The coordination between percussion and the spoken text presents difficulties in timing and balance. There are large spaces between the spoken text during which dense percussive counterpoint occurs, posing a challenge for the percussionist to imitate natural vocal inflection and cadence. Exaggerated movements and gestures are not possible during sections of great technical demand on the snare drum and toms, which makes the theatrics during the sections where it is possible, such as the coin bucket passage and the long, open spaces, even more significant. Charles describes lighting directions:

"Hindman gives explicit instructions for lighting the stage to create a more dramatic performance, shifting the stage lighting from a deep red through orange and yellow, arriving at white and then fading out to black with a spotlight on the performer." ${ }^{30}$

In the case of Tapping the Furnace, the lighting will have to be predetermined, precisely timed out to be controlled by an external operator or programed into a computer. While staging processes such as logistics, costumes, props, and lighting typically occur prior to a performance, elements such as dramatic acting require practice for fruitful execution of one's concept in real time.

\section{Dramatic Acting}

The application of extramusical acting can consist of reevaluating one's movement, incorporating facial expressions, or any other technique to create dramatic effect. Given the experiences in the aural aspect of absolute music provided by contemporary education systems, percussionists may be apprehensive about applying dramatic acting to a solo performance. Truly exposed portions of solo percussion music surface from long notes, rests, and silences, in which

\footnotetext{
${ }^{130}$ Charles, 40.
} 
case a performance engulfed solely in absolute music becomes meaningless to the live audience. These exposed moments can be utilized to introduce ways in which dramatic acting can add to musical interpretation and performance.

Tomas Svoboda's Discernment of Time (1983/2003) can serve as an introduction to the concept of reevaluating movement. The percussionist is presented with a number of advanced techniques that are notated with a unique notational system, both of which require precise assessment at the onset of learning the piece. The student should pay close attention to detail to create a meaningful, distinct vocabulary that is unique to this specific piece. Precise playing spots on the gong and the gong stand, as well as the specificity of implement, including the top and middle of a chop stick, is necessary to bring the piece to fruition and communicate the extramusical associations to the audience. The composer does not give explicit directions on performance aspects, such as where the percussionist should position themselves: to stand or to sit, and whether that is in front or behind the gong, presumably leaning over the gong to play on the traditional playing surface in the latter. Charles observes additional theatrical opportunities "as the surface of the tam-tam is used to represent a clock face."131 The conclusion is supported by the title of the work, the metronome marking being $60 \mathrm{bpm}$, and the division of the gong into twelve equal units along the edge (Fig. 5.4).

${ }^{131}$ Ibid., 32. 

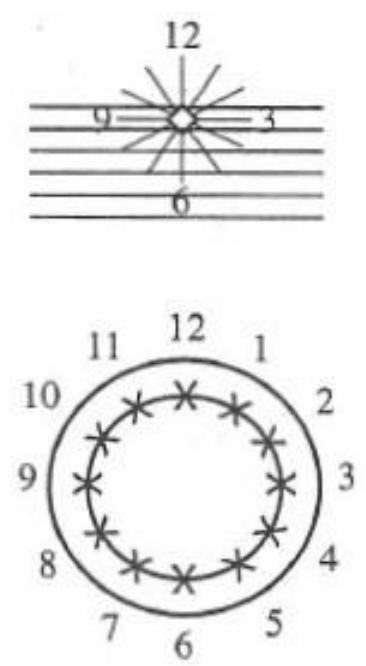

Fig. 5.4 The Discernment of Time, clock face association

In my opinion, the percussionist can imitate the hands of a clock by repurposing their movement with angular, staccato strokes. Furthermore, the player's elbow should be located at the center of the clock as much as possible. To maximize these theatrical moments, the percussionist will have to choreograph movement around the instrument throughout the piece because these theatrical nuances are moot unless the "clock face" can be viewed by the audience.

George Lewis presents similar problems in dramatic acting in his solo with pre-recorded tape, North Star Boogaloo (1996). However, there are virtually no absolute silences in the work. While the percussionist rests, the pre-recorded tape sounds at full force, including hip hop beats, readings of the Quincy Troup poem of the same name, and interview quotes from basketball players Michael Jordan and Charles Barkley.

"A bigger problem yet was how to look and act during pauses in the playing while the taped hip-hop music went on alone. How should I act when there are no tasks?"132

\footnotetext{
${ }^{132}$ Schick, 74.
} 
A performance lasts over fifteen minutes, which explores connections between the north star and basketball as avenues toward socioeconomic freedom and prosperity in America. Steve Schick discusses his insecurities in performing this piece that utilized the materials of the urban African-American community, which challenged his preconceived thoughts on being the “expert...[as] a white middle-aged, classically trained percussionist intently focused on accurately rendering hip hop rhythms to a mostly white, mostly middle-aged audience."133 The performer must decide how they will act when not physically engaged in making sound, as well as the social implications of those actions.

Facial expressions are an integral part of the drama in any musical performance. Unless an extended technique requires something different, the percussionist has full facility over their facial expressions in percussion performance. It is important to remember that while the attention of fellow percussionists and musicians may gravitate toward the technique and instruments, nonmusicians are likely to be watching the performer themselves. Stuart Saunders Smith's Songs I IX (1981) is composed for an actor-percussionist playing mostly found objects and includes spoken text in both English and gibberish. In addition to the theatrics of the props and vocals, Songs $I$ - IX is an advanced conceptual piece due to the "rapidly changing demeanor throughout the entire range of human emotions." 134 Since the performer vocalizes throughout the work, the attention of the audience is often drawn to his/her face.

Smith also asks the performer to develop different characters over an extended time in Songs I - IX. Their narrative and characterization through mannerisms are brought to life with detailed research and analysis of typical behavior. Since the performer must quickly juxtapose

\footnotetext{
${ }^{133}$ Ibid.

${ }^{134}$ Charles, 39.
} 
between characters, they must have full command to clearly portray the different roles to the audience. Particular attention should be taken for the "Song V" character:

"Deliver as a faith-healer, Bible-wheeling very southern preacher. Use many word tremolos for emphasis... Your gestures should look like this type of preacher. The mallet-table and maraca should look like part of the religious speech." 135

Smith does well to keep his instructions descriptive, allowing the performer to construct an interpretation that could range from honorary tribute to satirical insult. The "faith-healer" character may bring the performer's prior religious ideas, associations and even biases to the surface. In addition to self-reflection, measurement of the expected audience and the regional culture of the audience should influence the degree of exaggeration needed for the performer's portrayal to be perceived as intended.

\section{Vocals}

Spoken text, singing, and other vocal sounds are utilized in solo multiple percussion works of various styles and instrumentations. Vocal style is an important aspect to execute a timbre that is fitting to the music. When using one's voice, attention should be paid to diction, as proper enunciation will help with the clarity within a specific vocal style. Utilizing an accent may further display virtuosic skills and establish a setting or scene. Composers typically realize that classical singing is not the percussionist's forte, so guidance sought should be within spoken diction rather than classical singing diction. Nevertheless, guidance from faculty in the voice and theater departments is recommended. Recording oneself and listening back is a useful tool for determining if the volume, timbre, pitch, and diction are appropriate, in general, and true to the performer's concept.

\footnotetext{
${ }^{135}$ Stuart Saunders Smith, Songs I - IX (Sharon, VT: Smith Publications, 1981), music score.
} 
Much like diction, a working knowledge of poetry is vital to understanding the treatment of text. A useful resource for understanding poetry in its spoken form is The Sounds of Poetry by Robert Pinsky. Pinsky articulates how poetic feeling and meaning is created via the synthetic treatment of separate elements: accent and duration, syntax and line, like and unlike sounds, and formal considerations such as blank verse and free verse. ${ }^{136}$ Like music, Pinsky's aural stance on the art form should resonate well with musicians, as he states that "the [poetic] line is vocal, a sound; the typographical arrangement is a notation for that sound." ${ }^{137}$ Regardless of expertise and confidence, instructors should encourage percussionists to add pieces with spoken language to their repertoire, despite the solo multiple percussion artform already being quite complex without that added element. A well-formed curriculum that spans levels of difficulty in the spoken realm will help realize this skill in student percussionists.

Frederic Rzewski's To the Earth is a great introductory piece within the conceptual dimension that specifically focuses on vocals. Besides finding four flowerpots and the appropriate implement to strike them, the equipment and setup, notation, and technical dimensions should present little trouble for the intermediate percussionist. A difficult task lies in the clarity of the spoken vocals in general, but also that the percussionist must follow Rzewski's directions that the "words are spoken more or less together with the music." The vague language here suggests a level of harmony between the voice and instrumental accompaniment, but perhaps also hints that they are also two separate, independent lines. While the two lines sound in unison during the first section of the piece, they drift farther and farther apart as it progresses. The finale operates more like a call-and-response, with the voices never sounding

\footnotetext{
${ }^{136}$ Paraphrase of Robert Pinksy, The Sounds of Poetry: A Brief Guide (New York: Farrar, Straus and Giroux, 1998), 97.

${ }^{137}$ Pinksy, The Sounds of Poetry, 109.
} 
simultaneously. Regardless of the homorhythmic or counterpoint texture, preserving proper speaking cadence may prove difficult, especially for those unfamiliar with spoken poetry, monologues, and other theatrical dialogue. Despite the rhythmic appearance of the score, an overly measured, robotic recitation would be counter to the poem's earthly theme.

Considering the worldwide attraction to Western multiple percussion solo performance, it should be no surprise that multiple percussion solo texts occur in a variety of languages. The use of the Native American Passamaquoddy language in ...And Points North was described previously, for example. Vinko Globokar's Toucher presents advanced challenges for the percussionist, who is directed to speak scenes from Bertolt Brecht's play Life of Gallileo. The percussionist speaks these scenes in French, while performing a chosen sound to match each of the seven vowel sounds heard in the play. The percussionist must orchestrate sounds that imitate the phonemes, "linking" the sound with its verbal association. This can present significant problems for those unfamiliar with French and unaware of the subtleties of its pronunciation, especially when orchestrating musical sounds that are meant to imitate vocal ones. Yet, if careful attention is paid to diction and instrument choice in the early steps of one's realization process, then the two elements will reinforce each other and ultimately make for an accessible experience with a manageable learning curve for even an undergraduate student.

Other works avoid meaning within spoken language by incorporating non-lexical sounds. Georges Aperghis uses such techniques in much of his repertoire, including the trio Les Guetteurs des Sons (1981), where the "performers explore phonetic sounds, creating an analogy between vowels and consonants." ${ }^{138}$ Since sections contain spoken French text that maintains proper syntax, the performers must negotiate how the vocal extremes intersect to create meaning

\footnotetext{
${ }^{138}$ Strom, 27.
} 
in the work. It also contains explicit directions for mimicked "air" playing, facial expressions, and other theatrical elements that display that "the hands of the percussionists sometimes work independently of their bodies. They themselves are surprised..." ${ }^{139}$ Les Guetteurs des Sons is a monumental piece for the percussionist, as is Stuart Saunders Smith's Tunnels, which also explores the interplay between syntactic language and non-lexical vocals, presenting a myriad of advanced problems that require conceptual fortitude from the solo multiple percussionist.

\section{Stuart Saunders Smith, Tunnels (1982-85)}

Performed on April 25, 2020, Final DMA Recital in College Hall, West Liberty University.

Stuart Saunders Smith composed Tunnels between 1982 and 1985. The piece was published in 1988. Smith continues to be one of the most intriguing compositional figures in the modern era, drawing scholarly attention from researcher-writers and performances by student and professional musicians. An intriguing aspect of the composer is his varied style, which proves difficult to generalize. Yet, those familiar with the composer arguably know his works quite instinctively and the style as being distinctively Smith's own. A complete overview of the composer, his style, and works is inappropriate in this document, but interested readers should consult John P. Welsh's The Music of Stuart Saunders Smith for a comprehensive analysis.

Welsh concludes his book with a study of Smith's poetic devices, particularly how they serve as formal markers in Tunnels. Speaking of the multitude of theatrical and percussive elements, Welsh states:

"Smith enlarged his notion of speech songs in recent works to include staging...elevated to the level of mini-opera in Tunnels, Songs $I$ - IX and ...And Points North where acting, props, and stage sets as well as singing, speaking, movement and playing on instruments are required." 140

\footnotetext{
${ }^{139}$ Georges Aperghis, Composer Website: Program Notes: Les Guetteurs des Sons (accessed February 16, 2020). http://www.aperghis.com/notices.html

${ }^{140}$ John P. Welsh, The Music of Stuart Saunders Smith (Westport, CT: Greenwood Press, 1995), 152.
} 
The scholarly literature on Smith's theater percussion style, including those studies that analyze his three "mini-operas," continues to expand, even while he is still composing new music. Both Welsh and Sofin provide historical and cultural context that builds a requisite foundation to understand Smith's compositional style, emerging via comparison between other representative movements and individual composer-practitioners in twentieth-century theater-percussion. ${ }^{141}$

Sofin focuses specifically on Smith's works for spoken text and instrumental music, providing a comprehensive synthesis in which Tunnels can best be placed. ${ }^{142}$ While Welsh maintains the focus on poetic analysis, Sofin sympathizes with the multiple percussionist with a focus on performance practice.

For pedagogical purposes, Tunnels demonstrates an exemplar piece in the conceptual performance dimension because it exhibits requisite extramusical elements for the modern percussionist and can serve as a valuable resource in instructors' curricula. Smith's other theaterpercussion works that integrate voice and instrumental accompaniment could also serve the student and instructor well, which can be found in the curriculum appendixes. The deciphering, learning, analysis, realization, and performance phases of Tunnels, specifically, are a true conceptual feat. Sofin describes the advanced role the performer serves as part-composer:

"Instead of assigning the instrumental sounds to appear with the same syllables every time, they appear in interplay with Smith's words. This system allows for more freedom, because unlike the pieces by Aperghis and Senn, the rhythms of the hands and text are not tied together."143

The compositional responsibility of the performer is just one element that makes Tunnels an exemplary work in the conceptual dimension. One conceptual element that surfaces

\footnotetext{
${ }^{141}$ Ibid. $54-55$.

${ }^{142}$ Elizabeth Sofin, “Text as Music, Music as Text: Stuart Saunders Smith's Works for Percussion and Spoken Word," (DMA document: University of Arizona, 2017).

${ }^{143}$ Ibid., 59.
} 
throughout the realization process is recognizing which elements are explicitly directed on by the composer and which are directed implicitly; similarly, which elements are completely void of the composer's influence and thereby entirely the responsibility of the performer.

An interesting facet of Tunnels is that the technical dimension is not prescribed by the composer. In this way, the percussionist controls the level of technical difficulty and can cater the technical demands to suit them. Similarly, the percussionist has a great deal of control in the equipment and setup dimension as well. Smart instrument choices need to be made to offer a variety of sounds (pitch, duration, articulation, release, dynamic), but the level of extravagance and complexity in their setup design is entirely in the percussionist's purview. While there is potential for minimizing the technical and equipment/setup dimension challenges, the advanced notational demands in Tunnels will require the percussionist's attention.

The score for Tunnels resembles a poetic script, rather than a traditional music score. Three pages of prose "performance directions" communicate an enormous amount of information for realization. On the other hand, the score itself is less than eight pages, making for an unusual ratio of performance notes to musical score for the student percussionist. Since much of the text consists of single syllables, isolated consonant sounds, and even non-lexical sounds, even the poem itself requires careful analysis. Furthermore, the poem is laced with a variety of non-standard symbols that only add to the difficulty of reading the text.

The graphic symbols are positioned on the page in relation to the text. Three graphic symbols are attached to individual phonemes in the poem, each providing specific performance instructions regarding their treatment. The phonemes are (a) circled, (b) boxed, or (c) circled and boxed. Combinations of the symbols also demand specific treatment, making the symbol vocabulary very complex. Recognizable English words may also be accompanied by graphic 
symbols, such as brackets or zig-zags. There is one instance where the phoneme and word symbols mix (Lines 102 - 07), where typical English words, forming atypical grammar, are boxed. Significant durations are signified by isolated periods that occupy an entire line of text, one period equaling two seconds.

Two graphic symbols are not directly affixed to the recitation of the poetry: the zig-zag underscores and the grace notes. For underscored words, the performer should firstly decide whether to imitate "some aspect of the sound" or whether to interpret the word as a performance direction. Whichever orchestration is chosen, Smith indicates that the musical accompaniment should sound until the next musical notation appears and that the playing

"need not always be continuous sound. The performer can and should add small musical rests to give textured variety and rhythmic interaction with the text. Zigzag passages should be contrapuntal in nature." 144

Therefore, the underscored words clearly contain a great deal of flexibility in terms of both instrumentation and rhythm. Due to their lack of attachment directly to a word or phoneme, the orchestration of grace notes also rests on the performer. Smith indicates that the grace notes represent "instrumental noises" that are not traditionally associated with percussion, specifically referencing radios or found objects. In order to validate this unique symbol, especially since the composer does not reference "noises" anywhere else in the performance instructions, one could assume that Smith is looking for sounds that are not orchestrated elsewhere in the piece (boxes, circles, zig-zag lines). It should be noted that these grace notes never encompass a phoneme, although there are phonemes that imitate the grace notes' slash through the note stem and flag. In Fig. 5.5, a grace note precedes the phoneme "net," while the penultimate phoneme "S" is not a grace note.

${ }^{144}$ Stuart Saunders Smith, Tunnels (Sharon, VT: Smith Publications, 1988), performance directions. 


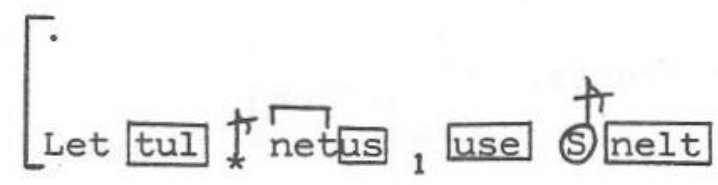

Fig. 5.5 Tunnels, lines 22 - 23, grace note symbol identification

The composer further articulates that five different instrumental noises should be "divided approximately evenly among the seventeen indications throughout the composition." Executing this task proves to be quite difficult, considering thirteen grace notes appear in the A section (lines 1 - 38), none in the B section (lines 39 - 176), and 4 in the A' section (lines 185 212). In addition to the expected instructions concerning the parameters of the graphic symbols used in the score, the performance directions also detail elements of timing, acting/narration/vocalization, and pronunciation.

Smith provides instructions for the performer's learning process, articulating that an initial step in developing their realization is to "thoroughly memorize and develop a vocal interpretation of the text." A significant challenge is posed in the A and A' sections, where the vocables derive from letters, letter combinations, and permutations of the word "tunnels." Without recognized syntax or overt meanings in these sections, I found making a bank of all the phonemes to be quite helpful (Fig. 5.6).

\begin{tabular}{|c|c|c|c|c|c|}
\hline$\underline{E}$ & $\underline{\underline{L}}$ & $\underline{\mathbf{N}}$ & $\underline{\mathbf{S}}$ & $\underline{\mathbf{I}}$ & $\underline{\mathbf{U}}$ \\
el & len & nel & set & te & un \\
els & let & nelt & sten & tel & us \\
elts & lu & nust & stu & ten & use \\
en & lunes & & su & tu & ust \\
et & lute & & sul & tul & ut \\
& lut & & & tun & \\
& luts & & & tus & \\
\hline
\end{tabular}

Fig. 5.6 Tunnels, bank of phonemes 
In addition, two phonemes are occasionally combined in Tunnels. On page one, Smith provides a list of combinations to be pronounced as one word. Some of these "words" are implicitly linked by being encapsulated entirely within one box or circle. However, "netus" on line 23 is one example of a "word" where the partial box instructs musical orchestration for the second phoneme only, "us." I formed a bank of these phoneme combinations as well (Fig. 5.7) to streamline my practice sessions and focus on fluidity. Another strategy may be to annotate the non-lexical vocabulary in the piece with IPA (the International Phonetic Alphabet), a shorthand system for representing sounds from any language that uses the Latin alphabet. However, I bypassed using IPA due lack of experience and practice.

\begin{tabular}{|c|c|c|c|c|c|}
\hline$\underline{\mathbf{E}}$ & $\underline{\mathbf{L}}$ & $\underline{\mathbf{N}}$ & $\underline{\mathbf{S}}$ & $\underline{\mathbf{I}}$ & $\underline{\mathbf{U}}$ \\
eltun & lenus & netus & seton & tenl & untel \\
ensu & luet & nulet & sluent & tutul & \\
entu & & & sunet & & \\
& & & sutle & & \\
\hline
\end{tabular}

Fig. 5.7 Tunnels, bank of combinatory phonemes

In addition to the examples that Smith provides, other combinatory phonemes can be made, depending on the performer's individual interpretation of the score. As described via "netus" in the previous example, the musical notation can influence one's interpretation. The box visually suggests a slight vocal pause in the recitation, but Smith explicitly suggests a fluid recitation is needed. In other places in the score, an interpretation issue arises from the spacing on the page, such as "let tul" earlier in line 23 (Fig. 5.5). The release of the letter " $t$ " at the end of a phoneme suggests a quick rhythm into the next phoneme is certainly plausible, linking together both phonemes into a single word. However, the space between the words in the latter, not visually set in the former example, suggests Smith conceived a different execution. While not 
required for execution of the recitation of the poem, some understanding of articulatory phonetics through resources like Catford would be helpful, especially in developing an informed interpretation of the non-lexical poetry in the A and A' sections. ${ }^{145}$

The primary objective of creating and referencing these two term banks was to learn the new vocabulary, better positioning them for analysis, comparison and practice. Without being able to recognize and execute each phoneme in this isolated fashion, it would be safe to assume that execution with a much more complex, detailed, and dense performance score would be impossible. The analysis of each phoneme commenced with Smith's explicit directions for how each should sound. Despite Smith's suggestion to "memorize thoroughly," I never completely memorized the piece. Instead, I memorized only portions where I thought that looking at the physical score would detract from the theatrics. For example, the absence of text, if not sound entirely, instructed with the period symbol may rouse the audience to devote more attention to the visual information on stage. In these cases, overt reading of the score will ruin the constructed theatrics, the audience even perceiving the performer as "searching" the score for their next material.

After developing fluency with pronunciation of individual phonemes, I began focusing on the details that Smith does not prescribe to formulate ideas about how each could sound. I found it most useful to embark on this exploration in context, firstly line by line. While Smith explicitly guides the pronunciation in terms of consonant and vowel distinction, the performer is left to explore vocal facets such as timbre, articulation, and pitch/pitch movement. This stage of phoneme analysis was a strategy to consciously avoid assuming that my natural, intuitive pronunciation was best. I am neither a professionally trained speaker or poet, after all. For the

145 J. C. Catford, A Practical Introduction to Phonetics, $2^{\text {nd }}$ ed. (New York City: Oxford University Press, 2002). 
learning phase of chunking bigger and bigger pieces together, I found it useful to rewrite the poetry to a score that did not have the percussion accompaniment notation. In addition to speed of learning, a primary objective in this strategy was to keep the poetry as the focus of the piece and the musical accompaniment within a supportive, secondary role. In my perspective, the busy and clunky aesthetic of the original score unwittingly ill-effected the flow of my recitation while developing my interpretation. In contrast, the above line of text (Fig. 5.5), jarred with musical symbols, becomes open for true vocal analysis and exploration when rewritten:

Let tul net us, use s nelt

Or, depending on your interpretation:

Lettul net us, use s nelt

Or the most fluid and generous to form combinatory phonemes:

Lettul netus, use snelt

I also applied this type of phonetic analysis to my interpretation of the B section, in which Smith uses conventional English words and syntactic devices with little adherence to small-scale, local meaning as it unfolds. In this way, the basic aural principles of poetry should be applied in order to convincingly portray Smith's "dream-like" musicality. As a piece of poetry, a realization of Tunnels must consider the natural stresses and durations of each line, which will help identify the musical components such as notes and rests, tempo, accent, meter, and rhythm. Smith does not provide duration or other timing element details in the score, admittingly granting the performer such flexibility in their realization:

"The visual arrangement of the score communicates formal/conceptual meaning, not implied timings. Letters, phonemes, or words should flow together as if you are saying makes absolute conventional linguistic sense."

Therefore, the performer's interpretation of durations and pauses should maintain conventional syntax, despite the lack of local meaning within the English text. The rhythmic 
elements must also work in conjunction with the composer's explicit timing instructions, which include the period symbol, indicating "ca 2 seconds" of instrumental accompaniment or "ca 2 seconds" of silence, depending on what prefaces the symbol, as well as that a full performance should last between six and eight minutes.

Throughout his introduction to poetry, Pinsky avoids discussing meaning in the poems he uses as examples, focusing instead on the poets' complex manipulation of structural elements, such as rhythm, meter, form, and syntax, to create an organic aural aesthetic. This aural perspective parallels Smith's description of Tunnels in the program notes:

"I once had a dream when I was very little which never left me. In this dream, when people talked, they sounded like music instruments - this one a tuba - this one a violin - this one a snare drum, and so on. No words came out, just instrumental sounds. I was very disappointed when I woke up to find it was just a dream.

In my speech songs, I reverse the situation. Words come out, but it sounds like music. For me, this music-sense makes perfect sense."146

The overall rhythm of the B section is affected by various poetic devices manipulated by Smith. I analyzed individual words to discover natural stresses, but ultimately the accent and durations of the syllables are informed by their relationship with those around it. For example, even though the single word "no" might be accented when appearing by itself as an exclamation, its treatment changes within relation to the entire line (line 179):

no deeper than a heavy dew.

When the full line is said out loud without an artificial pretext, elements of its natural rhythm emerge. The line consists of four pairs of syllables in which the second syllable of each pair has more accent than the first. Therefore, "no" in this context is relatively unaccented. The duration of the first syllable of "deeper" is also significantly longer than the other syllables.

${ }^{146}$ Stuart Saunders Smith, Tunnels (Sharon, VT: Smith Publications, 1988), program notes. 
Furthermore, the slight pause occurs after the word "deeper," therefore "than a heavy dew" seems to roll along quite fast in relation to the line's first three syllables.

Smith's use of punctuation also contributes to the rhythm of a poetic line. There are four instances where punctuation is used in the middle of a line: after "sing" (line 61), "alone" (line 66), "tongue" (line 137) and "yes" (line 176), which can affect the release of the last syllable prior as well as incorporate a pause. An example comes in line 66:

\section{Alone, the inverted Cone}

Without the comma, "alone" might be read as a weak beat followed by a stressed beat:

\section{Alone the inverted Cone}

Yet with the comma in place as written, the reader discovers that the feet exist in the reverse. The stress occurs at the onset of the line, and the second syllable of "alone" has an extended duration in addition to its pronounced accent. A definitive pause in sound occurs at the comma. When recited naturally, what surfaces is an odd numbered (7) line whose punctuation significantly affects the accent, duration, and meter.

The performer of Tunnels must also consider the formal aspects of line and syntax.

According to Pinsky:

"one of the most important principles...the line and the syntactical unit are not necessarily the same. Much unsatisfying reading and much inferior writing proceeds from not getting this idea right." 147

Pinsky describes how the rhythmic unit of a line should influence one's interpretation of an enjambment, the break of a line without punctuation or other break in syntax. For example, the syntax and written line may not correlate, especially in moments when the sentence simply would not fit onto a single line due to the restraints of paper. To borrow Pinksy's adjectives, the

\footnotetext{
${ }^{147}$ Robert Pinsky, The Sounds of Poetry: A Brief Guide (New York City: Farrar, Straus and Giroux, 1998), 30.
} 
rhythmic "dancing" of the text and the fluidity of the syntax suggest that lines 109 - 111 should be recited with great fluidity, despite the breaks in lines:

\section{The fourteenth letter plus twenty-nine}

and one half days equals its

mean density abbreviated $M$.

Finally, in order to sound natural and create an aesthetic beauty to the recitation, much like Pinsky's description of a poem's reading in the inner voice, lies in the realm of degree - not whether there are pauses between phonemes, combinatory phonemes, lines, and stanzas, but to what degree such a pause will be taken. Extremes of sound and rhythm can be incorporated for dramatic effect, but sensitivity must be applied to avoid sounding artificial.

Upon completing a satisfactory interpretation of the text, the percussionist should gravitate their attention to the musical accompaniment. Smith suggests "gradually add[ing] the instrumental accompaniments, unisons, and imitations of the words," but a consideration of the possible instruments, and the possible timbres able to be produced on those instruments, could be a distinguished phase. In terms of imitating the voice, specifically, the instrumentation should be constructed around an extended range of articulation, duration, and release. If the vocal interpretation does not include an extended pitch and dynamic range, then neither should one's instrumentation choices. For example, since the only words with full capitalization appear without musical accompaniment, "USELESS" (line 74), "WORLD" (line 79), and "MUTABLE" (line 145), I did not have to seek out an instrument that can produce a high dynamic. The only explicit exception of dynamic occurs in line 118 to accompany "brabble," which is subsequently performed without vocal presence in lines 120 - 124.

Instruments that have unique and overt pitch changes are invaluable for constructing close associations between vocal sound and musical accompaniment that can be perceived by the audience. Those amendable to perform glissandi or pitch bends are even more advantageous. 
Without a rich palette of pitches in the instrumentation, the musical accompaniment would be perceived as monotone, a stagnant pitch component viewed as a taboo way of speaking that the performer should avoid, or at least reserve for specific moments. Of course, approaching a question mark is typically executed by a rise in pitch, but there are other more subtle changes of pitch within word and sentence structure. Whether in isolation or in the context of the line and syntax (following the punctuation), words such as "metallic" (line 102) have an active pitch component. While I initially thought that I would naturally rise in dynamic when approaching an exclamation point, self-reflection and analysis illuminated that I only slightly raised in vocal pitch and did not change dynamic at all. Instruments such as timpani, vibraphone, and tabla (bayan/baya, specifically) are capable of such sound manipulation through their naturally long sustains. On the other hand, use of such instruments requires careful attention to orchestrate sensitive, rhythmic releases that may take advanced coordination techniques.

An advanced conceptual interpretation should consider the character, or characters, of the narration: who is presenting this material? Stark changes in vernacular and syntactic structure suggest a narrator that runs the gamut of emotions and perhaps is imitating other people or society. For example:

Paltry
Potty
Are simply idiomatic words,
Under brimless coverings,
Which set caps for picking at flaws.

The narrator presents single word sentences of increasing silliness and cultural taboo, culminating with "potty," the sound of which is imitated in the musical accompaniment and extended for one period ( 2 seconds). This is immediately followed by a quite exquisite and articulate statement that displays self- and cultural awareness: 
Are imply idiomatic words, Under brimless coverings, which set caps for picking up flaws.

The presence of questions within the text requires an interpretation. One may interpret that the narrator is simply asking him/herself these questions, in which case the actor must seek to establish a reflective, contemplative mood. There are instances where a question is answered, such as:

\section{Daystars?}

Yes, passageways.

The answered questions pose another justification for the depiction of multiple characters in the piece. Afterall, Smith has described the childhood dream that inspired the piece to have contained people that sounded like different musical instruments when they talked. The quick juxtaposition of text styles and proximity of the responses to the questions posed make integrating multiple characters in one's interpretation a precarious one. It may be that the performer choreographs changes of facial expression, vocal timbre, and pitch, because there simply may not be enough time to incorporate other characterizations such as staging or body postures. For the theatrical intricacies such as facial expressions and novelty of the small found instruments to be observed by the entire audience, I chose to perform this piece downstage center.

The performer must consider the conceptual facet of articulating form in Tunnels. As described previously, Welsh's overarching formal analysis via the text is straightforward and effective for the performer: non-lexical (A), standard English vocabulary (B), and non-lexical 
(A'). ${ }^{148}$ The vocal silences between each section further demarcate them. In an interview, the composer told Welsh:

"the reason the forms are simple is because they're not the point - the point is the detail. In order to make detail the point you have other things subservient. If you have very complex details and very complex forms, I think one will cancel out the other...In my music, I'm interested in very elaborate, elegant melodic writing. Form has always been a backdrop to that." 149

For those that prepare the work or analyze the score, the structural clarity is obvious. However, the advanced conceptual performer must consider the perspective of the audience member who is unfamiliar with the piece and only experiences it as it occurs in real time. ${ }^{150}$ The tertiary structure of the work could be articulated with change of lighting, costume, character, and instrument design, among others, as well as permutations of the aforementioned elements. If too many of these facets are chosen, the performer runs the risk of detracting from Smith's concept and even risks insulting the intelligence of the audience. For this reason, I chose to only articulate the formal constructs through musical means, choosing an instrumentation in two instrument groups: found instruments and standard percussion instruments. To avoid being too overt, I integrated the instrument groups together within a single setup design (Fig. 5.8), which imparted a small consequence of extending the overall setup design and made traversing the instruments in a local phrase or section more technically challenging.

\footnotetext{
${ }^{148}$ Welsh further analyzes the B section, exploring Smith's compositional processes for creating levels of meaning within the local incoherency by surveying various definitions and perspectives of the word "tunnels". Welsh, 164 173.

${ }^{149}$ Ibid.
} 


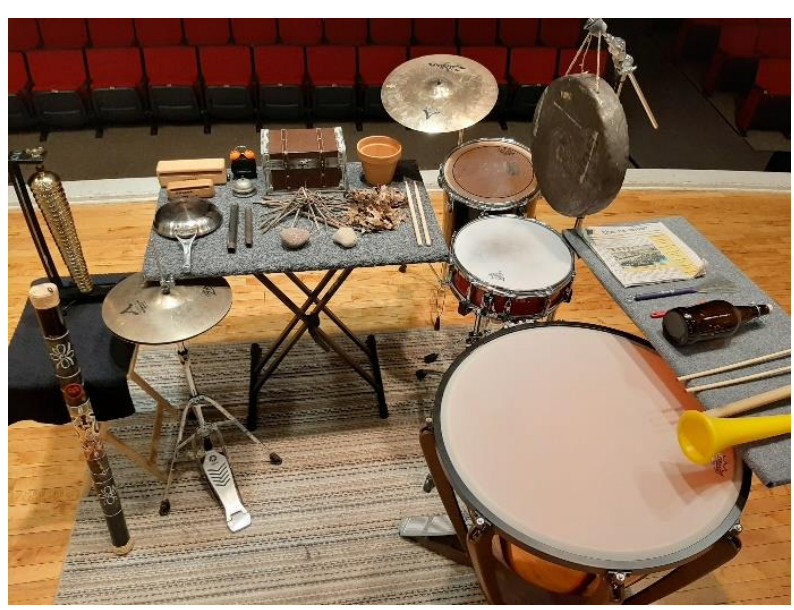

Fig. 5.8 Tunnels, Greco setup

For Sections A and A', I chose to restrict myself to found instruments from nature (rocks, wood, air, leaves, and other plant materials). I wished to make the association between the text's inner phoneme exploration of the word "tunnels" and the use of basic, earthly materials: those in which everything else is made. For the grace note instrumental noises, I orchestrated man-made found instruments (radio, frying pan, buzzer, bell). The transition to the B section continues the "tunnels" musical accompaniment for six seconds, which I orchestrated by digging, or "tunneling," into a storage box. I then pulled out a triangle beater and a maraca from the trap case, which I used to strike and scrape standard percussion instruments that accompany the beginning of the B section. This unexpected theatrical action serves to formally mark the poetic change, making a memorable association for the audience. The transition back to the A material was orchestrated with a rainstick that may be associated with the word "dew" (line 179). The rainstick also recalls the audience's memory of the previous transition through its association with the cylindrical shape of a "tunnel."

Tunnels is an advanced conceptual performance work for solo multiple percussion. The presence of isolated phonemes and non-lexical words that explore various sounds from the word tunnels present advanced problems such as diction. Likewise, the piece's exploration of the 
multiple meanings of "tunnels" presents associations that need to be addressed theatrically to communicate to the audience. The concepts are completely disparate to the other dimensions of multiple percussion: equipment and setup, standard notation, and technical demands. Yet, other conceptual performance aspects interrelate very closely with the other dimensions, such as an intelligent, varied instrumentation that can aid in the musicality of the vocal text and create extramusical associations, while also offering ample room for theatrical drama presented in the body and face that can be observed by the audience. 


\section{CHAPTER 6: CONCLUSIONS}

Several conclusions were made from the research conducted for this document. From a personal standpoint, this research led to a finer articulation of the various dimensions of the multiple percussion medium: (1) equipment and setup, (2) notational style, (3) technical demands, and (4) conceptual performance. Certain facets within these dimensions reappeared throughout the intermediate to advanced solo repertoire, suggesting that their fluency is requisite for the multiple percussion specialist.

I have concluded that there are both points of independence and interdependence between the dimensions and facets of the solo multiple percussion medium. This is not a novel discovery; the interrelationship between the size of a piece's instrumentation and the relative technical difficulty of traversing that setup is well documented. Further points of interrelationships were illuminated in this research, such as how the size of instruments and overall instrumentation also influence how the performer considers the audience sightlines, gauges the size of gestures, and chooses instruments for theatrical effect in their conceptual interpretation. Many other analogies and relationships are described within this document and are used in a general sense to create effective curricula, but a truly thorough analysis and description proved to be beyond this document. I hope instructors feel encouraged to seek these interrelationships in their available literature.

I can also conclude that each dimension progresses from explicit directions to implicit instructions and ultimately to the performer acting as part-composer. These require a clear continuum in terms of Bloom's taxonomies, the complexity of tasks. Therefore, a strategy in any instructor's curriculum may be an attentive, sensitive negotiation away from explicit directions on the score to favor implicit information that is illuminated via engagement with the piece. The 
implicit, experiential learning then might pave the way to opportunities where the performer has a heightened role as part-composer. This explicit-implicit-composer continuum was found to preside in all four dimensions of multiple percussion. In the equipment and setup dimension, explicitly prescribed instrumentations and setup diagrams in the intermediate repertoire lead to more complex tasks of creating one's own setup diagram, choosing instruments, and even constructing their own instruments and mounting solutions. In the notational realm, more concrete information is provided with standard Western conventions, while graphic and spatial notational devices might require detailed analysis for implicit suggestions and, in the most complex cases, compositional processes to realize. Prescribed techniques such as stickings and roll speeds are not given in the flagship advanced literature; those composers choosing to emphasize gestures, indeterminate rhythms, and other aspects that require the performer to problem solve and execute their chosen techniques. Similarly, certain works in the theatrical percussion realm are great avenues to introduce the facets of concept performance because the composer explicitly directs the performer. However, in congruence with Mason, I believe that a more interesting and individual portrayal of any work is produced when theatrical and dramatic elements are applied. ${ }^{151}$ Therefore, an expert multiple percussionist must apply theatrical elements to pieces even when no explicit theatrical instruction is given, complex tasks to formulate appropriate origination. These are just some examples of how the explicit-implicitcomposer continuum is present in each dimension, in which I hope a future researcher performs a more thorough study

Given the literature available for me to conduct this research, I propose the following graduate curriculum for a percussionist wishing to specialize in multiple percussion (Fig. 6.1 and

${ }^{151}$ Mason, "The Synthesis of Artistic Elements in Works for Theatrical Percussion," 4. 
Fig. 6.2). Of course, works for percussion ensemble, mixed chamber, and large ensembles can supplement this curriculum. However, within the solo realm, the percussion instructor is able to provide a more hands-on approach that will ensure a comprehensive curriculum that will develop the well-trained multiple percussionist. Common to typical graduate programs and the perspectives of instructors, the first semester of each program, master's and doctoral, has been dedicated in part to etudes and other works that ensure basic principles are understood, with potential deficiencies addressed.

\begin{tabular}{|l|l|}
\hline \multicolumn{1}{|c|}{ First semester } & \multicolumn{1}{c|}{ Second semester } \\
\begin{tabular}{|l|l|} 
Fundamentals via Udow/Watts and Petrella \\
conceptual performance
\end{tabular} & $\begin{array}{l}\text { Joe Tompkins, To Varèse } \\
\text { equipment and setup } \\
\text { technical demands } \\
\text { Michio Kitazume, Side by Side } \\
\text { notational style } \\
\text { technical demands } \\
\text { Thomas Svoboda, Discernment of Time } \\
\text { conceptual performance }\end{array}$ \\
\hline \multicolumn{1}{|c|}{ Third semester } & \multicolumn{1}{c|}{ Fourth semester } \\
$\begin{array}{l}\text { John Cage, Child of Tree } \\
\text { notational style } \\
\text { conceptual performance }\end{array}$ & $\begin{array}{l}\text { Dave Hollinden, Cold Pressed } \\
\text { equipment and setup } \\
\text { Benjamin Finley, Blade } \\
\text { equipment and setup } \\
\text { technical demands }\end{array}$ \\
& $\begin{array}{l}\text { Iannis Xenakis, Rebonds A/B } \\
\text { technical demands } \\
\text { William Hibbard, Parson's Piece } \\
\text { notational style } \\
\text { conceptual performance }\end{array}$ \\
\hline
\end{tabular}

Fig. 6.1 Sample Master's Degree Program Curriculum 


\begin{tabular}{|c|c|}
\hline $\begin{array}{l}\text { First semester } \\
\text { Stuart Saunders Smith, Songs I - IX } \\
\quad \text { equipment and setup } \\
\text { notational style } \\
\text { conceptual performance } \\
\text { Supplement fundamentals with Udow/Watts and } \\
\text { Petrella }\end{array}$ & $\begin{array}{l}\text { Second semester } \\
\text { Vinko Globokar, Toucher } \\
\text { conceptual performance } \\
\text { David Lang, The Anvil Chorus } \\
\text { equipment and setup } \\
\text { technical demands }\end{array}$ \\
\hline $\begin{array}{l}\text { Third semester } \\
\text { Morton Feldman, The King of Denmark } \\
\quad \text { equipment and setup } \\
\text { notational style } \\
\text { Kevin Volans, She Who Sleeps with a Small } \\
\text { Blanket } \\
\quad \text { technical demands }\end{array}$ & $\begin{array}{l}\text { Fourth semester } \\
\text { Vinko Globokar, ?Corporel } \\
\text { conceptual performance } \\
\text { Iannis Xenakis, Psappha } \\
\text { equipment setup } \\
\text { notational style } \\
\text { technical demands }\end{array}$ \\
\hline $\begin{array}{l}\text { Fifth semester } \\
\text { Karlheinz Stockhausen, Zyklus } \\
\text { equipment and setup } \\
\text { notational style } \\
\text { technical demands } \\
\text { conceptual performance } \\
\text { Stuart Saunders Smith, Tunnels } \\
\text { equipment and setup } \\
\text { notational style } \\
\text { conceptual performance }\end{array}$ & $\begin{array}{l}\text { Sixth semester } \\
\text { Herbert Brün, Touch and Go } \\
\text { equipment and setup } \\
\text { notational style } \\
\text { conceptual performance } \\
\text { Michael Gordon, } X Y \\
\text { technical demands }\end{array}$ \\
\hline
\end{tabular}

Fig. 6.2 Sample Doctoral Degree Program Curriculum

All four dimensions are emphasized in every two-semester span, ensuring the student develops all the necessary skills on a gradual, consistent basis. Careful attention was also made that the student does not have more than one piece with large instrumentation, thereby avoiding hindered practice time and other logistic frustrations. This suggestion is only to serve as an example for the concepts discussed in this document; it is my hope that the research articulates the dimensions of the performance practice and presents a model that will aid the instructor to 
construct their own curriculum, using their available library and incorporating future contributions to the literature.

The appendices that follow present my curricula specifically geared toward each dimension. The isolation and exploration of each dimension in the multifaceted model makes for a clear continuum of pieces that subsequently builds upon students' knowledge base and skill set, while posing further performance problems for learning. These curricula emphasize the relative independence within each dimension by isolating certain performance problems. In doing so, the instructor can create appropriate program goals and student outcomes individual to the student by choosing pieces to develop deficiencies and inexperience, or even to play to their strengths.

I found myself frustrated that certain facets of the performance practice avoided objective analysis or simply did not fit well into my proposed dimensions, so they were not added to the document. James Tenney's Having Never Written a Note for Percussion is an exemplar piece that poses challenges difficult to evaluate, primarily endurance and focus in terms of listening with intent. I decided that duration of a typical performance or the number of measures was an inappropriate way to evaluate endurance and focus and eventually resigned to exclude them from the study.

This project was also limited in terms of its scope. For one, it was restricted to solo repertoire in the acoustic realm, while a future study should expand this to include solo literature with electronic components or even multiple percussion with the chamber ensemble setting. In this case, I really look forward to reading future research perspectives into musical balance and sensitivity, how those concepts play into the multiple percussion medium, and how they may be taught in an academic setting. Theories and techniques of teaching performance practice in academia may change, as may ideas about appropriate graduate study pedagogy and scope. 
I hope that this document inspires future researchers; there are several ways in which I currently envision that this study could be expanded, some of which were already described in this chapter. In general, my committee and I certainly recognized the ambition of this project at the proposal stage. In the end, the document introduces the different dimensions that make up this multifaceted performance practice. I hope that this document sets the stage and encourages future researchers to delve into further research and to detail each dimension even more, especially in an effort to advance pedagogical practices. 


\section{APPENDIX A: EQUIPMENT AND SETUP CURRICULUM}

Charles DeLancey, The Love of L'Histoire (Los Angeles: Mitchell Peters, 1973)

Instrumentation: snare drum, bass drum, suspended cymbal, sizzle cymbal, tambourine (mounted), temple block, three woodblocks, cowbell

DeLancey's work is the quintessential intermediate piece in terms of equipment and setup. While the instrumentation as a collective is rather extensive, each instrument is standard for intermediate percussionists and percussion studio inventories. The provided setup diagram gives mounting and tuning information, even placement of the music stand. Attention will be needed to set up the woodblocks and cowbell in such a way that is resonant and easy to play. Tambourine is mounted on a cymbal stand, an extended technique quite standard today.

Other notable challenges: notation (intermediate), technical (intermediate), conceptual (intermediate)

Dave Hollinden, Cold Pressed (Greensboro, NC: C. Alan Publications, 1994)

Instrumentation: snare drum, two toms, foot pedal bass drum, bongos, tambourine, three cowbells, two woodblocks, two temple blocks, two crotales, splash cymbal, crash cymbal, ride cymbal

The piece calls for relatively standard percussion equipment for percussion studios. However, with an instrumentation that is quite wide and varied, performers may incur difficulties in mounting and placements. A detailed pictographic setup diagram is provided, as well as information about the timbre-staff notation and setup. Details of the drum tunings and manipulations of resonance are also given. Managing placement is a challenge and is ultimately a great exercise in creating a succinct setup that allows for unimpeded maneuvers without getting caught hitting other instruments. The mounting of two individual crotales may present a problem. The marriage of setup and notation in the timbre staff makes Cold Pressed an advantageous piece in any curriculum.

Other notable challenges: notation (intermediate-advanced) and technical (intermediateadvanced)

Joe Tompkins, To Varèse (New York: Bachovich Music Publications, 2013)

Instrumentation: concert bass drum (foot pedal), cabasa (foot pedal), tambourine (foot pedal), tamborin, snare drum, floor tom, siren (optional)

Other notable equipment: three foot pedals, timpani stool

To Varèse is an excellent case study in specific problems posed in the equipment and setup dimension. Specific dimensions are given by the composer, such as 28 " concert bass drum and a 16" floor tom; available equipment should be manipulated if exact instrumentation can be obtained. The tamborin (tamborim) is the only unusual instrument for a typical percussion studio, but it is the mounting of the instruments that pose the biggest issue. Students will either 
have to invest in the commercially available hardware, or they will have to manufacture their own creative solutions.

Other notable challenges: technical (intermediate-advanced)

Rickey Tagawa, Inspirations Diabolique (Greeley, CO: Western International Music, Inc., 1965)

Instrumentation: two sets of high and low suspended cymbals, bass drum, field drum, low and high snare drums, bongos, tambourine

Other notable equipment: implements (triangle beater, wound mallets, snare sticks, brushes, rattan)

Inspirations Diabolique contains standard percussion equipment and a relatively small instrumentation. In addition, a setup diagram is provided in the score. However, there are no instructions provided toward the mounting of the instruments and few concerning sound properties of the instruments. These qualities are in fact paramount in the performance of the piece, given the open musical texture and the sounding of instruments in isolation. The piece also calls for a wide variety of implements. Playing spots are specified in the piece, so it is important to choose instruments that create all the desired sounds, ultimately making for a detailed instrument choosing process. The significance in the equipment and setup dimension without explicit directions provided make this a pivotal work for the intermediate-advanced percussionist.

Other challenges: notation (intermediate), conceptual (intermediate)

Rolf Wallin, Stonewave (London: Chester Music Ltd. 2001)

Equipment needed: ten metal instruments, one large tam (foot pedal), five high-pitched wood instruments, five tom-toms, concert bass drum, five high-pitched wood or metal instruments, five skin drums (congas or bongos), bass drum (foot pedal)

The instrumentation of Stonewave (1992) makes the piece an absolute tour-de-force. The space needed for the thirty-three instruments is monumental, but the provided setup diagram does speed up the learning process. Since the piece is divided into two instrument stations that sound separately in the piece, one might practice each station in isolation to alleviate stress on the percussion studio. The piece can serve as an introduction to indeterminate instrumentation, as select instrument groups are chosen by material. Given the fast passages in the piece, the primary consideration should be for instruments that are sturdy and have a wide, flat playing surface. Placement of instruments is key to minimize the span of this huge setup. The large metal instrument, which the composer suggests should be a bass steel drum or large tam-tam, poses mounting and sound property problems.

Other notable challenges: technical (advanced) 
Maki Ishii, Thirteen Drums (Munich: Rob. Forberg Musikverlag, 1986)

\section{Instrumentation: twelve drums, bass drum (foot pedal)}

The initial challenge is likely acquiring the number of drums, the necessary hardware, and the space needed to execute the piece. Yet, further considerations into the technical demands, as well as negotiating the indeterminate rhythms, illuminates more difficult problems of setup design. Since virtuosic skills are needed to quickly maneuver the surfaces, care must be taken to choose drums with smaller head sizes that can be mounted very close to one another. This is counter to the composer's suggested instruments or a general desire to use Afro-Cuban or (bongos and congas) Japanese (taiko) drums for dramatic effect, all of which require special mounting considerations and large amounts of space. There are three basic models for one's setup design: chromatic, pyramid, and proximity-speed. ${ }^{152}$ Each model has resounding implications for the necessary techniques of execution and the audience's perception.

Other notable challenges: technical (advanced)

Karlheinz Stockhausen, Zyklus (London: Universal Edition Ltd., 1960)

Instrumentation: marimba, vibraphone, four tom-toms, snare drum, guiro (mounted), two African log drums, suspended cymbals, hi-hat, four almglocken, suspended bells (Indian or substitute mounted tambourine), two triangles, gong, tam-tam

Other notable equipment: exhaustive variety of implements

The required space needed to accommodate the vast number of instruments and hardware needed for the piece makes Zyklus (1959) a monumental undertaking. Since most of the instrumentation is standard equipment that will need to be used by other students and ensembles in the percussion studio, the performer needs to be adept in their set-up and tear-down processes. Given the advanced technical facility required to navigate around the setup, attention to detail is necessary for the placement of every single instrument. With such a heterogenous instrumentation, excellent mounting techniques and implement choices are crucial for every instrument to speak. The mounted guiro and triangle pose particular problems of mounting.

Other notable challenges: notation (advanced), technical (advanced), conceptual (advanced)

Stuart Saunders Smith, Tunnels (Sharon, VT: Smith Publications, 1988)

Instrumentation: indeterminate

Stuart Saunders Smith's Tunnels is an advanced equipment and setup task within the indeterminate instrumentation repertoire. There are many detailed facets in the brief that need to be met by a chosen instrumentation. Consequently, instrument and implement choices, as well as varied performance techniques, should be wide enough to imitate all the linguistic sounds and meanings of the text. The recitation of the text should inform much of these choices, a unique task for multiple percussionists. Choices should be made with precision and care so that the

\footnotetext{
152 Berry.
} 
instrumental music marries the text. Few resources to aid in decisions are available for the performer, both in terms of composer suggestions and recordings. The conceived dramatics of the theatric piece may influence instrument choice for dramatic effect (size, color) as well.

Other notable challenges: notation (intermediate-advanced), conceptual (advanced)

David Lang, The Anvil Chorus (New York: Red Poppy, 1991)

Equipment needed: three resonant metal instruments, four semi-resonant metal instruments, four non-resonant foot pedal instruments, two woodblocks, bass drum (foot pedal).

Other notable equipment: five foot pedals

All facets of the equipment and setup dimension pose problems that require creative solutions in The Anvil Chorus: instrument choice, mounting, placement, and setup design. Interpretive skills are needed to determine the place of pitch, tonality, and resonance within each metal group. Attention should be paid to the relationship between the different instrument groups, choosing either a unified, modular, or atomized model. ${ }^{153}$ Non-traditional, found instruments are encouraged to imitate the metal-working, unrefined character of the piece. Full analysis of the piece is necessary to create a cohesive setup design appropriate for the entire work. Implements should be chosen carefully to create a balance in volume between all instruments, likely requiring implement changes.

Other notable challenges: technical (advanced)

Iannis Xenakis, Psappha (Paris: Éditions Salabert, 1976)

Instrumentation: indeterminate in groups: three groups of wood/skin (three instruments each) and three groups of metal (two groups of three instruments and one group of one instrument)

Like The Anvil Chorus, Psappha (1975) poses problems via the construction of indeterminate instrument groups, but the issue is furthered by Xenakis' scoring for three different materials: wood, skin and metal. Navigating between wood and skins with the same instrument groups (Groups A, B, and C) poses a specific problem in terms of intra- and inter-group unity. Explicit instructions are virtually absent in the work, and implicit information only surfaces through analysis and firsthand experience with the score. While there is not one single school of thought concerning the instrumentation or setup, the piece is very well-known by experts; thereby, the evaluation of a performer's realization is likely inevitable.

Other notable challenges: notation (advanced), technical (advanced)

\footnotetext{
${ }^{153}$ Schick.
} 


\section{APPENDIX B: NOTATION CURRICULUM}

Charles DeLancey, The Love of L'Histoire (Los Angeles: Mitchell Peters, 1973)

Notation style: standard rhythmic notation on a grand staff (two five-line staves)

The score The Love of L'Histoire contains many conventional devices and typical percussion symbols, making it an accessible choice within the percussion curricula. Standard rhythmic notation is used throughout, with notes ranging from thirty-second to quarter notes. There is conventional treatment of meter, although meter changes might pose a reading problem for some. DeLancey incorporates several helpful aids for the reader, such as hiding staves with no material on a line, adding a single added line (tambourine), and using unique noteheads and pictogram symbols to help isolate members of the metal and wood groups. Rhythms are not beamed across the grand staff, making some deciphering necessary to discover the composite rhythms when both staves are sounding.

Other notable challenges: equipment and setup (intermediate), technical (intermediate), conceptual (intermediate)

William Ortiz, Tamboleo (Lancaster, NY: AM Percussion Publications, 1986)

Notation style: standard rhythmic notation on line score (two lines)

Ortiz's work is a great initial work in line score, while still appropriate for recitals. Besides the brief vocal introduction that uses the treble clef and a five-line stave, the entire piece maintains a line score that brackets two separate lines. Four instruments are scored, notated in the space either below or above its corresponding line. The rhythmic material between the two lines are not beamed together, making some passages difficult to determine composite rhythms. Clear dynamics are given throughout the piece, with intuitive placement for voicings and balance and even courtesy markings for clarity. Occasional measure numbers aid in teaching scenarios.

Other challenges: technical (intermediate)

John Cage, Child of Tree (New York: C. F. Peters Corp., 1975)

Notation style: entirely written prose description

Child of Tree is an aleatoric work for ten natural, often plant-like, instruments. The score is strictly prose description as well as tables of numbers that model various chance scenarios. The handwritten instructions provided by Cage are extremely rough and difficult to interpret; words and sentences are difficult to read, many of which are crossed out with various scribblings scattered throughout the page. The unclear directions make the interpretation process analytic, reflective and ultimately personal. The sonic material of the performance is improvisatory, with only the structure determined moments prior.

Other notable challenges: conceptual (intermediate) 
Iannis Xenakis, Rebonds A/B (Paris: Éditions Salabert, 1991)

Notation style: standard rhythmic notation on five-line stave(s)

The notational dimension for Rebonds (1987 - 89) fits nicely into the intermediateadvanced curriculum utilizing the five-line stave. The relative accessibility of the notation makes the piece a great choice for students that are especially strong in their technical facility. Melodic treatment of a relatively homogenous set of instruments makes the notational style very intuitive. The fast rhythms (up to sixty-fourth notes) in the slow tempo presents a sharp learning curve, but fortunately the beaming is very courteous to the performer.

Other notable challenges: technical (advanced)

Michio Kitazume, Side by Side (Tokyo: Zen-On Music Co., Ltd., 2005)

Notation style: standard rhythmic notation (without meters or bar lines) on a grand staff (two five-line staves)

The key for Side by Side (1977) is very typical of intermediate-advanced solos in the repertoire. However, the extended use of bracketed patterns that are to be repeated a specific amount of times makes this work a notational challenge. These repeated cells have a great range of duration from the shortest (one-note) cell to longest (fifty-one notes). The cell concept transfers to chamber ensemble pieces such as Terry Riley's In C (1964), Lukas Foss's Paradigm (1968), and several pieces in Steve Reich's catalogue.

Other notable challenges: technical (intermediate-advanced)

Dave Hollinden, Cold Pressed (Greensboro, NC: C. Alan Publications, 1994)

Notation style: timbre-staff

Hollinden's Cold Pressed (1990/94) is a piece that presents various challenges that should be attainable for a typical upperclassman percussionist. The timbre-staff notation presents an easily accessible relationship with the equipment and setup design, making a connection to other musical studies via the chromatic keyboard layout. Passages are rhythmically challenging, but the chromatic movement of the orchestration and general repetition of phrases make its reading relatively intuitive. Hollinden chooses to consistently use standard symbols for dynamic, tempo, meter, phrasing, and articulation. Descriptive terms for each section may present the only small conceptual challenge.

Other notable challenges: notation (intermediate-advanced) and technical (intermediateadvanced)

William Hibbard, Parson's Piece (Boston: E. C. Schirmer Music Co., 1976)

Notation style: extended rhythmic notation on line score (five lines)

The uniqueness of Hibbard's Parson's Piece (1968) presents further demands in line score reading and interpretation. The top line is the "attack rhythm," which visualizes the rhythmic composite of the music onto a single line. Not only is this an interesting idea, 
conceptually, but it also makes for a steep learning curve for the student percussionist, who has not likely seen such a notational device. It is possible to use the "attack rhythm" in all phases of piece's realization: learning, finalization, and either memorization (if one chooses) or reading during performance. The lower four lines notate the orchestration of the piece's twelve instruments, but no rhythmic information is provided on those lines, other than unison strikes that are obviously depicted directly vertical to one another. Groups of three instruments occupy each of the four lines: one on the space below, one on the line itself, and one the space above. The dynamics, articulations, and phrase markings are communicated via standard symbols on the lower four lines. The unconventional manipulations of standard notational devices place this piece keenly in the intermediate-advanced notation dimension.

Other notable challenges: conceptual (intermediate-advanced)

William Cahn, Nara (Everett, PA: HoneyRock, 1976)

Notation style: standard rhythmic notation on a grand staff (three five-line staves)

Nara is an extensive piece that proves to be a challenge in reading and interpretation. The piece extends many of the score reading challenges that present themselves in Tagawa's Inspirations Diabolique, such as written instructions and note head recognition. Despite the standard rhythmic notation used throughout, the three five-line staves are especially difficult to navigate when each are present. Rhythms are not beamed between the staves and, given its many large meters (such as 8/4), deciphering the composite rhythms and rhythmic relationships between the staves is quite difficult. Extensive performance notes must be internalized and kept in mind within one's realization process. Likewise, a plethora of detailed performance instructions are provided in the score itself, which are difficult to read while performing and perhaps require the performer to rewrite their own version of the score or memorize it. Provided the level of interpretation needed to realize this score, Nara is an appropriate intermediary into graphic and spatial scores.

Other notable challenges: equipment and setup (intermediate-advanced), conceptual (intermediate-advanced)

Stuart Saunders Smith, Tunnels (Sharon, VT: Smith Publications, 1988)

Notation style: text score

Tunnels is a unique score that integrates a variety of graphic symbols into written poetry. The text incorporates isolated phonemes, non-lexical gibberish, and nonlinear, ungrammatical English phrases that can be difficult to decipher. Three full pages of performance notes define the six graphic symbols that appear in the poetry. The piece may serve as an introduction to implicit directions and performer's compositional responsibility, because while the performer is free to develop their own realization of the musical material, one's recitation of the text, if not the text itself, provides sufficient implicit directions toward the music's rhythm and orchestration.

Other notable challenges: equipment and setup (advanced), conceptual (advanced) 
Stuart Saunders Smith, Songs I - IX (Sharon, VT: Smith Publications, 1981)

Notation style: hybrid of five-line stave, line score, and prose description

The varied notational devices make Songs I-IX an undertaking for the intermediateadvanced percussionist, perhaps a milestone before venturing into works with graphic and spatial notation. Movements combine different forms of notation, requiring the percussionist to synthesize different forms of information into one realization. Dramatic and theatric information is provided with long prose descriptions that leave room for interpretation as well.

Other notable challenges: conceptual (advanced)

Iannis Xenakis, Psappha (Paris: Éditions Salabert, 1976)

Notation style: graphic

The reading and interpretation of Psappha's score is certainly a daunting task. Despite a steep learning curve, however, the grid-like notational device is actually quite intuitive and legible. If the student's equipment/setup skills and technical facility are superior, the piece actually works quite well as one's initial piece in graphic notation. Implicit directions instruct on the indeterminate instrument, but outside of the equipment and setup dimension, much of the piece is explicitly given in the physical score.

Other notable challenges: notation (advanced), technical (advanced)

Morton Feldman, The King of Denmark (Glendale, NY: C. F. Peters Corp., 1965)

Notation style: graphic

The interpretation of The King of Denmark (1964) score requires attention to the explicit and implicit instructions, as well as a sense of compositional responsibility. Similar to Psappha, there are no spatial parameters to decipher, as the score of The King of Denmark is a grid that clearly defines the time axis; each box moving along the $x$-axis is a definite duration, between 66 and $92 \mathrm{bpm}$. Yet, Feldman only directs how many sounds occur in each unit of time, the sequence of events (rhythms) that occur is the responsibility of the performer. The $y$-axis is also clearly defined in terms of the relative register of the instruments into three categories (low, middle, and high), but many other facets of the orchestration are left to the performer. No instrument list or suggested setup is provided, making for a steep learning curve for this piece: it is likely that the percussionist will endure a long developmental process in creating their interpretation.

Karlheinz Stockhausen, Zyklus (London: Universal Edition Ltd., 1960)

Notation style: graphic and spatial

The score to Zyklus presents an advanced interpretative challenge for any percussionist. The English translation in the Universal Edition (1960) eliminates the linguistic challenge, but there is still plenty to interpret and synthesize within the performance notes alone. Six structures of the work, while clearly defined, are very complex and difficult to recall upon seeing their 
symbols on the score. Graphic symbols on the score itself are not necessarily intuitive. The sequence of sounds occurs spatially on the page with a single central system of time-lapses moving from left to right. Triangles extend from time-lapses; "zoomed-in" views of individual time-units. Inside the triangles are rhythmic cells whose sequence is chosen by the performer. Spatial factors also play a role; durations and time intervals of entries are drawn to scale, and dynamics are notated by the thickness of the symbol. Since the score can be read upside down or right-side up, there are many doubled symbols that occur on the left/right and top/bottom of the page, making for a cluttered score.

Other notable challenges: equipment and setup (advanced), technical (advanced), conceptual (advanced)

Herbert Brün, Touch and Go (Baltimore: Smith Publications, 1975)

Notation style: graphic and spatial

Touch and Go is a representative piece of the highest caliber in the notation dimension. Brün uses a variety of different graphic symbols to communicate very specific information, a big challenge being the recognition of thirteen different implements whose symbols are only marginally different from one another. Spatial devices are applied to each of the graphic symbols, with relative placement along the $y$-axis ("distance") indicating different factors depending on which symbol is being used. The performer must also negotiate implicit directions of instrumentation, as well as composing the sequence of sounds. The other two works that make up a trio by Brün, Plot and Stalks and Trees and Drops and Clouds, utilize similar computergenerated symbols, but within a different system of associations and parameters. Each of these works are notational juggernauts.

Other notable challenges: technical (intermediate-advanced), conceptual (advanced) 


\section{APPENDIX C: TECHNICAL DEMANDS CURRICULUM}

Charles DeLancey, The Love of L'Histoire (Los Angeles: Mitchell Peters, 1973)

The Love of L'Histoire is an all-encompassing piece for the intermediate percussionist. The piece emphasizes passages containing melodic movement between surfaces and one-hand independent ostinatos, while the other performs simple counterpoint. The creation of balance and melodic lines from an instrumentation of varied material should be emphasized. Tempo, meter, and character changes are prefaced with silence, allowing preparation for the change.

Other notable challenges: equipment and setup (intermediate), notation (intermediate), and conceptual (intermediate)

David Mancini, Latin Journey (Ashbury Park, NJ: Studio 4 Music, 1998)

Latin Journey has many similar technical demands as The Love of L'Histoire, but the style of the piece places more emphasis on hand independence, accent development, repetition and groove, albeit with a more homogenous instrumentation. Known Latin rhythms supply relatively complex ostinatos in one hand while other hands alternate between different divisions (i.e., half note, quarter note, dotted quarter note) of the groove. Consistency in rhythm and accent vs. unaccented development is necessary to bring this piece to life, a welcomed piece in the technical dimension curriculum.

Eckhard Kopetzki, Canned Heat (San Antonio: Southern Music Co., 2003)

Canned Heat (2002) is a next logical step for the development of technical facility in undergraduate students. The very few places of silence or open musical texture makes for an element of endurance in terms of "chops." The first section of the piece calls for one hand to hold two mallets, and the second section calls for some changes of implements, executed while one hand continues playing. The lengthy closing section is played with one drumstick in each hand. While there are a few tricky spots in terms of rhythmic reading, the primary challenge are the counterpoint and polyrhythmic figures throughout the piece and the occasional fast flourishes.

Michio Kitazume, Side by Side (Tokyo: Zen-On Music Co., Ltd., 2005)

Side by Side is a drumming piece that requires well-developed single strokes, hand independence, repetitious groove, and dynamic control. The piece is scored for six drums to be played with hard mallets and one foot pedal bass drum. Endurance is key, as the drumming is continuous throughout its repeated rhythmic cells. Foot pedal work is not excessive, mostly unison hits that require attention not to "flam" with other instruments. There are some tricky polyrhythmic passages, with the 5:4 polyrhythm reappearing throughout the work. The most complex polyrhythm contains a syncopated 4/4 ostinato in the right hand occurring over a dotted eighth note (i.e., 3/16 meter) in the left hand in which the bass drum marks every fifth strike (i.e., 15/16); the polyrhythm finally synchronizes after fifteen repetitions of the $4 / 4$ ostinato and sixteen repetitions of the $15 / 16$ pattern. 
Iannis Xenakis, Rebonds A/B (Paris: Éditions Salabert, 1991)

Rebonds is a virtuosic display of technical facility in two movements. Rebonds A builds from an open to an extremely thick texture that requires full facility around the seven drums. The rhythmic material is mostly simple divisions as fast as sixty-fourth notes and 4:3 polyrhythms. Rebonds $B$ contains manipulations of a syncopated pattern over a constant pulse in the high bongo voice, executed by a single stroke in one hand. These passages often alternate with sections of rolled notes, potentially executed with single-, double-, multiple-bounce strokes or a combination thereof. An advanced problem occurs from the orchestrated drags in the middle of the high bongo pulse, which requires mastery of motion, grip, and rebound control, regardless of the chosen sticking. The rolled notes in the woodblocks that occur underneath simultaneous counterpoint is a problem that requires creativity and technical prowess. Fast flourishes in the woodblock voices are extremely challenging, as well as the need to negotiate different amounts of rebound from the instruments in general.

Other notable challenges: equipment and setup (intermediate-advanced), notation (intermediate-advanced)

Kevin Volans, She Who Sleeps with a Small Blanket (London: Chester Music, 1996)

Volans created an extreme virtuosic technical display in his She Who Sleeps with a Small Blanket (1986). The majority of the piece contains fast flourishes across the six drums that require excellent control of movement over both $x$ - and $y$-axes. Like Side by Side, the bass drum simply accentuates attacks from the hands, presenting a more difficult technical task in a similar instrumentation and musical style. Change of rhythmic groupings occur throughout the piece, requiring fluency in all partials of the quintuplet. Relatively isolated sections bring special techniques such as double strokes and the holding of two-mallets in one hand. The closing section on marimba requires no special technical consideration, but does offer an opportunity to incorporate the keyboard into the multiple percussion curriculum.

Maki Ishii, Thirteen Drums (Munich: Rob. Forberg Musikverlag, 1986)

Thirteen Drums (1985) is a technical feat that would challenge any percussionist. The area that the setup covers will be great, regardless of chosen instruments or setup design. The percussionist must span the setup quickly as fast flourishes occur throughout the piece. Fast, onehanded single strokes that serve as the rhythmic accompaniment (á la Rebonds B) must be executed in both hands. Press strokes are also scored in the piece, but more serious problems come from negotiating the rolls; since they occur in many different contexts in the piece, they may be done with single-, double- multiple-bounce strokes or a combination thereof. The relatively short section that contains the use of hands/fingers and mallets pose relatively few problems. The performer must develop their gestures, especially in their treatment of the indeterminate grace-note fixtures.

David Lang, The Anvil Chorus (New York City: Red Poppy, 1991)

The Anvil Chorus presents numerous technical challenges for the percussionist. The feet must traverse five foot pedals: four of which are used to strike metal instruments and one for the 
bass drum. These instruments sound while both hands perform as well, requiring great motion, coordination and overall balance to execute complicated patterns. The second section poses advanced independence and coordination problems, as the woodblocks provide counterpoint over changing meters in the non-resonant metals, each playing intricate flourishes and patterns. The "much faster" section requires fast playing while maintaining a pianissimo dynamic, a particular challenge across seven abrasive metal instruments.

\section{Other notable challenges: equipment and setup (advanced)}

James Woods, Rogośānti (James Woods Ed., 1986)

Rogośānti requires a variety of percussion techniques to realize. The percussionist must be fluent in changing rhythmic groupings, which generally occur on a single line of material. Independence and coordination are emphasized as the melodic material is orchestrated across the piece's wide instrumentation. The piece necessitates a variety of strokes and interval control with four mallets. Rogośānti also requires the rhythmic use of vocals and further non-standard instrumentation, as well as quarter-tone tuning of glockenspiel bars.

\section{Other notable challenges: equipment and setup (advanced)}

Michael Gordon, XY (New York City: Red Poppy, 1998)

Technically, Gordon's $X Y(1997 / 2001)$ is an extremely advanced piece, despite being scored for just five tuned drums that are played with the same pair of mallets or sticks. $X Y$ contains fast drumming from beginning to end where both hands play rapid single strokes. Polyrhythms are explored from simple alternating sixteenth-notes (an offset 1:1) to the complex 6:5. Much of the piece contains crescendos in one hand, while the other has diminuendos, requiring absolute mastery of their independence and coordination. Patterns via orchestration also become more complex throughout the piece, developing into patterns that are measures long. Since the piece is marked at $202 \mathrm{bpm}$, which is all but impossible, performers must negotiate maximizing their tempo without sacrificing their accuracy or endurance.

Karlheinz Stockhausen, Zyklus (London: Universal Edition Ltd., 1960)

The performer is put to task for Zyklus, being completely surrounded by an extremely heterogenous instrumentation. The percussionist must utilize a variety of techniques from various stroke types, scraping of the guiro and keyboard glissandos, rolls on the triangle, and even foot work with the hi hat and vibraphone pedal. Dense textures mean that independence is key, there are many instances that would benefit from a one-handed roll to continue on one instrument, while the other hand either performs counterpoint or changes implements. Playing spots are crucial to produce the desired sounds in select instruments. Four-mallet usage is presumed for this piece, in which mallet type/hardness needs to stay in one's awareness.

Other notable challenges: equipment and setup (advanced), notation (advanced), conceptual (advanced) 


\section{APPENDIX D: CONCEPTUAL PERFORMANCE CURRICULUM}

Charles DeLancey, The Love of L'Histoire (Los Angeles: Mitchell Peters, 1973)

The The Love of L'Histoire incorporates a number of conceptual performance facets that can be developed by the intermediate percussionist. The piece not only encourages an engagement with Stravinsky's Histoire du Soldat, but score study of both the full theater and suite versions will help inform their solo realization. For example, while articulation and phrase markings are all but absent in DeLancey's work, the chamber ensemble scores provide tangible and accessible data. There are significant changes in character that emulate the different movements of Stravinsky's work. DeLancey informs and assists the percussionist with clear, definitive tempo markings that are preceded by a repeated ostinato or significant rest.

Other notable challenges: equipment and setup (intermediate), notation (intermediate), technical (intermediate)

Thomas Svoboda, Discernment of Time (Portland, OR: T. C. Stangland Co., 2003)

Svoboda's Discernment of Time (1983) for solo tam-tam is an appropriate piece for the student to focus on conceptual facets, because equipment and setup, technical, and even notational dimensions certainly pose few problems. The piece calls for precise playing spots on the tam-tam stand as well as the instrument, including the diameter of the tam-tam being divided into twelve segments, symbolizing a clock-face. The performer must negotiate their movement to imitate clock hands as well. Regardless of their position, sitting or standing either in front or behind the instrument, the performer is exposed due to the open texture, small setup, and the lack of movement in the tam-tam (or the stand) when struck.

\section{Other challenges: notation (intermediate-advanced)}

William Cahn, Nara (Everett, PA: HoneyRock, 1976)

Nara contains many moments of great drama; the open textures and resonant instrumentation provide opportunities for dramatics. Indeterminate rhythms such as accelerando and ritardando figures provide ample space for gestures. There are a variety of performance techniques called on (i.e. striking, scraping, crumbling), and implement changes should be fully explored to create the diverse ethereal soundscape. The piece has programmatic titles for each movement, as well as descriptive instructions for the sections, providing space for the performer to flex their interpretive skills.

Other notable challenges: equipment and setup (intermediate-advanced), notation (intermediate-advanced)

William Hibbard, Parson's Piece (Boston: E. C. Schirmer Music Co., 1976)

The large, resonate instruments and open textures of Parson's Piece put a dramatic portrayal at the center of a successful performance. The percussionist should navigate time and space of the setup to create long, fluid movements that can be viewed by the entire audience. 
There are plenty of open spaces in which the performer can consider their theatrics. Another problem occurs with stage placement and lighting to create an effective dynamic and visual experience.

\section{Other notable challenges: conceptual (intermediate-advanced)}

Frederic Rzewski, To the Earth (Brussels: Sound Pool Music, 1985)

Rzewski's often-performed work for four flowerpots and voice is an effective work to introduce vocals in the curriculum. Typical performance practice of the work situates the musician in a new position: sitting on the ground using a found implement such as chopsticks or knitting needles. Another unconventional aspect is the vocal text, a Homeric hymn that honors the Earth. From a technical standpoint, both the recitation and the musical accompaniment alone are relatively easy. Yet, negotiating the relationship between the vocals and musical accompaniment, as well as maintaining a natural, organic recitation, pose serious challenges.

Other notable challenges: equipment and setup (intermediate)

Stuart Saunders Smith, ...And Points North (Sharon, VT: Smith Publications, 1990)

...And Points North is a quintessential piece that contains equal treatment of movement, sound, text, and theater. Smith provides relatively explicit directions for theatrical elements of this three-movement opera, such as facial expressions, stage logistics, lighting, and even costume. The complexity of the rhythmic groupings and polyrhythms, typical of Smith's style, restrains the performer from focusing entirely on the theatrical elements. Navigating meaning in the work and the recitation of vocal text in the indigenous Passamaquoddy language can pose problems.

Other notable challenges: equipment and setup (intermediate-advanced), notation (intermediate-advanced)

Stuart Saunders Smith, Songs I - IX (Sharon, VT: Smith Publications, 1981)

Songs I-IX add further facets of conceptual performance of which a multiple percussionist should seek fluency. Directions are less explicit than others in Smith's catalogue, influencing the performer to analyze and diagnose theatrical elements that are only implied and generally vague. There is a heavy use of vocals in the piece with rapidly changing demeanor and character that spans a wide range of emotions. Most found instruments are household items that should be carefully chosen for their color and novelty, as well as their sound properties and required performance technique.

Other notable challenges: equipment and setup (intermediate-advanced), notation (intermediate-advanced)

Herbert Brün, Touch and Go (Baltimore: Smith Publications, 1975)

Upon developing a basic understanding of the notational system for Touch and Go (1967), the performer must determine what to do with the information on the page. An interpretation of why Brün chose the parameters that are explicitly instructed on and how they 
were displayed in that manner. The quadrangles used to represent sonic information requires the performer to compose at every moment of the piece, subsequently building on the micro-level to create sentences (i.e., phrases) and ultimately a meaningful performance. Touch and Go is a heady, conceptual work that requires great interpretation, compositional, and realization skills.

Other notable challenges: technical (intermediate-advanced), conceptual (advanced)

Georges Aperghis, Graffitis (Paris: Éditions Salabert, 1980)

Graffitis is a monumental percussion piece in the conceptual performance dimension. The instrumentation is completely open, but there are many implicit directions coming from the instrument's performance technique, sound qualities and its mounting, and placement opportunities. The entire piece is scored for four groups of instruments, each of which are to be played with the hands. The piece quickly juxtaposes between determinant and indeterminate passages, including fermata and accelerando figures quickly that require interpretation. The vocal part requires excellent articulation, speed, and even coordination due to passages where it provides counterpoint with the instrumental music. Relatively loose theatrical directions are provided above certain fermatas with the English translation being provided by the composer. Common to Aperghis's style, the performer also has moments where they must mimic playing without producing sound. The indeterminate instrumentation, virtually no description of meaning in the performance notes, and the composer's directions in the score to use various "objects" in theatrical ways, results in many possible individual interpretations.

Other notable challenges: equipment and setup (advanced), technical (intermediateadvanced)

Stuart Saunders Smith, Tunnels (Sharon, VT: Smith Publications, 1988)

Tunnels is an advanced piece in the conceptual dimension. In comparison to the other pieces by the composer, Tunnels contains less explicit directions on realization and fewer clues toward the piece's meaning. The performer must vocalize gibberish, as well as English text that is used to deliver both coherent and incoherent small-scale sentences. The poetry that is created is an abstract, nonlinear narrative that requires analysis for proper realization. The performer must choose an instrumentation that supports the sounds and meanings of the text. Rapidly changing demeanor implied in the text suggests stark changes of emotions and perhaps different characters. Determining how to mark the simple form should be made to offer a reprieve from the complexity and abstract nature of the piece.

Other notable challenges: equipment and setup (advanced), notation (intermediateadvanced)

Vinko Globokar, ?Corporel (New York City: C. F. Peters Corp., 1989)

?Corporel (1985) is a true display of theatrical mastery. A performer may feel completely bare; their body is the only instrument. Conventional performance practices are completely stripped, as the performer must command centerstage, appearing in minimal clothing and performing wild and unusual theatrics. Nonverbal vocalization commands most of the score, but 
there is one poetic moment that can be recited in the original French or translated into their vernacular language. While certain movements and gestures are explicitly directed up, there are moments when facial expressions and bodily movements are not instructed, in which case the performer must serve as part-composer.

Other notable challenges: notation (intermediate-advanced) 


\section{BIBLIOGRAPHY}

Adams, Daniel. "The Compositional Use of Timbre in Selected Solo Multiple Percussion Works." Interface: Journal of New Music Research 21, no. 2: 117 - 134.

. "The Drum Set as a Solo Multiple Percussion Performance Medium." NACWPI Journal 52, no. 3; pgs. 4 - 13.

. "Striking Implement and Surface Area Specification in Unaccompanied Multiple Percussion Solos: Its Compositional Significance.” DMA document: University of Illinois at Urbana-Champaign, 1985.

Applebaum, Terry. "A Comprehensive Performance Project in Percussion Literature with an Essay Comprised of Multi-Percussion Performance Problems as Found in Selected Contemporary Works, with Original Etudes Relevant to those Problems." DMA document: University of Iowa, 1978.

Arnold, Robert. "There's No Sound in My Head: Mark Applebaum's Metaphysics of Notation." 2010. Lateral Films, Documentary, 19:43. August 26, 2010. https://vimeo.com/14469188.

Bajzek, Dieter. Percussion: An Annotated Bibliography with Special Emphasis on Contemporary Notation and Performance. Metuchen, NJ: Scarecrow Press, 1988.

Banek, Reinhold, and Jon Scoville. Sound Designs. Berkeley, CA: Ten Speed Press, 1995.

Berry, Mark. "Thirteen Drums for Percussion Solo, op. 66: Interpreting in Concurrence with Make Ishii’s ‘Space-Time’ Concept.” Percussion Notes 47, no. 5 (2009): 50 - 59.

Bliss, Andrew M. (ed.). Multitudes. Nashville: Innovative Percussion, 2009.

Brindle, Reginald Smith. Contemporary Percussion. London: Oxford University Press, 1991.

Brown, Steven. "A Study in Multi-Percussion Setup Design.” Percussive Notes 21, no. 2 (January 1983): 35-37.

Bump, Michael. "Focus Day 2015: Time and Motion: The Percussive Gesture." Percussive Notes 53, no. 4 (September 2015): 4 - 9.

Campbell, James and Julie Hill. Music for Multi-Percussion: A World View. Van Nuys, CA: Alfred Publishing Co., 2008. 
Catford, J. C. A Practical Introduction to Phonetics, $2^{\text {nd }}$ ed. New York City: Oxford University Press, 2002.

Chapman, Evan. “The Anvil Chorus by David Lang (Multiple Percussion)” (video). December 11, 2012. Accessed February 15, 2020. https://www.youtube.com/watch?v=2yB6QMMmCw.

Charles, Benjamin. "Multi-percussion in the Undergraduate Percussion Curriculum.” DMA document: University of Miami, 2014.

Chenoweth, Vida. "Four-Mallet Technique.” Percussionist 1, no. 3 (1963): 5 - 6.

Chernoff, John Miller. African Rhythm and African Sensibility: Aesthetics and Social Action in African Music Idioms. Chicago: University of Chicago Press, 1979.

Cook, Gary. Teaching Percussion with DVD, $3^{\text {rd }}$ ed. Belmont, CA: Thomas Schirmer, 2006.

Coleman, Matthew. "Instrument Design in Selected Works for Solo Multiple Percussion.” DMA document: Arizona State University, 2012.

Davis. Christopher. "Theories of Rhythm and Meter and their Pedagogical Implications for NonPitched Percussion Music with an Analysis of William Kraft's French Suite.” DMA document: University of South Carolina, 2011.

Derr, Eric. "Contemporary Percussion in the Age of Information Overload: Three Pieces for Percussion and Tape.” DMA document: University of California, San Diego, 2014.

Fallows, David. “Tenuto.” Grove Music Online. 2001. Accessed June 23, 2019. http://www.oxfordmusiconline.com

Flint, Ellen Rennie. "An Investigation of Real Time as Evidenced by the Structural and Formal Multiplicities of Iannis Xenakis' 'Psappha'.” PhD diss.: University of Maryland, College Park, 1989.

Gerber, Stuart. "Karlheinz Stockhausen’s Solo Percussion Music: A Comprehensive Study." DMA document: University of Cincinnati, 2003.

Glassock, Lynn. "Four-Mallet Grips.” Percussionist 11 (1973): 2 - 11.

Goldenberg, Morris. Studies in Solo Percussion. New York City: Chappell, 1968. 
Gould, Michael. "Advanced Multiple Percussion Techniques: An Analysis with Musical Approaches to Performance Problems in the Music of David Hollinden." DMA document: University of Kentucky, 1999.

Grayson, John. Environments of Musical Sculpture You Can Build. Vancouver: Aesthetic Research Centre of Canada, 1976.

Hampton, Walter. "Rhythmic Consonance and Dissonance in Eckard Kopetzki's Works for Solo Percussion: Topf-Tanz and Canned Heat." DMA document: University of North Texas, 2014.

Hinkle, Lee. “Theatrical Music for Solo Percussion.” DMA document: University of Maryland, College Park, 2010.

Hopkin, Bart. Musical Instrument Design: Practical Information for Instrument Making. Tucson, AZ: See Sharp Press, 1996.

Julian-Jones, Rachel. "Notational Nomenclature for Multiple Percussion." Percussive Notes 46, No. 2 (April 2008): 18 - 27.

. "A Survey of Multiple Percussion Notation with an Emphasis on Timbre Staff Notation and Setup.” DMA document: University of Nevada, Las Vegas, 2005.

Kamstra, Darin. "Multiple-Percussion Notation: The Effectiveness of Three Types of Staff Notation on Sight-Reading Ability.” DMA document: University of Illinois at UrbanaChampaign, 2006.

Karkoschka, Erhard. Notation in New Music: A Critical Guide to Interpretation and Realization. New York City: Praeger, 1972.

Keown, Matt. "The Anvil Chorus, by David Lang” (video). Vic Firth. March 9, 2017. Accessed February 15, 2020. https://www.youtube.com/watch?v=_DUFzprscPk.

Klopfenstein, Reginald. "The Solo Concertos for Multiple Percussion and Orchestra by Donald Erb and Robert Suderburg: An Analysis of the Musical Materials and Performance Problems.” DM document: Indiana University, 1995.

Koetting, James. “Analysis and Notation of West African Drum Ensemble Music." In Selected Reports 1, no. 3, (1970): 115-146. 
Krueger, Meri Jo. "Principles for Percussion Writing in Music that Combines Multiple Percussion for a Solo Player with Another Solo Instrument.” DMA document: University of Texas at Austin, 1997.

Lambert, James. "Multiple Percussion Performance Problems as Illustrated in Five Different Works Composed by Stockhausen, Smith Brindle, Colgrass, Dahl, and Kraft Between 1959 and 1967.” DMA document: University of Oklahoma, 1983.

Lane, John and Terry Longshore. "Focus Day 2014 - Images of Sound: Innovations in Notation." Percussive Notes 52, No. 5 (September 2014): 8 - 14.

Larkin, Barry. “A Performance Analysis of Psappha.” Percussive Notes 30, no. 6 (August 1992): $64-68$.

Lewis, Kevin. "Herbert Brün’s 'Stalks and Trees and Drops and Clouds.'” Percussive Notes 44, no. 4 (August 2006): 48 - 53.

Lewis, Kevin, and Gustavo Aguilar. The Modern Percussion Revolution: Journeys of the Progressive Artist. New York City: Routledge, 2014.

Licata, Julie. "Physical Gesture, Spatialization, Form and Transformation in Watershed I/IV for Solo Percussion and Real-Time Computer Spatialization, by Roger Reynolds.” DMA document: University of North Texas, 2009.

Liu, Yi-Jan. “Temporality and Rhythmic Structure in 'Thirteen Drums' by Maki Ishii and 'Rebonds A' by Iannis Xenakis.” DMA docuemnt: University of North Texas, 2014.

Mason, Karlyn. "The Synthesis of Artistic Elements in Works for Theatrical Percussion.” DMA document.: University of Miami, 2014.

Muller, Jeremy. "Real-Time Chance Operations: A Technologically Modern Approach to John Cage's 27'10.554" for a Percussionist." Percussive Notes Online Research Edition 1 (2016): $24-31$.

Meyer, Bradley. "Six Japanese Gardens and Trois Rivieres: Delta: An Analysis of Kaija Saariaho’s Two Major Works for Solo Percussion and Electronics.” D.M.A. diss.: University of Kentucky, 2011. 
Nichols, Kevin. "Important Works for Solo Drum Set as a Multiple Percussion Instrument." DMA document: University of Iowa, 2012.

O’Neill, John. "Recent Trends in Percussion Notation.” Percussive Notes Research Edition, Percussionist 18 no. 1 (1980): 20 - 55.

Palter, Morris. "The Solidification of Performance Practice Issues in Solo Percussion Performance.” DMA document.: University of California, San Diego, 2005.

Petrella, Nick, and Jon Allemeier. The Multiple-Percussion Book: Concepts for a Musical Performance. New York City: Carl Fischer, 2000.

Pinsky, Robert. The Sounds of Poetry: A Brief Guide. New York City: Farrar, Straus and Giroux, 1998.

Read, Gardner. Pictographic Score Notation: A Compendium. Westport, CT: Greenwood Press, 1998.

Richlin, Laurie. Blueprint for Learning: Constructing College Courses to Facilitate, Assess, and Document Learning. Sterling, VA: Stylus Publishing, 2006.

Rockwell, Owen. “Psappha by Iannis Xenakis: Developing Multiple Percussion Literacy.” DMA document: University of Southern Mississippi, 2015.

“Rzewski, Frederic.” IMSLP, accessed June 22, 2019. https://imslp.org/wiki/Category: Rzewski,_Frederic.

Sallak, Bill “Informed Indeterminacy: Guidelines for Instrument Choice in Iannis Xenakis' 'Psappha."” Percussive Notes 40, no. 2 (April 2002): 55 - 59.

Sauer, Theresa. Notations 21. New York City: Mark Batty Publishing, 2009.

Schick, Steven. The Percussionist's Art: Same Bed, Different Dreams. Rochester, NY: University of Rochester Press, 2006.

Schutz, Michael. "The Mind of the Listener: Acoustics, Perception, and the Musical Experience: Do Longer Gestures Make Longer Notes.” Percussive Notes 47, no. 5 (November 2009): $22-28$. 
Schutz, Michael and Fiona Manning. "Effectively Using Affect Gestures: What Percussionists Need to Know About Movement and Perception.” Percussive Notes 51, no. 2 (March 2013): $26-31$.

Scoville, Jon. "Found Sound." Percussive Notes 23, no. 1 (October 1984): 43 - 44.

Sharp, Jonathan. "A Performance Guide to Glenn Kotche's Monkey Chant." PhD diss.: University of Kentucky, 2014.

Smith, Alyssa. "An Examination of Notation in Selected Repertoire for Multiple Percussion." DMA document: The Ohio State University, 2005.

Smith, Gary. "Instrument and Implement Selection and Setup, Performance Strategies, Structure, and Interpretation in Helmut Lachenmann's Interieur I." DMA document: University of California, Los Angeles, 2016.

Sofin, Elizabeth. "Text as Music, Music as Text: Stuart Saunders Smith's Works for Percussion and Spoken Word.” DMA document: University of Arizona, 2017.

Strom, Julie. "Theater Percussion: Developing a Twenty-first-century Genre Through the Connection of Visual, Dramatic, and Percussive Arts." DMA document: University of Northern Colorado, 2012.

Thierauf, Andrew. "Percussion and Max: A Collection of Short Works for Solo Percussion and Live Electronics.” DMA document: The University of Iowa, 2015.

Udow, Michael. "Visual Correspondence Between Notation Systems and Instrument Configurations." Percussive Notes 18, no. 2 (Winter 1981): 15 - 29.

Udow, Michael, and Chris Watts. The Contemporary Percussionist: 20 Multiple Percussion Recital Solos. Fort Lauderdale, FL: Meredith Music Publications, 1986.

Varèse, Edgard. Ionisation, in Modern School for Snare Drum. Ed. Morris Goldenberg and Anthony Cirone. Van Nuys, CA: Alfred Music, 1955/2002.

Voight, Tyson. "Hearing What You See: A Case for the Use of Ancillary Gesture in Individual Percussion Performance.” DMA document: University of Miami, 2016.

Wadley, Darin. “...And Points North by Stuart Saunders Smith: An Examination of Musical Influences and a Performer's Guide.” DMA document: University of Arizona, 1998. 
Welsh, John P. The Music of Stuart Saunders Smith. Westport, CT: Greenwood Press, 1995.

Welwood, Arthur. "Improvisation with Found Sounds.” Music Educators Journal 66, no. 5 (January 1980): $72-77$.

Wier, Alexander. "Performer and Electronic-Activated Acoustics: Three New Works for Solo Percussion and Live Electronics.” DMA document.: Arizona State University, 2015.

Wiggins, Tracey. “27’10.554” by John Cage and The King of Denmark by Morton Feldman and Their Influence Upon Thomas DeLio's as though.” DMA document: University of Hartford, 2009.

Woodmansee, Brett. “Alternative Analyses of Three Multiple Percussion Works.” D.M.A. diss.: North Dakota State University, 2013.

Zeltsman, Nancy. “Traditional Four-Mallet Grip.” Percussive Notes 33, no. 4 (1995): 50 - 54. 